\title{
Boosting Fmoc Solid-Phase Peptide Synthesis by
}

\section{Ultrasonication}

Francesco Merlino, ${ }^{a} \ddagger$ Stefano Tomassi, ${ }^{b, \ddagger}$ Ali M. Yousif, ${ }^{a}$ Anna Messere, ${ }^{b}$ Luciana Marinelli, ${ }^{a}$ Paolo Grieco, ${ }^{a}$ Ettore Novellino, ${ }^{a}$ Sandro Cosconati,${ }^{b, *}$ and Salvatore Di Maro ${ }^{b, *}$

${ }^{a}$ Dipartimento di Farmacia, Università degli Studi di Napoli "Federico II”, via D. Montesano 49, 80131 Naples (Italy); ${ }^{b}$ DiSTABiF, Università degli Studi della Campania “Luigi Vanvitelli”, via A. Vivaldi 43, 81100 Caserta (Italy).

These authors contributed equally.

\section{Table of Contents}

1. Experimental Procedures $\quad$ S2

$\begin{array}{ll}\text { 1.1 Materials and general procedures } & \text { S2 }\end{array}$

1.2 Aluminum foil test $\quad$ S3

1.3 Scanning Electron Microscopy (SEM) S4

1.4 US-SPPS: detailed manual steps $\quad$ S5

2. Optimization of Reaction Conditions $\quad$ S7

$\begin{array}{ll}2.1 \text { Fmoc-deprotection } & \text { S7 }\end{array}$

2.2 Aspartimide study $\quad$ S9

2.3 Coupling (Table 1, entries 1-12) S9

2.4 Racemization degree (Table 2, entries 1-8) $\quad$ S12

2.5 Longer sequences (Table 3, entries 1-9) $\quad \mathrm{S} 13$

$\begin{array}{ll}2.6 \text { Difficult sequences } & \text { S14 }\end{array}$

3. HPLC Chromatograms and Mass Spectra $\quad$ S17

4. References $\quad$ S52 


\section{Experimental Procedures}

\subsection{Materials and general procedures}

The ultrasonic bath SONOREX RK $52 \mathrm{H}$ (interior dimensions $150 \times 140 \times 100 \mathrm{~mm}$ and operating volume 1.2 L) by BANDELIN electronic (Germany), equipped with timer control for 1-15 minutes and continuous $(\infty)$ operations and built-in heating control $\left(30-80{ }^{\circ} \mathrm{C}\right.$ thermostatically adjustable), was employed to produce ultrasonic irradiation. Ultrasonic frequency is $35 \mathrm{kHz}$. The ultrasonic nominal output is $60 \mathrm{~W}$. The ultrasonic peak output is $240 \mathrm{~W}$ and corresponds to 4 times ultrasonic nominal output. The heating power is $140 \mathrm{~W}$.

All standard $N^{\alpha}$-Fmoc amino acids used [Fmoc-L-Ala-OH, Fmoc-L-Arg(Pbf)-OH, Fmoc-L-Asn(Trt)-OH, Fmoc-L-Asp(OtBu)-OH, Fmoc-L-Cys(Trt)-OH, Fmoc-L-Gln(Trt)-OH, Fmoc-L-Glu(OtBu)-OH, Fmoc-LGly-OH, Fmoc-L-His(Trt)-OH, Fmoc-L-Ile-OH, Fmoc-L-Leu-OH, Fmoc-L-Lys(Boc)-OH, Fmoc-L-Met$\mathrm{OH}, \quad$ Fmoc-L-Phe-OH, Fmoc-L-Pro-OH, Fmoc-L-Ser( $t \mathrm{Bu})-\mathrm{OH}, \quad$ Fmoc-L-Thr $(t \mathrm{Bu})-\mathrm{OH}, \quad$ Fmoc-L$\operatorname{Trp}(\mathrm{Boc})-\mathrm{OH}$, Fmoc-L-Tyr( $t \mathrm{Bu})-\mathrm{OH}$, Fmoc-L-Val-OH] were from AAPPTec (Louisville, USA). The unconventional amino acid Fmoc-Aib-OH was purchased from Iris-Biotech GmbH (Marktredwitz, Germany). Piperidine (peptide grade, purity 99.9\%), trifluoroacetic acid (TFA) (peptide grade, purity 99.9\%), triisopropylsilane (TIS) (purity >97\%) and $N, N$-diisopropylethylamine (DIPEA) (purity 99.7\%) were purchased from Iris-Biotech $\mathrm{GmbH}$ (Marktredwitz, Germany). Coupling reagents such as $N, N, N^{\prime}, N^{\prime}$-tetramethyl- $O$-(1H-benzotriazol-1-yl)uronium hexafluorophosphate (HBTU) (purity $\geq 98.0 \%$ ), 1-hydroxybenzotriazole (HOBt) (purity $>97 \%$ dry weight, water $\sim 12 \%$ ), $N, N, N^{\prime}, N^{\prime}-$ tetramethyl-O-(6-chloro-1H-benzotriazol-1-yl)uronium hexafluorophosphate (HCTU) (purity $\geq 98 \%$ ), hydroxy-7-azabenzotriazole (HOAt) (purity $\geq 97.0 \%$ ), N,N'-Diisopropylcarbodiimide (DIC) (purity $99 \%)$ 3-hydroxy-3H-1,2,3-triazolo[4,5-b]pyridinato- $O$ )tri-1-pyrrolidinyl-phosphorus hexafluorophosphate (PyAOP), 6-chloro-benzotriazole-1-yloxy-tris-pyrrolidinophosphonium hexafluorophosphate (PyClock), 1-cyano-2-ethoxy-2-oxoethylidenaminooxy)dimethylaminomorpholino-carbenium hexafluorophosphate (COMU) (purity 97\%), ethyl cyano(hydroxyimino)acetate (Oxyma) (purity 97\%), all were purchased by Sigma-Aldrich/Merck (St. Louis, USA). Acetic anhydride $\left(\mathrm{Ac}_{2} \mathrm{O}\right.$, purity $\left.>98 \%\right)$ and hexafluoropropanol (HFIP) were purchased from Sigma-Aldrich (Milano, Italy). The resins Fmoc Rink amide (aminomethyl)polystyrene (AM-PS) (0.74 mmol/g as loading, 100200 mesh as particle size; $0.55 \mathrm{mmol} / \mathrm{g}$ as loading, 100-200 mesh as particle size; $0.40 \mathrm{mmol} / \mathrm{g} \mathrm{mmol} / \mathrm{g}$ as loading, 100-200 mesh as particle size; $0.22 \mathrm{mmol} / \mathrm{g} \mathrm{mmol} / \mathrm{g}$ as loading, 100-200 mesh as particle size), Fmoc-L-Leu-Wang (0.99 mmol/g as loading, 100-200 mesh as particle size), and Fmoc-L-Arg(Pbf)2-chlorotrityl chloride (2-CTC, $0.74 \mathrm{mmol} / \mathrm{g}$ as loading, 100-200 mesh as particle size), all were purchased by Iris-Biotech GmbH (Marktredwitz, Germany), and the manufacturer's reported loading of the resin was used in the calculations. Solvents for peptide synthesis and analysis such as $N, N$ - 
dimethylformamide (DMF), dichloromethane (DCM), water and acetonitrile (MeCN) for HPLC, and diethyl ether $\left(\mathrm{Et}_{2} \mathrm{O}\right)$ were reagent grade and acquired from commercial sources (Sigma-Aldrich/Merck, Milano, Italy). Unless noted, all solvents were not anhydrous and used without further purification. After each coupling and Fmoc-deprotection reactions the resin was washed with DMF $(3 \times 2 \mathrm{~mL})$ and DCM ( 3 $\times 2 \mathrm{~mL}$ ), prior to be monitored by the observation of specific colorimetric tests. In particular, a few dried beads were assayed by the Kaiser/TNBS or $p$-chloranil tests for the presence of primary and secondary amines, respectively. ${ }^{1-3}$

Analytical HPLC analyses were performed by reverse-phase HPLC (Shimadzu Prominance UFLC, SPDM20A/DGU-20A3R/LC-20AD) on a Phenomenex Kinetex column $(\mathrm{C} 18,150 \mathrm{~mm} \times 4.6 \mathrm{~mm}, 5 \mu \mathrm{m}, 100$ $\AA$ A) with a flow rate of $1 \mathrm{~mL} / \mathrm{min}$, with detection at 220 and $254 \mathrm{~nm}$ wavelengths by a diode array UV-Vis detector, and by using different gradient elutions of $\mathrm{MeCN}(0.1 \% \mathrm{TFA})$ in water $(0.1 \% \mathrm{TFA})$ [Gradient 1: isocratic $10 \% \mathrm{MeCN}\left(0.1 \%\right.$ TFA) in $\mathrm{H}_{2} \mathrm{O}\left(0.1 \%\right.$ TFA) over $5 \mathrm{~min}, 10-90 \% \mathrm{MeCN}\left(0.1 \%\right.$ TFA) in $\mathrm{H}_{2} \mathrm{O}$ (0.1\% TFA) over 20 min; Gradient 2: isocratic $100 \% \mathrm{H}_{2} \mathrm{O}(0.1 \%$ TFA) over $5 \mathrm{~min}, 0-40 \% \mathrm{MeCN}(0.1 \%$ TFA) in $\mathrm{H}_{2} \mathrm{O}$ (0.1\% TFA) over 30 min; Gradient 3: isocratic $10 \% \mathrm{MeCN}\left(0.1 \%\right.$ TFA) in $\mathrm{H}_{2} \mathrm{O}(0.1 \%$ TFA) over 5 min, $10-60 \% \mathrm{MeCN}\left(0.1 \%\right.$ TFA) in $\mathrm{H}_{2} \mathrm{O}$ (0.1\% TFA) over $20 \mathrm{~min}$; Gradient 4: isocratic $0 \% \mathrm{MeCN}$ $\left(0.1 \% \mathrm{NH}_{4} \mathrm{OH}\right)$ in $\mathrm{H}_{2} \mathrm{O}\left(0.1 \% \mathrm{NH}_{4} \mathrm{OH}\right)$ over 5 min, $0-60 \% \mathrm{MeCN}\left(0.1 \% \mathrm{NH}_{4} \mathrm{OH}\right)$ in $\mathrm{H}_{2} \mathrm{O}\left(0.1 \% \mathrm{NH}_{4} \mathrm{OH}\right)$ over $20 \mathrm{~min}$ ]. Mass measurements were acquired by LC/MS system (LCMS-2020, Shimadzu) at the flow rate of $0.2 \mathrm{~mL} / \mathrm{min}$, and proton adducts, $[\mathrm{M}+\mathrm{H}]^{+}$, were used for empirical formula confirmation. Temperature control was carried out by the external immersion cooler TC100E by Huber (Germany).

\subsection{Aluminum foil test}

For our studies, an ultrasonic cleaning bath operating at frequency of $35 \mathrm{KHz}$ was employed. As one of the most widely available sources of ultrasound waves for the chemical laboratories, this apparatus generates radiations with a frequency value belonging to the recognized interval of sonochemistry (20$100 \mathrm{KHz}$ ), enabling at the same time the use of common polypropylene vessels for SPPS without any specific adaptation. However, to guarantee both efficiency and reproducibility of the chemical reactions, the low homogeneity of the wave propagation inside the bath must be taken into account. ${ }^{4}$ Ideally, the reaction vessel should be placed at the point where the maximum sonochemical effects are observed. To identify these areas, we applied the "aluminium foil test", which is recommended by the vendors of ultrasonic baths as qualitative procedure to monitor the intensity and distribution of ultrasound waves. An aluminium foil (thickness between 0.01 and $0.025 \mathrm{~mm}$ ) was cut to fit the bath and placed in it. Then, sonication was carried out for $60 \mathrm{sec}$. After this time interval the foil presented perforated areas, which corresponded to the zones with maximum cavitation intensity. To assure reproducible results it is important to provide same testing conditions, such as the liquid level in the bath and the operating 
temperature. Moreover, the aluminum foil test is recommended to be executed at initial runs, and at regular intervals thereafter.
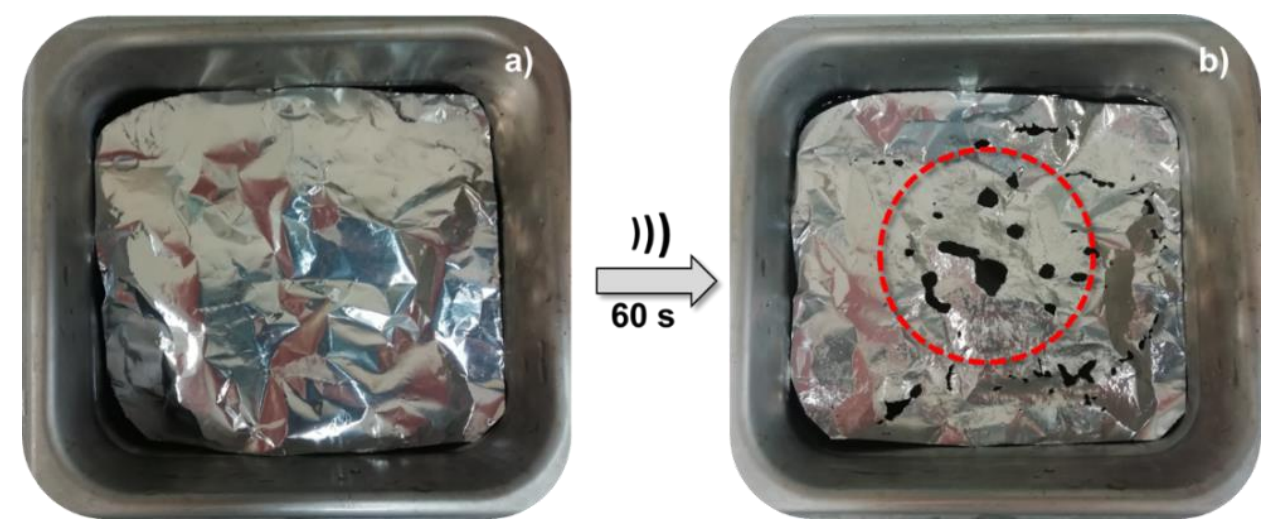

Figure S1. The aluminum foil inside the ultrasonic bath before sonication (a), and after $60 \mathrm{sec}$ of ultrasonic irradiation (b).

\subsection{Scanning Electron Microscopy (SEM)}

Considering the well-known capability of cavitation to erode the solid surface contacting the fluid, ${ }^{4}$ we examined the integrity of the solid support upon ultrasonic irradiation. A suspension of Fmoc Rink amide AM-PS resin (1\% divinylbenzene crosslinking) in $N, N$-dimethylformamide (DMF) was sonicated or mechanically shaken for $60 \mathrm{~min}$, which is the time required to perform a coupling reaction by conventional strategy. Subsequently, the suspension was dried and the beads visualized by Scanning Electron Microscopy (SEM) performed with a bench top Phenom XL by Thermo Fisher Scientific (Alfatest, Milan, Italy) following manufacturer instructions. As displayed in Figure S2, the sonicated beads (Figure S2a) appeared to be intact, and were similar to those treated by mechanical shaking (Figure S2b), indicating that low-frequency ultrasonication does not affect the overall polymeric structure of the resin and can be safely investigated without determining the undesired release of resin fragments from the solid support.
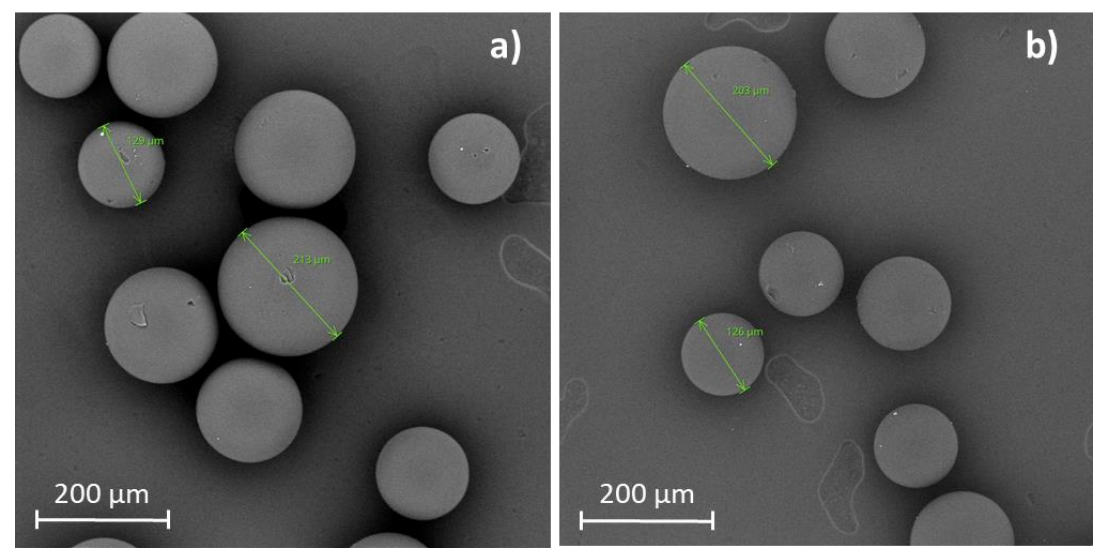

Figure S2. SEM images of dried Rink amide (aminomethyl)polystyrene (AM-PS) resin beads treated by

(a) $60 \mathrm{~min}$ mechanical shaking at $200 \mathrm{rpm}$ or (b) $60 \mathrm{~min}$ ultrasonic (35 KHz) exposure. 


\subsection{US-SPPS: detailed manual steps}

Peptides assembled by US-SPPS were manually synthesized by using the lab amenities depicted in Figure S3. In particular, each peptide sequence was assembled on a Fmoc-Rink-Amide AM resin as solid support that was first placed into a $10 \mathrm{~mL}$ polypropylene tube (Figure S3a) (ISOLUTE ${ }^{\circledR}$ SPE filtration column by Biotage, Uppsala, Sweden) equipped with filter (ISOLUTE ${ }^{\circledR}$ frits, $20 \mu \mathrm{m}$ porosity polyethylene frits by Biotage, Uppsala, Sweden), stopper, and top cap. Prior to use, the appropriate amount of resin was swollen for $30 \mathrm{~min}$ at $\mathrm{rt}$ on a magnetic stirrer plate (Figure S3c) (MR Hei-Standard by Heidolph, Schwabach, Germany). Then, a solid-phase extraction (SPE) vacuum manifold (Figure S3d) (Phenomenex, Torrance, USA) was used for filtering and washing procedures of the resin by means of a PTFE universal stopcock (Figure S3a) that allowed the solvent passing through the resin and collected in a waste container.

The synthetic route consisted of cycles of peptide bond formation (couplings) and $N^{\alpha}$-Fmoc removal (Fmoc-deprotections) reactions, both performed by the ultrasonic bath (Figure S3e) (SONOREX RK 52 $\mathrm{H}$ by BANDELIN electronic, Germany). The ultrasonic irradiation to perform SPPS reactions was performed by placing the polypropylene vessel in the ultrasonic bath with reaction mixture not exceeding the water level. Considering that heating processes originated by the operating system could mislead the outcome evaluation, an external thermometer was employed to assure that the temperature was kept between $25-30{ }^{\circ} \mathrm{C}$, before and after each reaction step. During the experiments where US-SPPS was performed at 5, 10 and $15{ }^{\circ} \mathrm{C}$ (Table 1 , entries 10, 11 and 12, respectively), the temperature of the ultrasonic bath water was chilled by an external immersion cooler equipped with temperature control (Figure S3b). The procedure to remove the excess of reagents (filtering and washing) was carried out by the SPE vacuum manifold.

Upon the construction of the resin-bound peptide sequences, these were thoroughly washed and dried. Hence, the peptides were cleaved by treating with TFA/TIS/ $\mathrm{H}_{2} \mathrm{O}(95: 2.5: 2.5 v / v / v)$ on magnetic stirrer plate $(200 \mathrm{rpm})$, recovered by precipitation with chilled anhydrous $\mathrm{Et}_{2} \mathrm{O}(10 \mathrm{~mL})$, and then centrifuged $(6000 \mathrm{rpm} \times 15 \mathrm{~min})$. The supernatants were carefully removed and the resulting white to pale beigecolored amorphous solids were dried and dissolved in water/acetonitrile (9:1) to be analyzed by reversephase HPLC. 


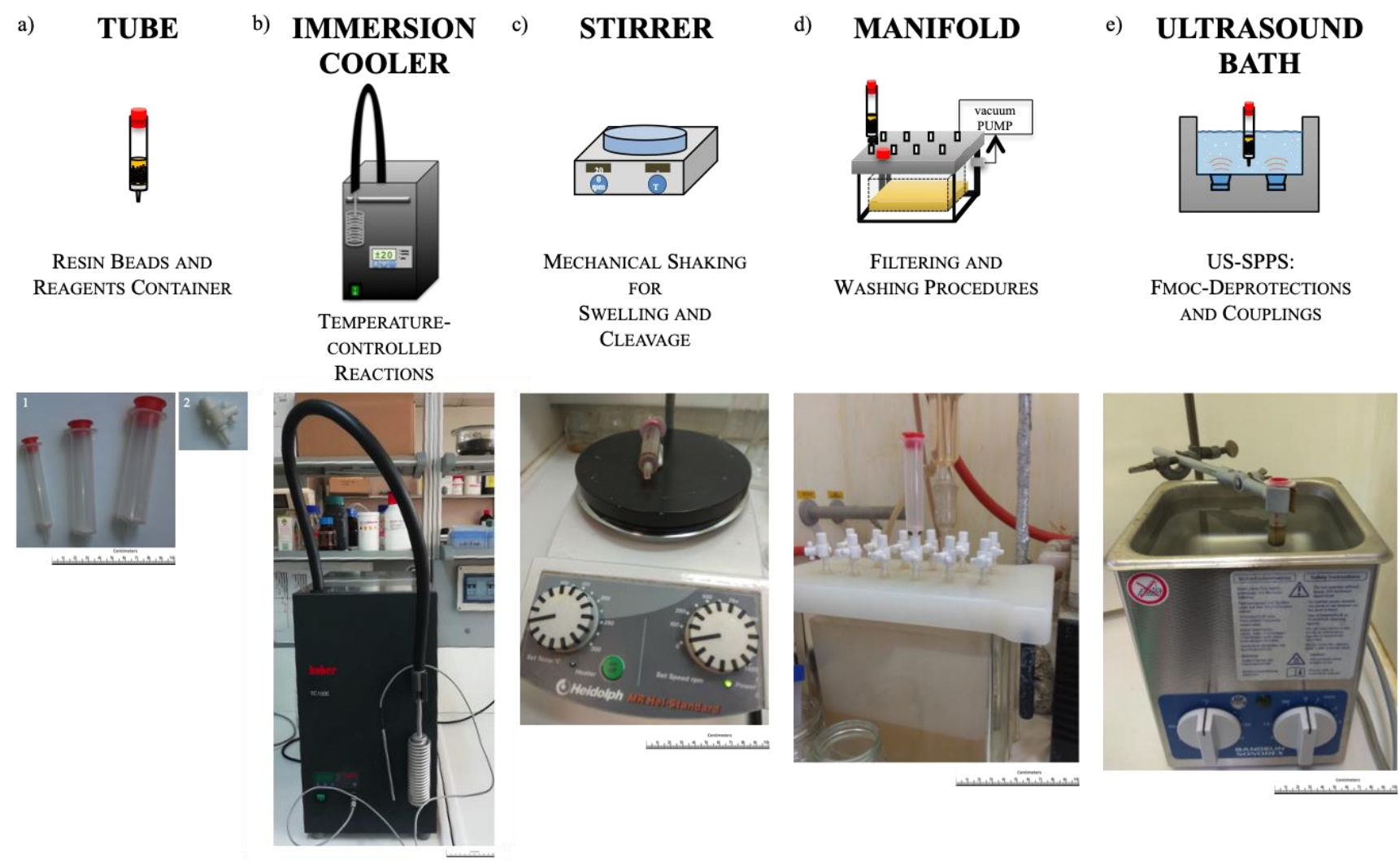

Figure S3. The operating system used for the US-SPPS: a) polypropylene tube equipped with filter, top cap, and stopper (1) or PTFE universal stopcock (2) when needed; b) immersion cooler used for controlling the temperature in specific investigations; c) magnetic stirrer plate; d) vacuum manifold for solid-phase extraction (SPE); e) ultrasonic bath equipped with timer control for 1-15 minutes and continuous $(\infty)$ operations and built-in heating control $\left(30-80{ }^{\circ} \mathrm{C}\right.$ thermostatically adjustable). 


\section{Optimization of Reaction Conditions}

\subsection{Fmoc-deprotection}

$\boldsymbol{U V}$ spectroscopic analysis. For quantification of dibenzofulvene-piperidine adduct, UV absorbance spectra were recorded using an Evolution $300 \mathrm{UV}-\mathrm{Vis}$ spectrophotometer (Thermo Scientific) equipped with a Peltier temperature controller. Prior to the spectroscopic analysis, $68 \mathrm{mg}$ of Fmoc Rink amide AMPS resin $(0.05 \mathrm{mmol})$ was placed in a dried $3 \mathrm{~mL}$ polypropylene vessel, swelled in $3 \mathrm{~mL}$ of dry DMF for $30 \mathrm{~min}$, and drained.

Immediately prior to the Fmoc removal, an aliquot of dried resin bound Fmoc-Asp $(\mathrm{O} t \mathrm{Bu}$ ) (approximately $5 \mu \mathrm{mol}$ with the regard to experimental resin substitution) was placed into a $3 \mathrm{~mL}$ polypropylene tube equipped with filter, stopper, and top cap. Then, the pre-swollen resin was treated with $1 \mathrm{~mL}$ of a piperidine solution (20\% piperidine in DMF, procedures A and B) and exposed to ultrasonic irradiation at different time intervals (1-5). The resulting heterogeneous reaction mixture was filtered in a $10 \mathrm{~mL}$ graduated flask and the filtrate was diluted to $10 \mathrm{~mL}$ with fresh DMF. $1 \mathrm{~mL}$ of $20 \%$ piperidine in DMF solution without the addition of the resin was equally prepared as blank. A volume of $500 \mu \mathrm{L}$ of experimental solution was transferred to an UV quartz cell $(3 \times 10 \mathrm{~mm})$ and placed in an UV-Vis spectrophotometer (Quartz cuvette with optical path length $=1 \mathrm{~cm}$ ), and optical density was recorded at wavelengths ranging from 200 to $400 \mathrm{~nm}$. Fulvene-piperidine adduct was monitored at $301 \mathrm{~nm}$ (extinction coefficient $\varepsilon=7800 \mathrm{~mL} \mathrm{mmol}^{-1} \mathrm{~cm}^{-1}$ ). Finally, the Fmoc-loading quantification was calculated according to the following formula: Fmoc-loading $(\mathrm{mol} / \mathrm{g})=\left[\left(\mathrm{A}_{\text {sample }}-\mathrm{A}_{\text {ref }}\right) / 7800 \times 1 \mathrm{~cm}\right] \times[10 \mathrm{~mL} / \mathrm{mg}$ of resin $]$.

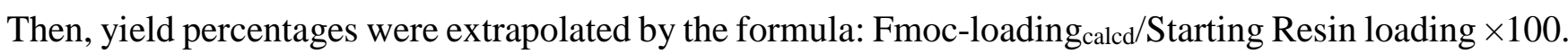
Experiments were performed in triplicate and the mean effective Fmoc removal percentages are expressed as the mean \pm S.E.M. (Table S1).

Table S1. Ultrasound-assisted Fmoc-deprotection: rounds of optimization.

$\begin{array}{ccccc}\text { entry } & t(\mathrm{~min}) & \text { procedure }^{a} & \operatorname{temp~}^{b}\left({ }^{\circ} \mathrm{C}\right) & \text { yield }(\%) \\ \mathbf{1} & 2+10 & \mathrm{~A} & 44.6 \pm 0.8 & >99 \\ \mathbf{2} & 1+5 & \mathrm{~A} & 43.7 \pm 1.5 & >99 \\ \mathbf{3} & 0.5+2 & \mathrm{~A} & 40.0 \pm 1.0 & >99 \\ \mathbf{4} & 0.5+1 & \mathrm{~A} & 35.1 \pm 1.1 & >99 \\ \mathbf{5} & 0.25+1 & \mathrm{~A} & 34.4 \pm 0.8 & 91 \pm 3 \\ \mathbf{6} & 5+25 & \mathrm{~B} & 25.4 & >99\end{array}$

${ }^{a} \mathrm{~A}=20 \%$ piperidine in $\mathrm{DMF}(v / v)$, by ultrasonic irradiation; $\mathrm{B}=20 \%$ piperidine in $\mathrm{DMF}(v / v)$, by mechanical shaking. ${ }^{b}$ Highest temperature observed for each reaction time. 
Synthesis of 10-mer oligo-alanine peptides. The Ala 10 peptide models (7-9) for Fmoc-deprotection assay were synthesized following standard Fmoc chemistry protocol on a Fmoc Rink amide AM-PS solid support $(0.55 \mathrm{mmol} / \mathrm{g}, 91 \mathrm{mg}, 0.05 \mathrm{mmol})$. Coupling reactions were carried out in SPPS reaction vessel adding to the resin a solution of Fmoc-L-Ala-OH (4 equiv), HBTU (4 equiv), HOBT (4 equiv) and DIPEA (8 equiv) in $500 \mu \mathrm{L}$ of DMF, and then stirring the reaction mixture for $1 \mathrm{~h}$ at rt. Upon DMF washing, 10 min capping step was performed after each coupling reaction by using $0.5 \mathrm{M}$ acetic anhydride and 0.125 M DIPEA in DMF. To test the Fmoc-deprotection efficiency, three different Fmoc-removal protocols were used separately: i) 20\% piperidine in DMF ( $3 \mathrm{~mL}$ ) by ultrasonic irradiation $(0.5+1 \mathrm{~min})$; $i$ i $) 20 \%$ piperidine in DMF (3 mL) by mechanical shaking $(0.5+1 \mathrm{~min})$; iii) $20 \%$ piperidine in $\mathrm{DMF}(3 \mathrm{~mL})$ by mechanical shaking $(5+25 \mathrm{~min})$. After filtration, each resulting solution was collected in separate volumetric flask up to $10 \mathrm{~mL}$ and diluted $(\times 100)$ with $20 \%$ piperidine in DMF. The UV-spectroscopic measurements at $301 \mathrm{~nm}$ of released dibenzofulvene-piperidine adduct allowed us to calculate the Fmocdeprotection yield expressed as percentage of maximum functionalization of the support $(\Psi): \Psi=[(\mathrm{Abs}$. $\mathrm{Vol}) / \varepsilon \cdot 1 \cdot \mathrm{m}] /$ loading $\times 100$, where Abs is Absorbance at $301 \mathrm{~nm}$; Vol is total Volume $(\mathrm{mL})$ of $20 \%$ piperidine in DMF solution; $\varepsilon=$ extinction coefficient of dibenzofulvene-piperidine adduct at $301 \mathrm{~nm}$ $\left(7.8 \mathrm{mmol}^{-1} \mathrm{~cm}^{-1}\right) ; 1=$ path length; $\mathrm{m}$ is the support weight $(\mathrm{mg})$. Experiments were performed in triplicate and the mean effective Fmoc removal percentages are expressed as the mean \pm S.E.M. (Table S2).

Table S2. Fmoc-deprotection yields from oligo-alanine peptides 7-9.

\begin{tabular}{|c|c|c|c|c|c|c|c|c|c|c|}
\hline \multirow{2}{*}{ entry } & \multicolumn{10}{|c|}{$\Psi^{a}(\%)$} \\
\hline & $\mathrm{Ala}^{1}$ & $\mathrm{Ala}^{2}$ & $\mathrm{Ala}^{3}$ & $\mathrm{Ala}^{4}$ & $\mathrm{Ala}^{5}$ & $\mathrm{Ala}^{6}$ & $\mathrm{Ala}^{7}$ & $\mathrm{Ala}^{8}$ & $\mathrm{Ala}^{9}$ & $\mathrm{Ala}^{10}$ \\
\hline 7 & $96 \pm 3$ & $95 \pm 2$ & $93 \pm 5$ & $92 \pm 2$ & $88 \pm 3$ & $88 \pm 2$ & $72 \pm 3$ & $60 \pm 2$ & $60 \pm 3$ & $70 \pm 3$ \\
\hline 8 & $95 \pm 3$ & $94 \pm 5$ & $94 \pm 4$ & $92 \pm 2$ & $88 \pm 4$ & $60 \pm 5$ & $38 \pm 4$ & $32 \pm 5$ & $32 \pm 6$ & $40 \pm 4$ \\
\hline 9 & $97 \pm 2$ & $96 \pm 2$ & $93 \pm 4$ & $95 \pm 2$ & $88 \pm 3$ & $90 \pm 3$ & $60 \pm 2$ & $50 \pm 4$ & $50 \pm 4$ & $54 \pm 2$ \\
\hline
\end{tabular}

${ }^{a}$ Values are referred to the percentage of maximum functionalization of the support after each alanine coupling and Fmoc-deprotection.

Methods for Fmoc-deprotection. The resin-bound Fmoc protecting group was removed according to one of the following three procedures: A) $20 \%$ piperidine solution in DMF, ultrasonic irradiation at different time intervals $(2+10,1+5,0.5+2,0.5+1,0.25+1 \mathrm{~min})$; B) $20 \%$ piperidine solution in $\mathrm{DMF}$, mechanical shaking $(0.5+1,5+25 \mathrm{~min})$; C) $20 \%$ piperidine in $\mathrm{DMF}+1 \%$ formic acid, ultrasonic irradiation $(0.5+1 \mathrm{~min})$. 


\subsection{Aspartimide study}

The 1-6 fragment of toxin II by Scorpion Androctonus australis Hector (VKDGYI) was synthesized on a Rink amide resin $(0.74 \mathrm{mmol} / \mathrm{g} ; 68 \mathrm{mg}, 0.05 \mathrm{mmol})$ by applying three deprotection protocols (entries 1-3). Expected product for entries 1-3: $\mathrm{t}_{\mathrm{R}}=11.1 \mathrm{~min}$ [analytical HPLC/Gradient 1]; ESI-MS $\mathrm{m} / \mathrm{z}$ calcd for molecular formula $\mathrm{C}_{32} \mathrm{H}_{53} \mathrm{~N}_{8} \mathrm{O}_{9}{ }^{+}[\mathrm{M}+\mathrm{H}]^{+}$693.39, found 693.35.

Entry 1. VKDGYI. Fmoc-deprotection: procedure B $(5+25 \mathrm{~min})$. Coupling: $N^{\alpha}$-Fmoc amino acid equiv: 4; coupling reagents (equiv): HBTU/HOBt (4 equiv); DIEA (8 equiv); mechanical shaking (60 $\min )$. Purity $93.0 \%$.

Entry 2. VKDGYI. Fmoc-deprotection: procedure A $(0.5+1 \mathrm{~min})$. Coupling: $N^{\alpha}$-Fmoc amino acid equiv: 4; coupling reagents (equiv): HBTU/HOBt (4 equiv); DIEA (8 equiv); mechanical shaking (60 min). Purity $96.0 \%$.

Entry 3. VKDGYI. Fmoc-deprotection: procedure C. Coupling: $N^{\alpha}$-Fmoc amino acid equiv: 4; coupling reagents (equiv): HBTU/HOBt (4 equiv); DIEA (8 equiv); mechanical shaking (60 min). Purity 97.5\%.

\subsection{Coupling (Table 1, entries 1-12)}

Reaction time and reagents equiv. Entries 1-12 (Table 1) were synthesized on a Rink amide resin (0.74 $\mathrm{mmol} / \mathrm{g} ; 68 \mathrm{mg}, 0.05 \mathrm{mmol}$ ) in triplicate and the mean effective purity values are expressed as the mean \pm S.E.M. Expected product for entries 1-12: $\mathrm{t}_{\mathrm{R}}=17.1 \mathrm{~min}$ [analytical HPLC/Gradient 1]; ESI-MS $\mathrm{m} / \mathrm{z}$ calcd for molecular formula $\mathrm{C}_{49} \mathrm{H}_{61} \mathrm{~N}_{10} \mathrm{O}_{9}{ }^{+}[\mathrm{M}+\mathrm{H}]^{+}$933.46, found 933.25.

The amide reaction was accomplished by adding the corresponding $N^{\alpha}$-Fmoc amino acid and appropriate coupling reagents to the reaction mixture suspended in DMF $(0.13 \mathrm{M})$ by using one of the following protocols.

Entry 1. Fmoc-deprotection: procedure B $(5+25 \mathrm{~min})$. Coupling: $N^{\alpha}$-Fmoc amino acid equiv: 4; coupling reagents (equiv): HBTU/HOBt (4 equiv); base (equiv): DIEA (8 equiv); mechanical shaking (60 min). Purity $84 \pm 3 \%$.

Entry 2. Fmoc-deprotection: procedure A $(0.5+1 \mathrm{~min})$. Coupling: $N^{\alpha}$-Fmoc amino acid equiv: 4; coupling reagents (equiv): HBTU/HOBt (4 equiv); base (equiv): DIEA (8 equiv); ultrasonic irradiation (30 min). Purity $93 \pm 4 \%$.

Entry 3. Fmoc-deprotection: procedure A $(0.5+1 \mathrm{~min})$. Coupling: $N^{\alpha}$-Fmoc amino acid equiv: 4; coupling reagents (equiv): HBTU/HOBt (4 equiv); base (equiv): DIEA (8 equiv); ultrasonic irradiation (10 min). Purity $92 \pm 3 \%$.

Entry 4. Fmoc-deprotection: procedure A $(0.5+1 \mathrm{~min})$. Coupling: $N^{\alpha}$-Fmoc amino acid equiv: 4; coupling reagents (equiv): HBTU/HOBt (4 equiv); base (equiv): DIEA (8 equiv); ultrasonic irradiation (5 min). Purity $90 \pm 2 \%$. 
Entry 5. Fmoc-deprotection: procedure A $(0.5+1 \mathrm{~min})$. Coupling: $N^{\alpha}$-Fmoc amino acid equiv: 4; coupling reagents (equiv): HBTU/HOBt (4 equiv); base (equiv): DIEA (8 equiv); ultrasonic irradiation (2 min). Purity $76 \pm 4 \%$.

Entry 6. Fmoc-deprotection: procedure A $(0.5+1 \mathrm{~min})$. Coupling: $N^{\alpha}$-Fmoc amino acid equiv: 3 ; coupling reagents (equiv): HBTU/HOBt (3 equiv); base (equiv): DIEA (6 equiv); ultrasonic irradiation (5 min). Purity $89 \pm 2 \%$.

Entry 7. Fmoc-deprotection: procedure A $(0.5+1 \mathrm{~min})$. Coupling: $N^{\alpha}$-Fmoc amino acid equiv: 2 ; coupling reagents (equiv): HBTU/HOBt (2 equiv); base (equiv): DIEA (4 equiv); ultrasonic irradiation (5 min). Purity $88 \pm 3 \%$.

Entry 8. Fmoc-deprotection: procedure A $(0.5+1 \mathrm{~min})$. Coupling: $N^{\alpha}$-Fmoc amino acid equiv: 1; coupling reagents (equiv): HBTU/HOBt (1 equiv); base (equiv): DIEA (2 equiv); ultrasonic irradiation (5 min). Purity $65 \pm 3 \%$.

Entry 9. Oil bath temperature: $45^{\circ} \mathrm{C}$. Fmoc-deprotection: procedure B $(0.5+1 \mathrm{~min})$. Coupling: $N^{\alpha}{ }_{-}$ Fmoc amino acid equiv: 2; coupling reagents (equiv): HBTU/HOBt (2 equiv); base (equiv): DIEA (4 equiv); mechanical shaking (5 min). Purity $64 \pm 5 \%$.

Entry 10. Ultrasonic bath temperature: $5^{\circ} \mathrm{C}$. Fmoc-deprotection: procedure A $(0.5+1 \mathrm{~min})$. Coupling: $N^{\alpha}$-Fmoc amino acid equiv: 2; coupling reagents (equiv): HBTU/HOBt (2 equiv); base (equiv): DIEA (4 equiv); ultrasonic irradiation ( $5 \mathrm{~min}$ ). Purity $85 \pm 2 \%$.

Entry 11. Ultrasonic bath temperature: $10^{\circ} \mathrm{C}$. Fmoc-deprotection: procedure A ( $\left.0.5+1 \mathrm{~min}\right)$. Coupling: $N^{\alpha}$-Fmoc amino acid equiv: 2; coupling reagents (equiv): HBTU/HOBt (2 equiv); base (equiv): DIEA (4 equiv); ultrasonic irradiation (5 min). Purity $90 \pm 3 \%$.

Entry 12. Ultrasonic bath temperature: $15^{\circ} \mathrm{C}$. Fmoc-deprotection: procedure $\mathrm{A}(0.5+1 \mathrm{~min})$. Coupling: $N^{\alpha}$-Fmoc amino acid equiv: 2; coupling reagents (equiv): HBTU/HOBt (2 equiv); base (equiv): DIEA (4 equiv); ultrasonic irradiation ( $5 \mathrm{~min}$ ). Purity $91 \pm 4 \%$.

Combinations of activating/additive agents. To verify the compatibility and the performance of ultrasonication with the most common activating/additive agents, 2 equiv. reagent excess over 5 min reaction time (entry $\mathbf{7}$, Table 1 ) was applied as the protocol to screen different coupling strategies (Table S3). Six representative activating reagents were employed, including one carbodimmide (DIC), three uronium salts (HBTU, HCTU, COMU) and two phosphonium salts (PyClock and PyAOP) in the presence of three different additive agents, including HOBt, HOAt and Oxyma. 
Table S3. Synthesis of Fmoc-KFRFD by screening common coupling agents for SPPS.

\begin{tabular}{ccc} 
entry & activating/additive agents & $\begin{array}{c}\text { crude } \\
\text { purity }(\%)\end{array}$ \\
\hline $\mathbf{7}$ & HBTU/HOBt & $88 \pm 3$ \\
\hline $\mathbf{7 a}$ & HCTU/HOBt & $86 \pm 2$ \\
$\mathbf{7 b}$ & PyClock/HOBt & $83 \pm 4$ \\
$7 \mathbf{7}$ & COMU/Oxyma & $94 \pm 2$ \\
$7 \mathbf{7 d}$ & PyAOP/HOAt & $88 \pm 4$ \\
$7 \mathbf{7 e}$ & DIC/HOBt & $83 \pm 3$
\end{tabular}

As reported in Table S3, with the exception of the peptides achieved by using PyClock/HOBt (entry 7b) and DIC/HOBt (entry 7e), ultrasonication yielded pentapeptides with a degree of purity comparable or higher than the one achieved in the presence of HBTU/HOBt. Among them, the combination of ultrasonication and COMU/Oxyma allowed for the synthesis of the test peptide with a purity of $94 \pm 2 \%$ (7c), and hence, it was considered as the adopted procedure for all the subsequent experiments. The choice of this coupling strategy was also supported by the recent findings reporting Oxyma as valuable additive agent endowed with a lower risk of explosion and higher capability to suppress racemization compared to HOBt and HOAt. ${ }^{5}$

Entries 7a-e were synthesized on a Rink amide resin $(0.74 \mathrm{mmol} / \mathrm{g}, 68 \mathrm{mg}, 0.05 \mathrm{mmol})$ in triplicate and the mean effective purity values are expressed as the mean \pm S.E.M. Expected product for entries 7a-e: $t_{R}=17.1$ min [analytical HPLC/Gradient 1]; ESI-MS m/z calcd for molecular formula $\mathrm{C}_{49} \mathrm{H}_{61} \mathrm{~N}_{10} \mathrm{O}_{9}{ }^{+}$ $[\mathrm{M}+\mathrm{H}]^{+}$933.46, found 933.25.

The amide reaction was accomplished by adding the corresponding $N^{\alpha}$-Fmoc amino acid and appropriate coupling reagents to the reaction mixture suspended in DMF $(0.13 \mathrm{M})$ by using one of the following protocols

Entry 7a. Fmoc-deprotection: procedure A $(0.5+1 \mathrm{~min})$. Coupling: $N^{\alpha}$-Fmoc amino acid equiv: 2; coupling reagents (equiv): HCTU/HOBt (2 equiv); base (equiv): DIEA (4 equiv); ultrasonic irradiation (5 min). Purity $86 \pm 2 \%$.

Entry 7b. Fmoc-deprotection: procedure A $(0.5+1 \mathrm{~min})$. Coupling: $N^{\alpha}$-Fmoc amino acid equiv: 2; coupling reagents (equiv): PyClock/HOBt (2 equiv); base (equiv): DIEA (4 equiv); ultrasonic irradiation (5 min). Purity $83 \pm 4 \%$. 
Entry 7c. Fmoc-deprotection: procedure A $(0.5+1 \mathrm{~min})$. Coupling: $N^{\alpha}$-Fmoc amino acid equiv: 2 ; coupling reagents (equiv): COMU/Oxyma (2 equiv); base (equiv): DIEA (4 equiv); ultrasonic irradiation (5 min). Purity $94 \pm 2 \%$.

Entry 7d. Fmoc-deprotection: procedure A $(0.5+1 \mathrm{~min})$. Coupling: $N^{\alpha}$-Fmoc amino acid equiv: 2 ; coupling reagents (equiv): PyAOP/HOAt (2 equiv); base (equiv): DIEA (4 equiv); ultrasonic irradiation (5 min). Purity $88 \pm 4 \%$.

Entry 7e. Fmoc-deprotection: procedure A $(0.5+1 \mathrm{~min})$. Coupling: $N^{\alpha}$-Fmoc amino acid equiv: 2 ; coupling reagents (equiv): DIC/HOBt (2 equiv); ultrasonic irradiation (5 min). Purity $83 \pm 3 \%$.

\subsection{Racemization degree (Table 2, entries 1-8)}

Racemization has been long documented as an event occurring during the coupling step and could be a potential concern in the ultrasound-assisted SPPS. To address this issue, eight tripeptides (entries 1-8, Table 2) were synthesized by ultrasound-assisted or conventional strategies using a Rink amide resin $(0.74 \mathrm{mmol} / \mathrm{g} ; 68 \mathrm{mg}, 0.05 \mathrm{mmol})$ and were analyzed by HPLC [Gradient 2].

Synthesis of entries 1, 3, 5 and 7. Fmoc-deprotection: procedure B $(5+25 \mathrm{~min})$; Coupling: $N^{\alpha}$-Fmoc amino acid equiv: 4; coupling reagents (equiv): COMU/Oxyma (4 equiv); base (equiv): DIEA (8 equiv); mechanical shaking (60 $\mathrm{min})$.

Synthesis of 2, 4, 6 and 8. Fmoc-deprotection: procedure A (0.5 $+1 \mathrm{~min})$; Coupling: $N^{\alpha}$-Fmoc amino acid equiv: 2; coupling reagents (equiv): COMU/Oxyma (2 equiv); base (equiv): DIEA (4 equiv); ultrasonic irradiation (5 $\mathrm{min})$.

Entry 1. Purity $96.5 \% ; \mathrm{t}_{\mathrm{R}}=14.9 \mathrm{~min}$ [analytical HPLC/Gradient 2]; ESI-MS $\mathrm{m} / \mathrm{z}$ calcd for molecular formula, $\mathrm{C}_{14} \mathrm{H}_{21} \mathrm{~N}_{4} \mathrm{O}_{3} \mathrm{~S}^{+}[\mathrm{M}+\mathrm{H}]^{+}$325.13, found 325.15.

Entry 2. Purity $94.5 \% ; \mathrm{t}_{\mathrm{R}}=14.9 \mathrm{~min}$ [analytical HPLC/Gradient 2]; ESI-MS $\mathrm{m} / \mathrm{z}$ calcd for molecular formula, $\mathrm{C}_{14} \mathrm{H}_{21} \mathrm{~N}_{4} \mathrm{O}_{3} \mathrm{~S}^{+}[\mathrm{M}+\mathrm{H}]^{+}$325.13, found 325.15.

Entry 3. Purity $97.7 \% ; \mathrm{t}_{\mathrm{R}}=12.4 \mathrm{~min}$ [analytical HPLC/Gradient 2]; ESI-MS $\mathrm{m} / \mathrm{z}$ calcd for molecular formula, $\mathrm{C}_{17} \mathrm{H}_{23} \mathrm{~N}_{6} \mathrm{O}_{3}{ }^{+}[\mathrm{M}+\mathrm{H}]^{+} 359.18$, found 359.15 .

Entry 4. Purity $98.5 \% ; t_{R}=12.4$ min [analytical HPLC/Gradient 2]; ESI-MS $m / z$ calcd for molecular formula, $\mathrm{C}_{17} \mathrm{H}_{23} \mathrm{~N}_{6} \mathrm{O}_{3}{ }^{+}[\mathrm{M}+\mathrm{H}]^{+}$359.18, found 359.15.

Entry 5. Purity $97.3 \% ; t_{R}=17.1 \mathrm{~min}$ [analytical HPLC/Gradient 2]; ESI-MS $\mathrm{m} / \mathrm{z}$ calcd for molecular formula, $\mathrm{C}_{14} \mathrm{H}_{21} \mathrm{~N}_{4} \mathrm{O}_{3} \mathrm{~S}^{+}[\mathrm{M}+\mathrm{H}]^{+}$325.13, found 325.15.

Entry 6. Purity $97.5 \% ; t_{R}=17.1 \mathrm{~min}$ [analytical HPLC/Gradient 2]; ESI-MS $\mathrm{m} / \mathrm{z}$ calcd for molecular formula, $\mathrm{C}_{14} \mathrm{H}_{21} \mathrm{~N}_{4} \mathrm{O}_{3} \mathrm{~S}^{+}[\mathrm{M}+\mathrm{H}]^{+}$325.13, found 325.15.

Entry 7. Purity $>99.0 \% ; \mathrm{t}_{\mathrm{R}}=12.8 \mathrm{~min}$ [analytical HPLC/Gradient 2]; ESI-MS $\mathrm{m} / \mathrm{z}$ calcd for molecular formula, $\mathrm{C}_{17} \mathrm{H}_{23} \mathrm{~N}_{6} \mathrm{O}_{3}{ }^{+}[\mathrm{M}+\mathrm{H}]^{+} 359.18$, found 359.15 . 
Entry 8. Purity >99.0\%; $\mathrm{t}_{\mathrm{R}}=12.8 \mathrm{~min}$ [analytical HPLC/Gradient 2]; ESI-MS $\mathrm{m} / \mathrm{z}$ calcd for molecular formula, $\mathrm{C}_{17} \mathrm{H}_{23} \mathrm{~N}_{6} \mathrm{O}_{3}{ }^{+}[\mathrm{M}+\mathrm{H}]^{+} 359.18$, found 359.15 .

\subsection{Longer sequences (Table 3, entries 1-9)}

Peptides 1, 3, 5, 7-9 (on a Rink amide resin $0.55 \mathrm{mmol} / \mathrm{g}$; $91 \mathrm{mg}, 0.05 \mathrm{mmol}$ ), 2 and $\mathbf{6}$ (on a Wang resin $0.99 \mathrm{mmol} / \mathrm{g}$ pre-loaded with the first amino acid; $50 \mathrm{mg}, 0.05 \mathrm{mmol}$ ), and 4 (on a 2-CTC resin 1.60 $\mathrm{mmol} / \mathrm{g}$ pre-loaded with the first amino acid; $68 \mathrm{mg}, 0.05 \mathrm{mmol}$ ), all were synthesized by adopting reaction conditions employed for 7c [Fmoc-deprotection: procedure A $(0.5+1 \mathrm{~min})$; Coupling: $N^{\alpha}$-Fmoc amino acid equiv: 2; coupling reagents (equiv): COMU/Oxyma (2 equiv); base (equiv): DIEA (4 equiv); ultrasonic irradiation (5 $\mathrm{min})]$.

Entry 1. YNWNSFGLRF. Purity $85 \% ; t_{R}=12.5 \mathrm{~min}$ [analytical HPLC/Gradient 1]; ESI-MS $\mathrm{m} / z$ calcd for molecular formula, $\mathrm{C}_{63} \mathrm{H}_{84} \mathrm{~N}_{17} \mathrm{O}_{14}{ }^{+}[\mathrm{M}+\mathrm{H}]^{+} 1303.47$, found 1303.85 .

Entry 2. DRVYIHPFHL-CO ${ }_{2} \mathrm{H}$. Purity $92 \% ; \mathrm{t}_{\mathrm{R}}=14.9 \mathrm{~min}$ [analytical HPLC/Gradient 1]; ESI-MS $\mathrm{m} / z$ calcd for molecular formula, $\mathrm{C}_{62} \mathrm{H}_{90} \mathrm{~N}_{17} \mathrm{O}_{14}{ }^{+}[\mathrm{M}+\mathrm{H}]^{+} 1296.69$, found 1296.80 and $649.10[(\mathrm{M}+2 \mathrm{H}) / 2]^{+}$.

Entry 3. Ac-SYSMEHFRWGKPV. Acetylation step: $\mathrm{Ac}_{2} \mathrm{O}$ equiv: 4; base (equiv): DIEA (8 equiv); ultrasonic irradiation (5 min). Purity $80 \% ; \mathrm{t}_{\mathrm{R}}=11.0 \mathrm{~min}$ [analytical HPLC/Gradient 1 ; ESI-MS $\mathrm{m} / z$ calcd for molecular formula, $\mathrm{C}_{88} \mathrm{H}_{120} \mathrm{~N}_{23} \mathrm{O}_{20} \mathrm{~S}^{+}[\mathrm{M}+\mathrm{H}]^{+}$1665.92, found $833.40[(\mathrm{M}+2 \mathrm{H}) / 2]^{+}$and 555.85 $[\mathrm{M}+3 \mathrm{H} / 3]^{+}$.

Entry 4. SVTEQGAELSNEER-CO ${ }_{2} \mathrm{H}$. Purity 83\%; $\mathrm{t}_{\mathrm{R}}=11.2 \mathrm{~min}$ [analytical HPLC/Gradient 1]; ESIMS $m / z$ calcd for molecular formula, $\mathrm{C}_{61} \mathrm{H}_{102} \mathrm{~N}_{19} \mathrm{O}_{28}{ }^{+}[\mathrm{M}+\mathrm{H}]^{+} 1548.71$, found $775.00[(\mathrm{M}+2 \mathrm{H}) / 2]^{+}$.

Entry 5. SQETFSDLWKLLPEN. Purity $82 \% ; \mathrm{t}_{\mathrm{R}}=13.1 \mathrm{~min}$ [analytical HPLC/Gradient 1]; ESI-MS $m / z$ calcd for molecular formula $\mathrm{C}_{82} \mathrm{H}_{125} \mathrm{~N}_{20} \mathrm{O}_{26}{ }^{+}[\mathrm{M}+\mathrm{H}]^{+} 1807.02$, found $1807.35[\mathrm{M}+\mathrm{H}]^{+}, 904.00$ $[(\mathrm{M}+2 \mathrm{H}) / 2]^{+}$.

Entry 6. YGGFMTSEKSQTPLVTL-CO ${ }_{2} \mathrm{H}$. Purity $60 \% ; \mathrm{t}_{\mathrm{R}}=16.7 \mathrm{~min}$ [analytical HPLC/Gradient 1]; ESI-MS $m / z$ calcd for molecular formula, $\mathrm{C}_{83} \mathrm{H}_{132} \mathrm{~N}_{19} \mathrm{O}_{27} \mathrm{~S}^{+}[\mathrm{M}+\mathrm{H}]^{+} 1858.93$, found $930.30[(\mathrm{M}+2 \mathrm{H}) / 2]^{+}$.

Entry 7. ARLDVASEFRKKWNKWALSR. Purity 80\%; $\mathrm{t}_{\mathrm{R}}=11.1 \mathrm{~min}$ [analytical HPLC/Gradient 1]; ESI-MS $m / z$ calcd for molecular formula $\mathrm{C}_{112} \mathrm{H}_{179} \mathrm{~N}_{36} \mathrm{O}_{27}{ }^{+}[\mathrm{M}+\mathrm{H}]^{+} 2461.89$, found $1231.35[(\mathrm{M}+2 \mathrm{H}) / 2]^{+}$, $821.20[(\mathrm{M}+3 \mathrm{H}) / 3]^{+}$and $616.15[(\mathrm{M}+4 \mathrm{H}) / 4]^{+}$.

Entry 8. HSDAVFTDNYTRLRKQMAVKKYLNSILN. Purity 63\%; $t_{R}=11.5$ min [analytical HPLC/Gradient 1]; ESI-MS $m / z$ calcd for molecular formula $\mathrm{C}_{147} \mathrm{H}_{239} \mathrm{~N}_{44} \mathrm{O}_{42} \mathrm{~S}^{+}[\mathrm{M}+\mathrm{H}]^{+} 3326.86$, found $1664.30[(\mathrm{M}+2 \mathrm{H}) / 2]^{+}, 1109.75[(\mathrm{M}+3 \mathrm{H}) / 3]^{+}$and $832.60[(\mathrm{M}+4 \mathrm{H}) / 4]^{+}$.

Entry 9. YADAIFTNSYRKVLGQLSARKLLQDIMSRQQGESNQERGARARL. Purity $51 \% ; t_{R}=$ $16.5 \mathrm{~min}$ [analytical HPLC/Gradient 3]; ESI-MS m/z calcd for molecular formula $\mathrm{C}_{215} \mathrm{H}_{359} \mathrm{~N}_{72} \mathrm{O}_{66} \mathrm{~S}^{+}$ 
$[\mathrm{M}+\mathrm{H}]^{+}$5040.74, found $1680.85[(\mathrm{M}+3 \mathrm{H}) / 3]^{+}, 1260.90[(\mathrm{M}+4 \mathrm{H}) / 4]^{+}, 1008.80[(\mathrm{M}+5 \mathrm{H}) / 5]^{+}$and 840.90 $[(\mathrm{M}+6 \mathrm{H}) / 6]^{+}$.

\subsection{Difficult sequences}

The syntheses of the peptides endowed with "difficult sequence": Y-Aib-Aib-FL (Aib-Enkephaline), 1ac; VQAAIDYING (ACP $65-74), \quad \mathbf{2 a - c} \quad$ WFTTLISTIM $\quad(J R \quad 10-m e r), \quad 3 a-c ;$ DAEFRHDSGYEVHHQKLVFFAEDVGSNKGAIIGLMVGGVVIA (A $\beta$ 1-42), 4a-c were performed by modifying the reagent equiv and the reaction temperature (Table S4). All peptides were synthesized on a Rink amide resin $(0.55 \mathrm{mmol} / \mathrm{g}, 91 \mathrm{mg}, 0.05 \mathrm{mmol}$ for $1 \mathbf{a}-\mathbf{c}$ and $\mathbf{2 a - c} ; 0.40 \mathrm{mmol} / \mathrm{g}, 125 \mathrm{mg}, 0.05$ $\mathrm{mmol}$ for 3a-c; $0.22 \mathrm{mmol} / \mathrm{g}, 227 \mathrm{mg}, 0.05 \mathrm{mmol}$ for $\mathbf{4 a - c}$ ) in triplicate and the mean effective purity values are expressed as the mean \pm S.E.M.

Table S4. Optimization study for the synthesis of "difficult sequences": Aib-Enk (entries 1a-c), ACP 65 74 (entries 2a-c), JR 10-mer (entries 3a-c), and A $\beta$ 1-42 (entries 4a-c).

\begin{tabular}{|c|c|c|c|c|c|c|}
\hline ID & entry & procedure $^{a}$ & equiv $^{b}$ & $\begin{array}{c}\text { temp }^{c} \\
\left({ }^{\circ} \mathrm{C}\right)\end{array}$ & purity (\%) & $t(\min )^{d}$ \\
\hline \multirow{3}{*}{ Aib-Enk } & $1 \mathbf{a}$ & A & 4 & $-e$ & $94 \pm 2$ & 59 \\
\hline & $1 b$ & A & 8 & $-e$ & $98 \pm 1$ & 59 \\
\hline & 1c & B & 8 & 45 & $78 \pm 3$ & 59 \\
\hline \multirow{3}{*}{$\mathrm{ACP}_{65-74}$} & $2 \mathbf{a}$ & A & 4 & $-e$ & $78 \pm 2$ & 116.5 \\
\hline & $2 b$ & A & 8 & $-e$ & $91 \pm 2$ & 116.5 \\
\hline & $2 c$ & B & 8 & 45 & $60 \pm 3$ & 116.5 \\
\hline \multirow{3}{*}{ JR10-mer } & $\mathbf{3 a}$ & A & 4 & $-e$ & $66 \pm 2$ & 116.5 \\
\hline & $3 b$ & A & 8 & $-e$ & $75 \pm 1$ & 116.5 \\
\hline & $3 c$ & B & 8 & 45 & $27 \pm 3$ & 116.5 \\
\hline \multirow{3}{*}{$\mathrm{A} \beta 1-42$} & $4 \mathbf{a}$ & A & 4 & $-e$ & $58 \pm 2$ & 484.5 \\
\hline & $4 b$ & A & 8 & $-e$ & $70 \pm 3$ & 484.5 \\
\hline & $4 c$ & $\mathrm{~B}$ & 8 & 45 & $35 \pm 5$ & 484.5 \\
\hline
\end{tabular}

For all peptides the coupling reaction time was $10 \mathrm{~min} .{ }^{a} \mathrm{Fmoc}$-deprotection procedure $\mathrm{A}(0.5+1 \mathrm{~min})$ or B $(0.5+1 \mathrm{~min})$ were employed. ${ }^{b}$ Coupling reagent excess. ${ }^{c}$ Set temperature of the oil bath for the synthesis by mechanical shaking of $\mathbf{1 c}, \mathbf{2 c}, \mathbf{3 c}$ and $\mathbf{4 c} .{ }^{d}$ Overall reactions time needed to accomplish the 
final product. ${ }^{e}$ Highest temperature of the reaction mixture estimated after $10 \mathrm{~min}$ couplings by ultrasonic irradiation was $41.8 \pm 0.7^{\circ} \mathrm{C}$ (see Table 1 , entry 3).

Expected product for entries 1a-c: $t_{\mathrm{R}}=15.5 \mathrm{~min}$ [analytical HPLC/Gradient 1]; ESI-MS $\mathrm{m} / \mathrm{z}$ calcd for molecular formula, $\mathrm{C}_{32} \mathrm{H}_{47} \mathrm{~N}_{6} \mathrm{O}_{6}{ }^{+}[\mathrm{M}+\mathrm{H}]^{+}$611.36, found 611.40.

Entry 1a. Fmoc-deprotection method: procedure A $(0.5+1 \mathrm{~min})$. Coupling: $N^{\alpha}$-Fmoc amino acid equiv: 4; coupling reagents (equiv): COMU/Oxyma (4 equiv); base (equiv): DIEA (8 equiv); ultrasonic irradiation (10 min). Purity $94 \pm 2 \%$.

Entry 1b. Fmoc-deprotection: procedure A $(0.5+1 \mathrm{~min})$. Coupling: $N^{\alpha}$-Fmoc amino acid equiv: 8 ; coupling reagents (equiv): COMU/Oxyma (8 equiv); base (equiv): DIEA (16 equiv); ultrasonic irradiation (10 min). Purity $98 \pm 1 \%$.

Entry 1c. Oil bath temperature: $45^{\circ} \mathrm{C}$. Fmoc-deprotection: procedure B $(0.5+1 \mathrm{~min})$. Coupling: $N^{\alpha}{ }_{-}$ Fmoc amino acid equiv: 8; coupling reagents (equiv): COMU/Oxyma (8 equiv); DIEA (16 equiv); mechanical shaking (10 min). Purity $78 \pm 3 \%$.

Expected product for entries 2a-c: $t_{R}=12.9$ min [analytical HPLC/Gradient 1]; ESI-MS $\mathrm{m} / \mathrm{z}$ calcd for molecular formula, $\mathrm{C}_{47} \mathrm{H}_{76} \mathrm{~N}_{13} \mathrm{O}_{15}{ }^{+}[\mathrm{M}+\mathrm{H}]^{+}$1062.56, found 1062.45.

Entry 2a. Fmoc-deprotection: procedure A $(0.5+1 \mathrm{~min})$. Coupling: $N^{\alpha}$-Fmoc amino acid equiv: 4; coupling reagents (equiv): COMU/Oxyma (4 equiv); base (equiv): DIEA (8 equiv); ultrasonic irradiation (10 min). Purity $78 \pm 2$.

Entry 2b. Fmoc-deprotection: procedure A $(0.5+1 \mathrm{~min})$. Coupling: $N^{\alpha}$-Fmoc amino acid equiv: 8 ; coupling reagents (equiv): COMU/Oxyma (8 equiv); base (equiv): DIEA (16 equiv); ultrasonic irradiation (10 min). Purity $91 \pm 2 \%$.

Entry 2c. Oil bath temperature: $45^{\circ} \mathrm{C}$. Fmoc-deprotection: procedure B $(0.5+1 \mathrm{~min})$. Coupling: $N^{\alpha}{ }_{-}$ Fmoc amino acid equiv: 8; coupling reagents (equiv): COMU/Oxyma (8 equiv); base (equiv): DIEA (16 equiv); mechanical shaking (10 min). Purity $60 \pm 3 \%$.

Expected product for entries 3a-c: $t_{R}=18.1 \mathrm{~min}$ [analytical HPLC/Gradient 1]; ESI-MS $\mathrm{m} / z$ calcd for molecular formula, $\mathrm{C}_{47} \mathrm{H}_{76} \mathrm{~N}_{13} \mathrm{O}_{15}{ }^{+}[\mathrm{M}+\mathrm{H}]^{+}$1211.65, found 1211.60 .

Entry 3a. Fmoc-deprotection: procedure A $(0.5+1 \mathrm{~min})$. Coupling: $N^{\alpha}$-Fmoc amino acid equiv: 4 ; coupling reagents (equiv): COMU/Oxyma (4 equiv); base (equiv): DIEA (8 equiv); ultrasonic irradiation (10 min). Purity $66 \pm 2 \%$.

Entry 3b. Fmoc-deprotection: procedure A $(0.5+1 \mathrm{~min})$. Coupling: $N^{\alpha}$-Fmoc amino acid equiv: 8 ; coupling reagents (equiv): COMU/Oxyma (8 equiv); base (equiv): DIEA (16 equiv); ultrasonic irradiation (10 min). Purity $75 \pm 1 \%$. 
Entry 3c. Oil bath temperature: $45^{\circ} \mathrm{C}$. Fmoc-deprotection: procedure B $(0.5+1 \mathrm{~min})$. Coupling: $N^{\alpha}-$ Fmoc amino acid equiv: 8; coupling reagents (equiv): COMU/Oxyma (8 equiv); base (equiv): DIEA (16 equiv); mechanical shaking (10 min). Purity $27 \pm 3 \%$.

Expected product for entries 4a-c: $\mathrm{t}_{\mathrm{R}}=17.3 \mathrm{~min}$ [analytical HPLC/Gradient 4; sample dissolution in HFIP]; ESI-MS $m / z$ calcd for molecular formula, $\mathrm{C}_{203} \mathrm{H}_{313} \mathrm{~N}_{56} \mathrm{O}_{59} \mathrm{~S}^{+}[\mathrm{M}+\mathrm{H}]^{+} 4511.29$, found 1505.15 $[(\mathrm{M}+3 \mathrm{H}) / 3]^{+}, 1129.10[(\mathrm{M}+4 \mathrm{H}) / 4]^{+}$and $903.25[(\mathrm{M}+5 \mathrm{H}) / 5]^{+}$.

Entry 4a. Fmoc-deprotection: procedure A $(0.5+1 \mathrm{~min})$. Coupling: $N^{\alpha}$-Fmoc amino acid equiv: 4; coupling reagents (equiv): COMU/Oxyma (4 equiv); base (equiv): DIEA (8 equiv); ultrasonic irradiation (10 min). Purity $58 \pm 2 \%$.

Entry 4b. Fmoc-deprotection: procedure A $(0.5+1 \mathrm{~min})$. Coupling: $N^{\alpha}$-Fmoc amino acid equiv: 8 ; coupling reagents (equiv): COMU/Oxyma (8 equiv); base (equiv): DIEA (16 equiv); ultrasonic irradiation (10 min). Purity $70 \pm 3 \%$.

Entry 4c. Oil bath temperature: $45^{\circ} \mathrm{C}$. Fmoc-deprotection: procedure B $(0.5+1 \mathrm{~min})$. Coupling: $N^{\alpha}-$ Fmoc amino acid equiv: 8; coupling reagents (equiv): COMU/Oxyma (8 equiv); base (equiv): DIEA (16 equiv); mechanical shaking (10 min). Purity $35 \pm 5 \%$. 


\section{HPLC Chromatograms and Mass Spectra}

Aspartimide study (Figure 2, entries 1-3)

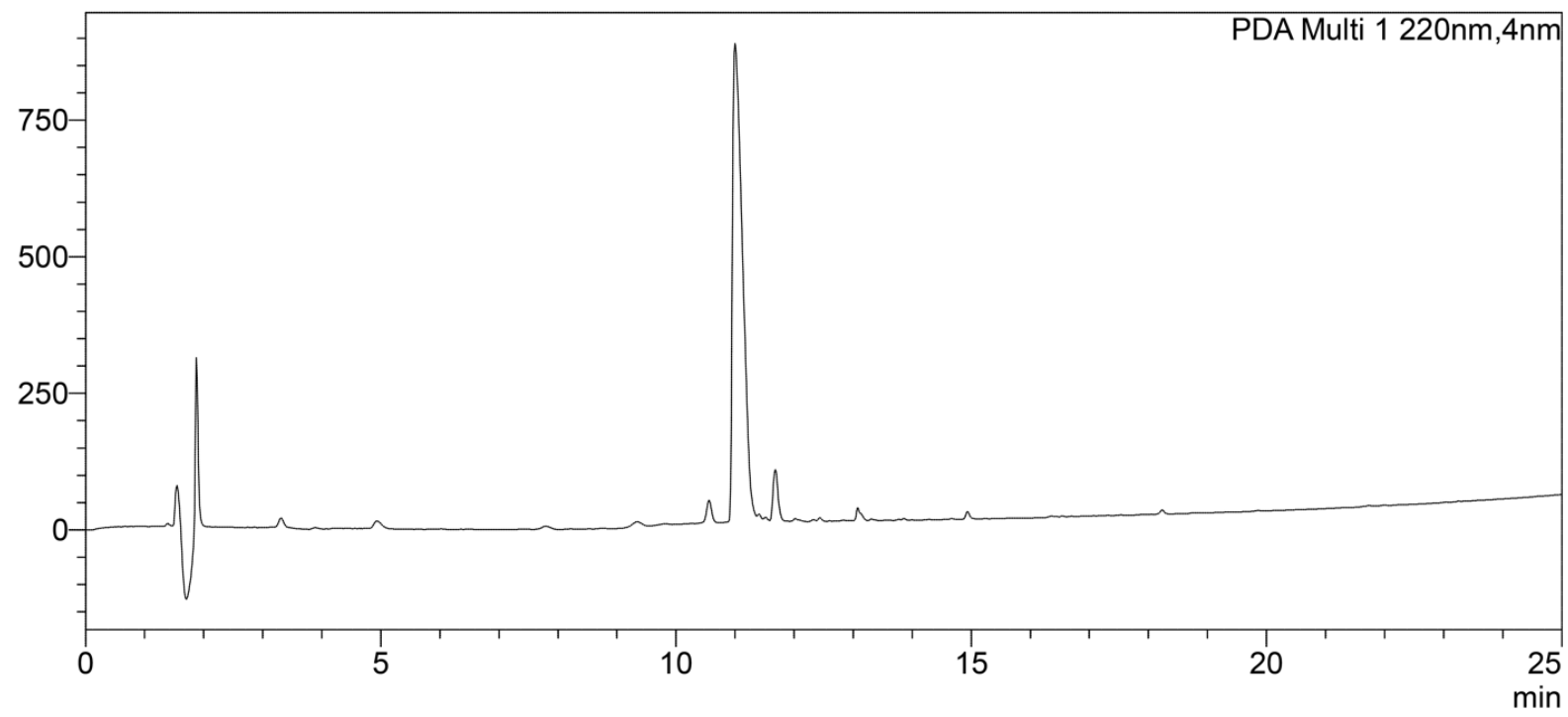

Figure S4. Chromatogram of 1 obtained by RP-HPLC (Shimadzu Prominance UFLC, SPD-M20A/DGU20A3R/LC-20AD) on a Phenomenex Kinetex column $(\mathrm{C} 18,150 \mathrm{~mm} \times 4.6 \mathrm{~mm}, 5 \mu \mathrm{m}, 100 \AA)$. Purity check/Gradient $1-t_{\mathrm{R}}=11.1 \mathrm{~min}$.

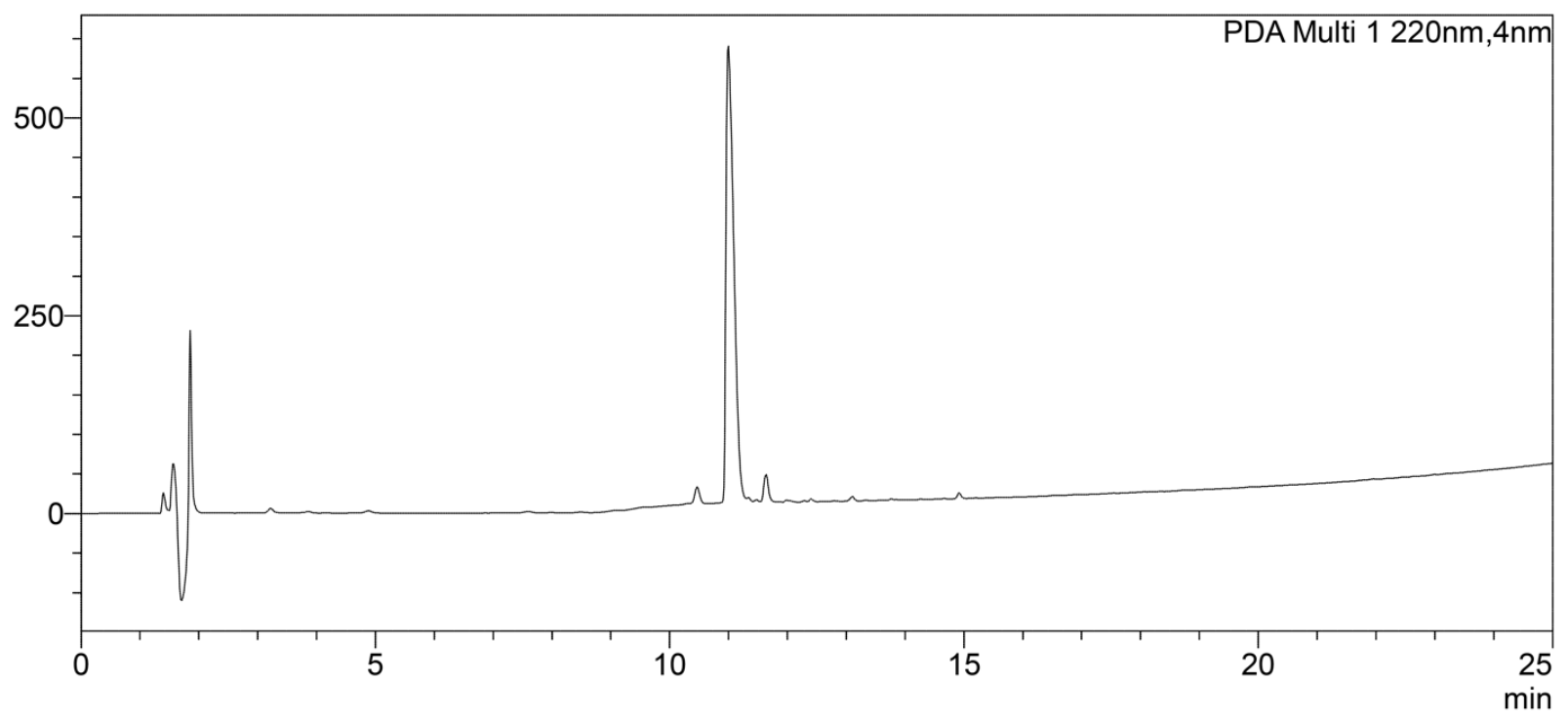

Figure S5. Chromatogram of 2 obtained by RP-HPLC (Shimadzu Prominance UFLC, SPD-M20A/DGU20A3R/LC-20AD) on a Phenomenex Kinetex column $(\mathrm{C} 18,150 \mathrm{~mm} \times 4.6 \mathrm{~mm}, 5 \mu \mathrm{m}, 100 \AA)$. Purity check/Gradient $1-t_{R}=11.1 \mathrm{~min}$. 


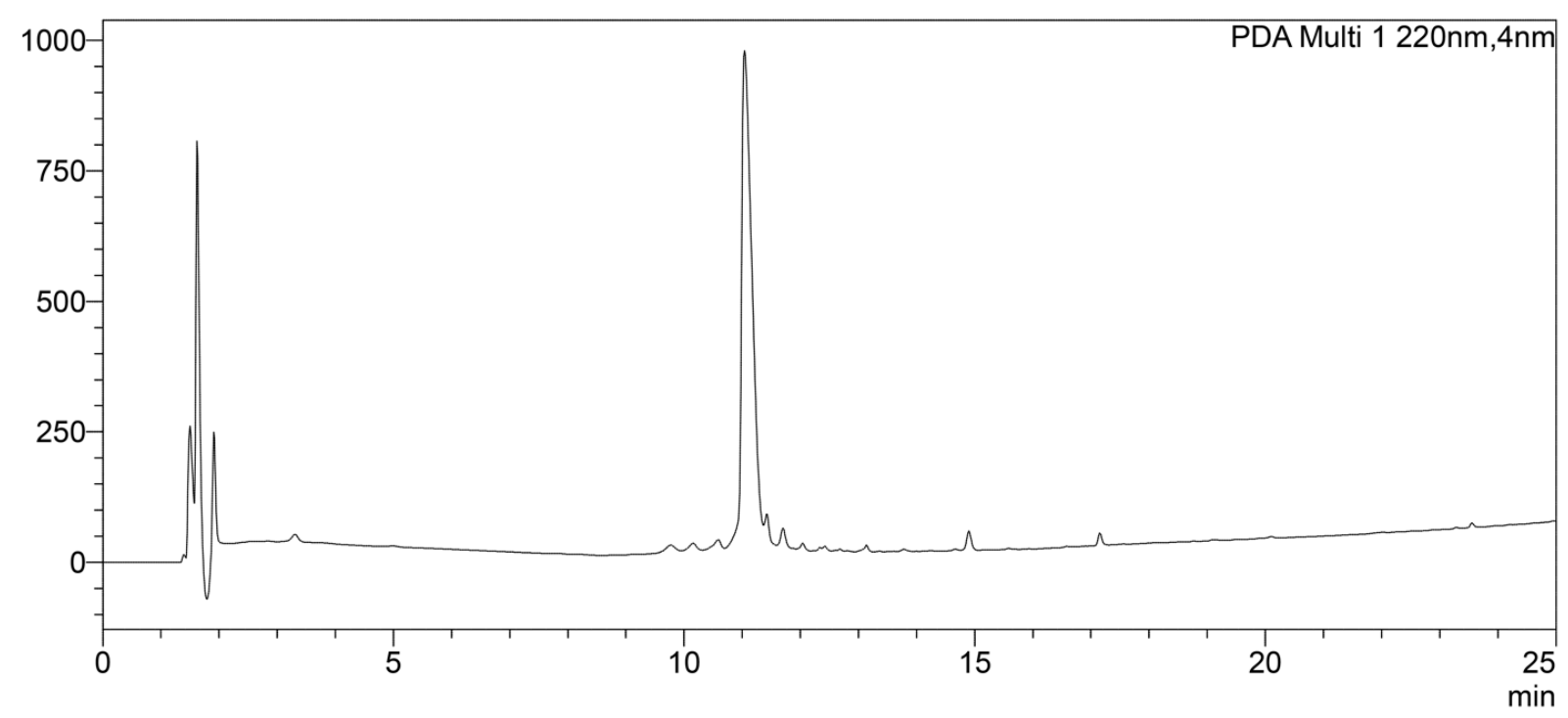

Figure S6. Chromatogram of 3 obtained by RP-HPLC (Shimadzu Prominance UFLC, SPD-M20A/DGU20A3R/LC-20AD) on a Phenomenex Kinetex column $(\mathrm{C} 18,150 \mathrm{~mm} \times 4.6 \mathrm{~mm}, 5 \mu \mathrm{m}, 100 \AA$ ). Purity check/Gradient $1-t_{R}=11.1 \mathrm{~min}$.

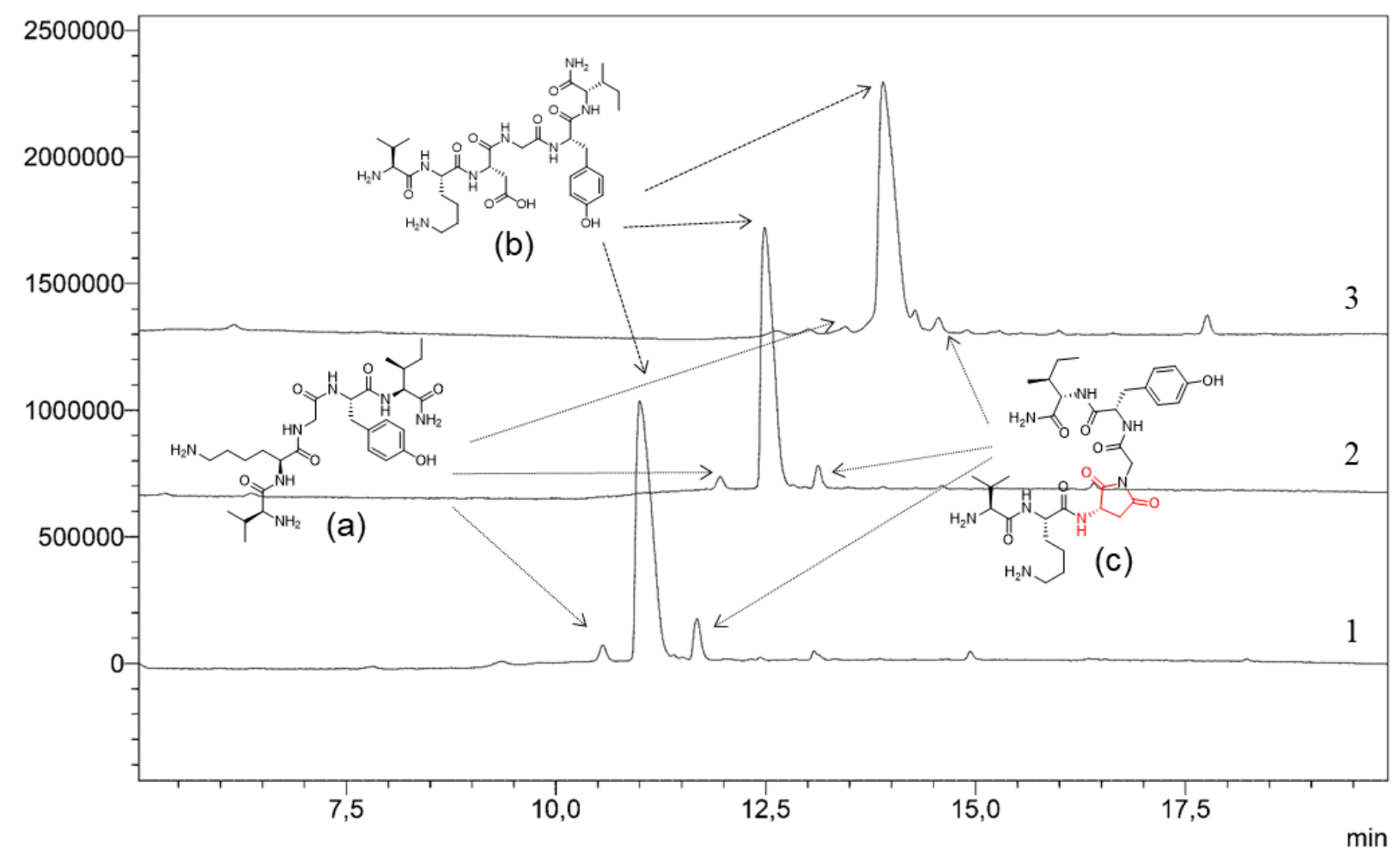

Figure S7. Comparison between chromatograms obtained for compounds 1-3. (a) des-Asp by-product $\left(t_{R}=10.5 \mathrm{~min}\right) ;(b)$ VKDGYI $\left(t_{R}=11.1 \mathrm{~min}\right) ;(c)$ aspartimide by-product $\left(t_{R}=11.7 \mathrm{~min}\right)$. 


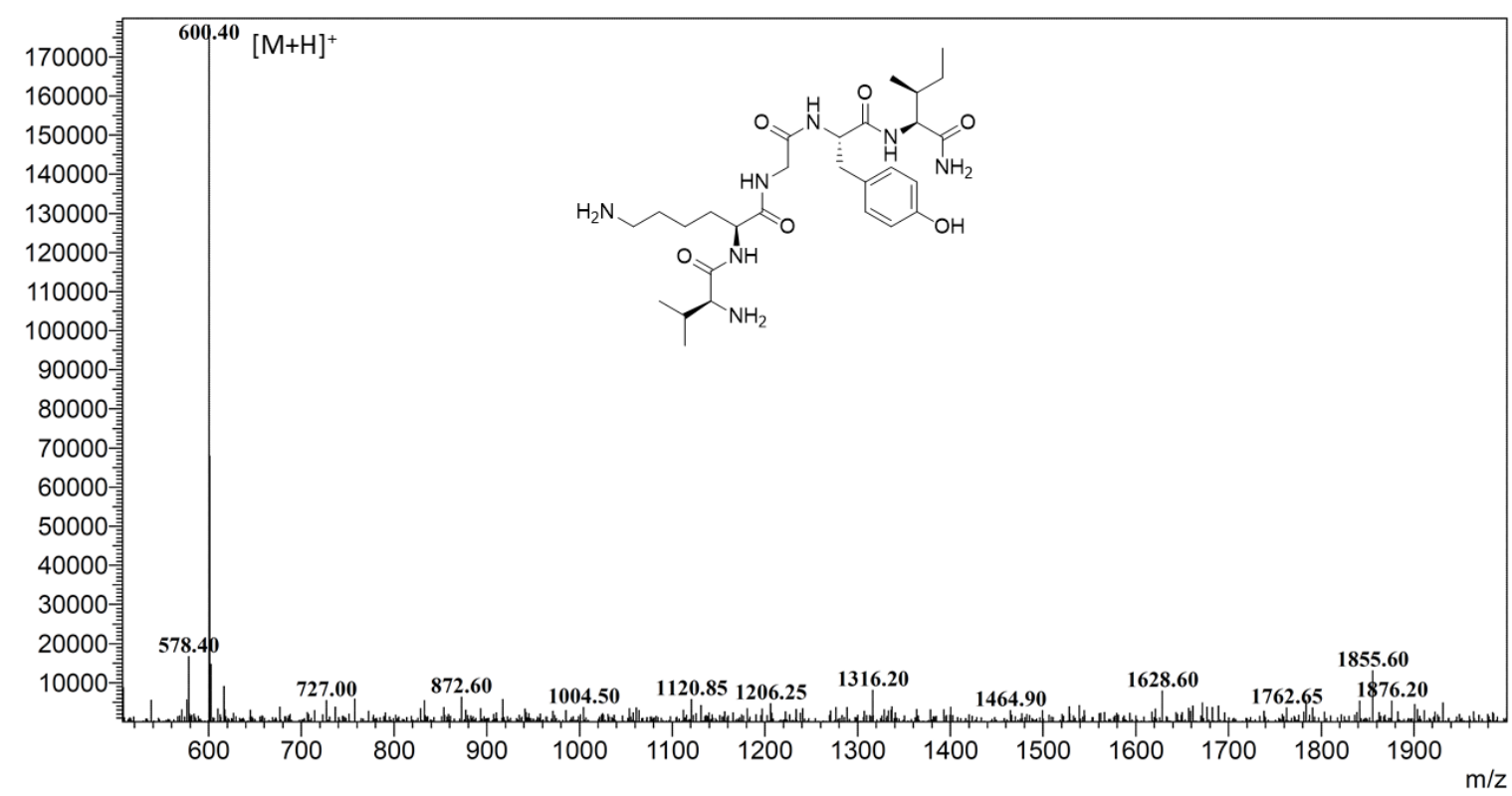

Figure S8. ESI-MS spectrum of peak at $t_{R}=10.5 \mathrm{~min}$ resulted from representative chromatograms of 13.

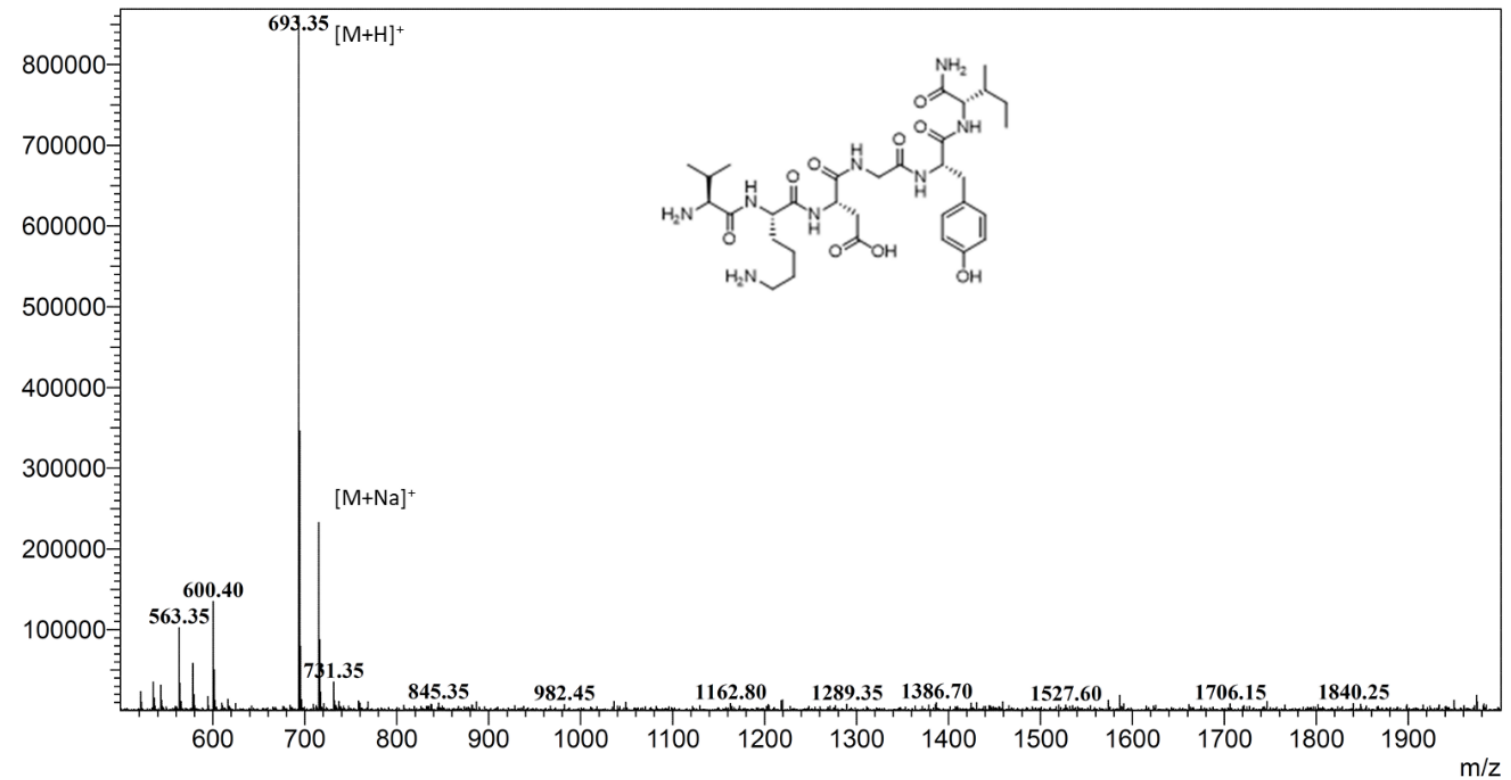

Figure S9. ESI-MS spectrum of peak at $t_{R}=11.1 \mathrm{~min}$ resulted from representative chromatograms of 13. 


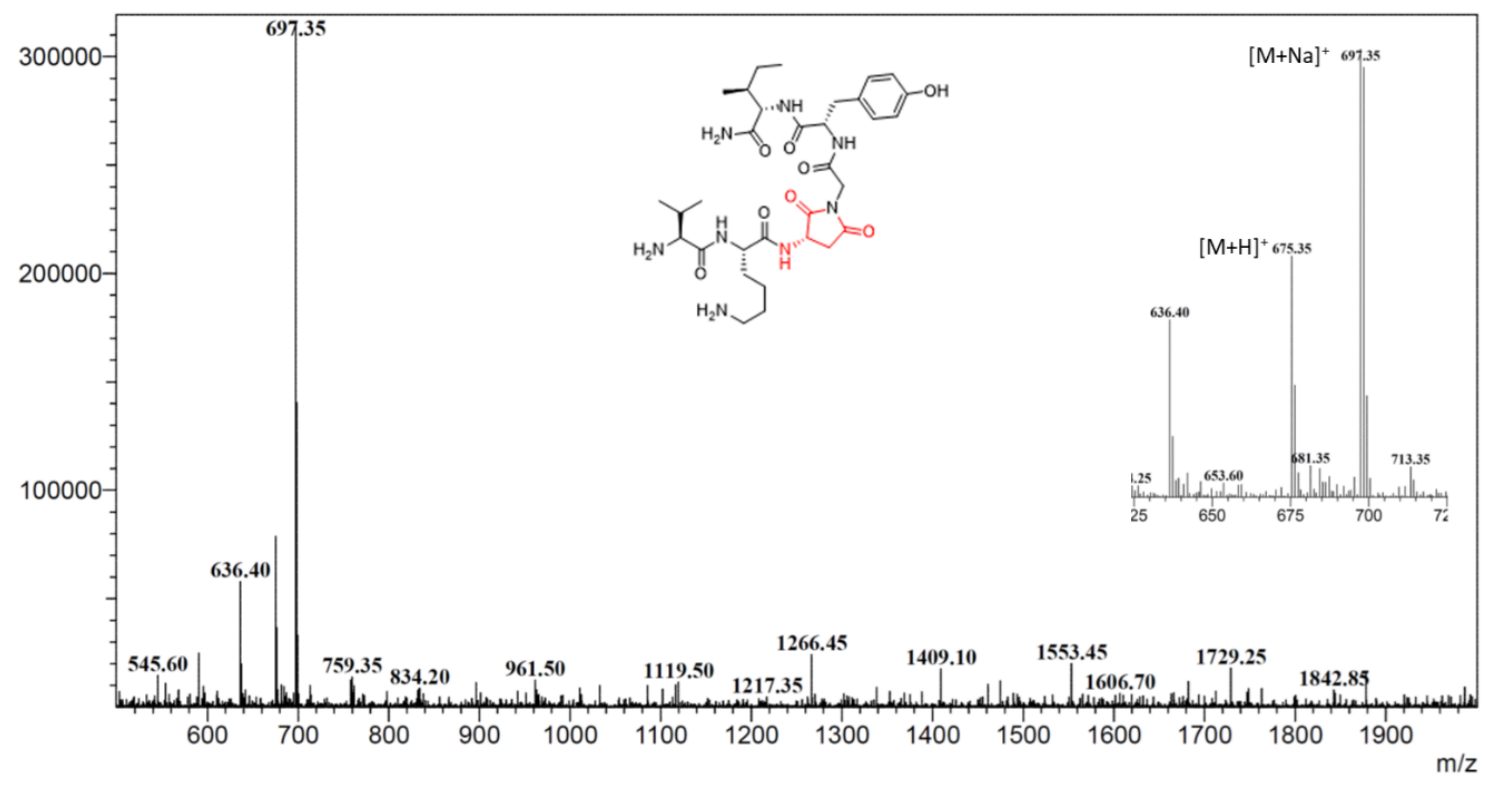

Figure S10. ESI-MS spectrum of peak at $t_{R}=11.7 \mathrm{~min}$ resulted from representative chromatograms of 1-3.

Amide formation study (Table 1, entries 1-12)

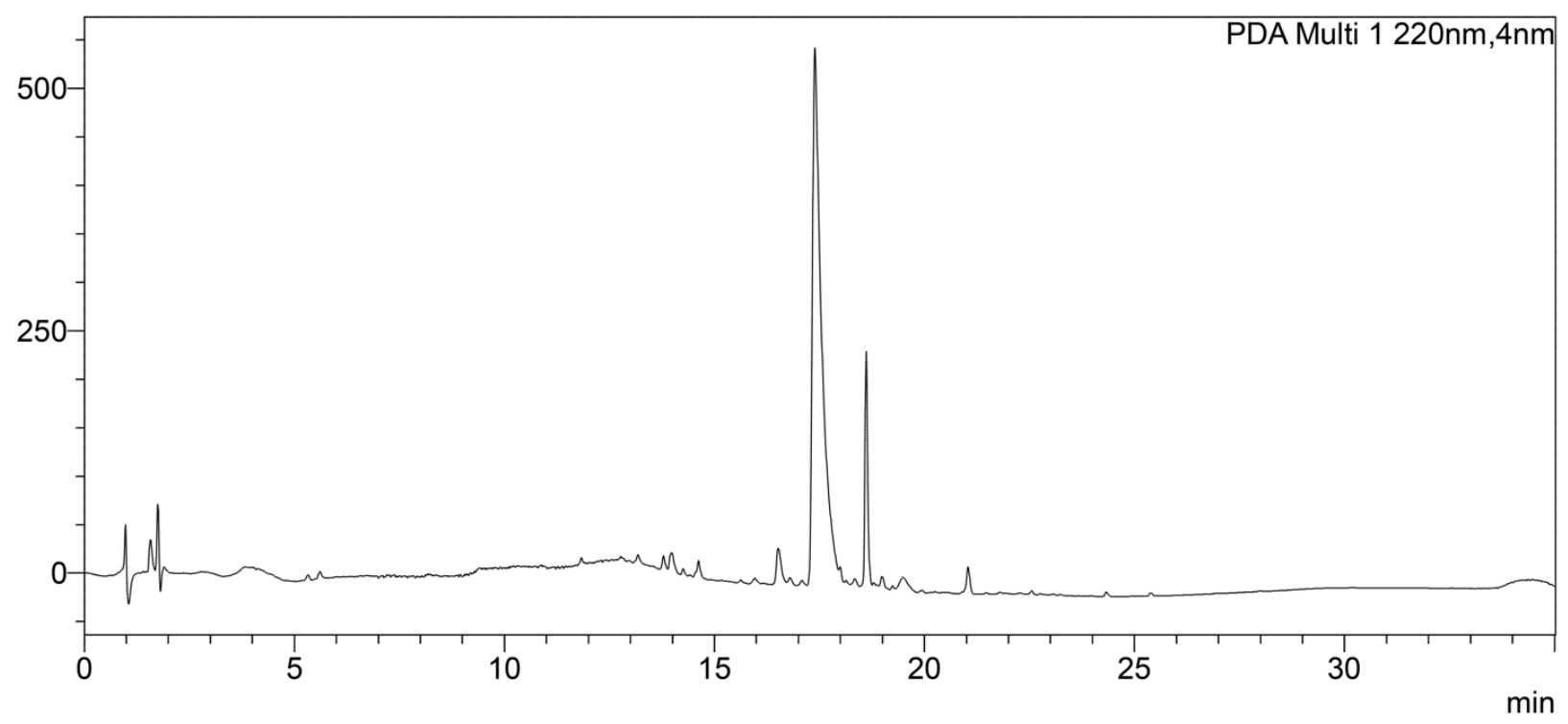

Figure S11. Representative chromatogram of entry 1 (Table 1) obtained by RP-HPLC (Shimadzu Prominance UFLC, SPD-M20A/DGU-20A3R/LC-20AD) on a Phenomenex Kinetex column (C18, 150 $\mathrm{mm} \times 4.6 \mathrm{~mm}, 5 \mu \mathrm{m}, 100 \AA$ ). Purity check/Gradient $1-\mathrm{t}_{\mathrm{R}}=17.1 \mathrm{~min}$. 


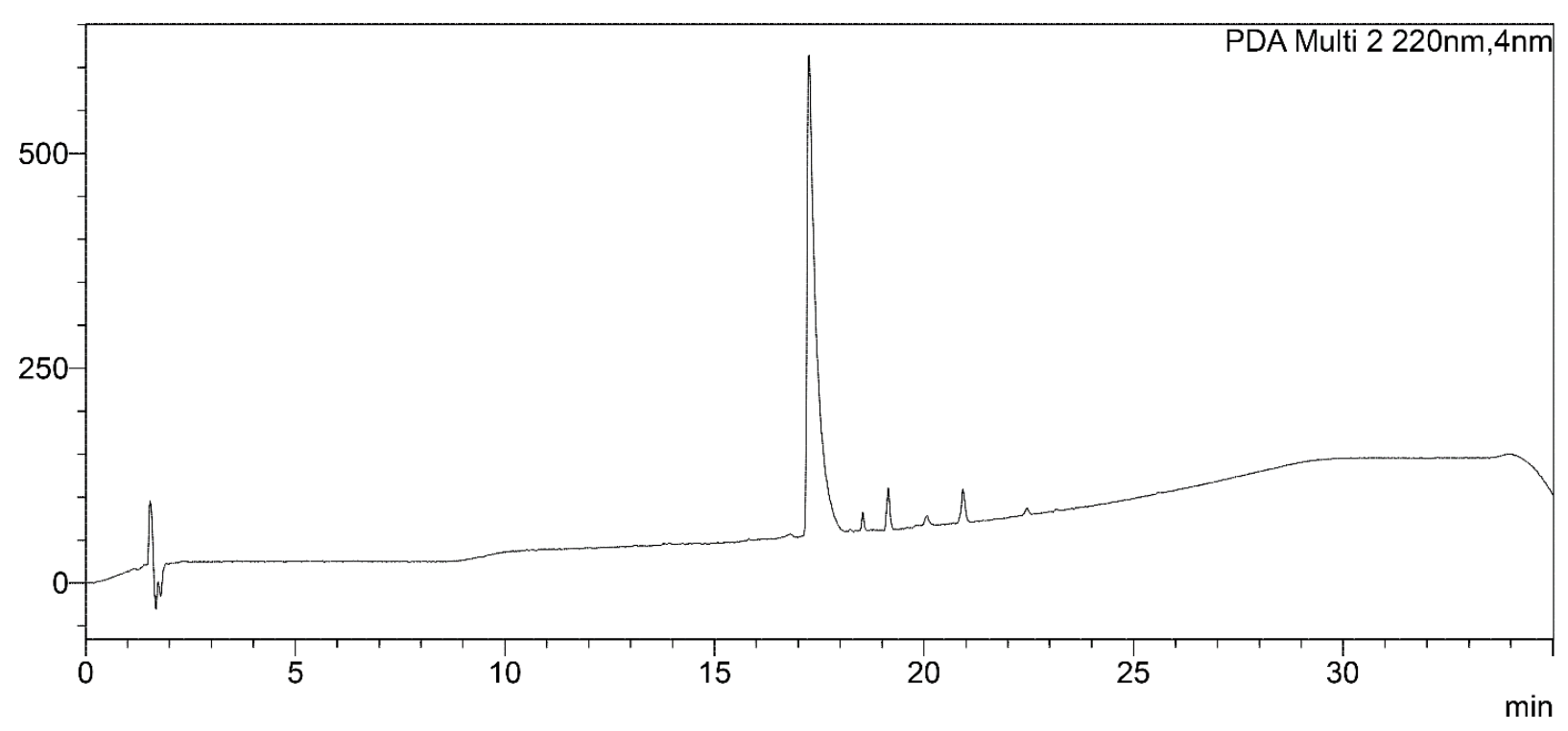

Figure S12. Representative chromatogram of entry 2 (Table 1) obtained by RP-HPLC (Shimadzu Prominance UFLC, SPD-M20A/DGU-20A3R/LC-20AD) on a Phenomenex Kinetex column (C18, 150 $\mathrm{mm} \times 4.6 \mathrm{~mm}, 5 \mu \mathrm{m}, 100 \AA$ ). Purity check/Gradient $1-\mathrm{t}_{\mathrm{R}}=17.1 \mathrm{~min}$.

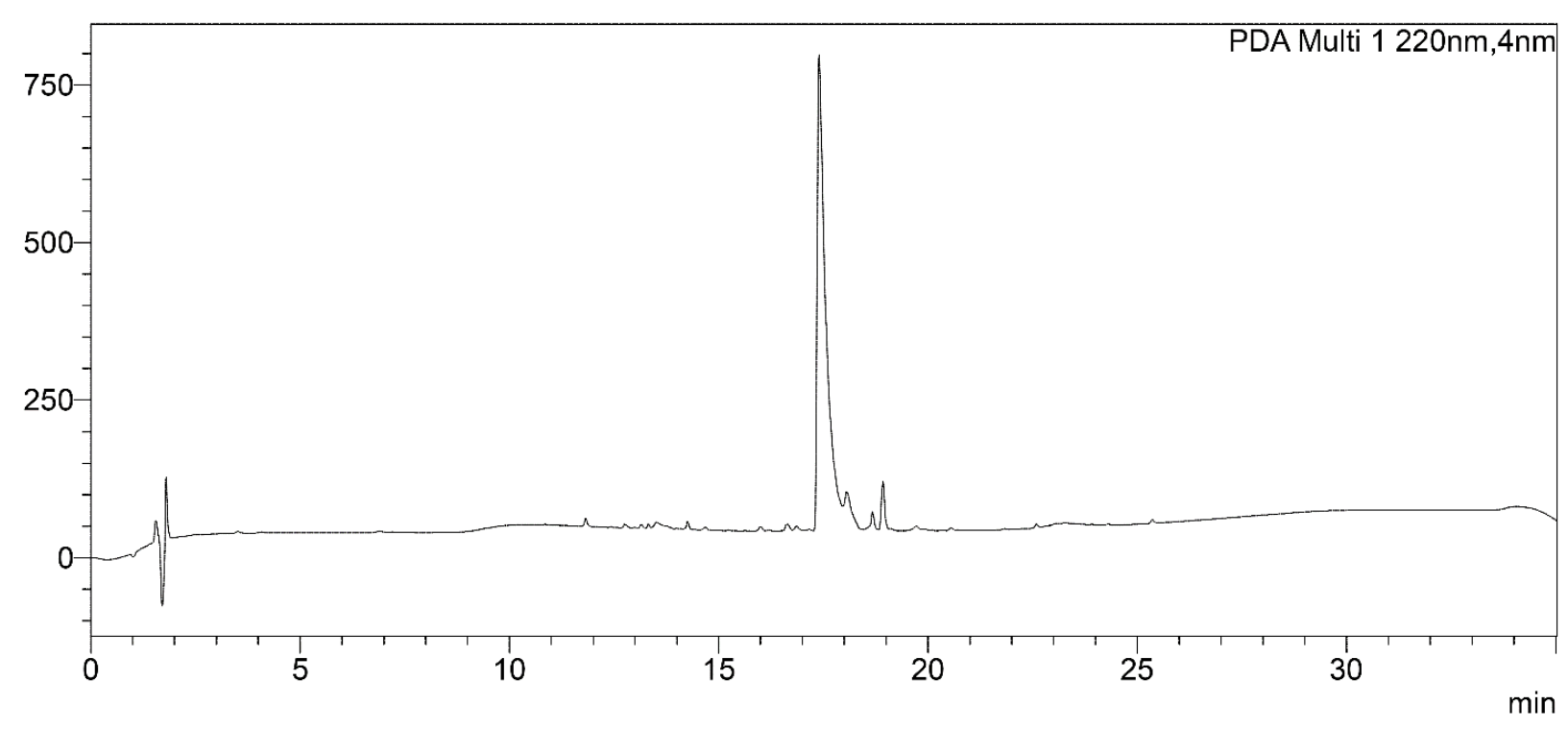

Figure S13. Representative chromatogram of entry 3 (Table 1) obtained by RP-HPLC (Shimadzu Prominance UFLC, SPD-M20A/DGU-20A3R/LC-20AD) on a Phenomenex Kinetex column (C18, 150 $\mathrm{mm} \times 4.6 \mathrm{~mm}, 5 \mu \mathrm{m}, 100 \AA$ ). Purity check/Gradient $1-\mathrm{t}_{\mathrm{R}}=17.1 \mathrm{~min}$. 


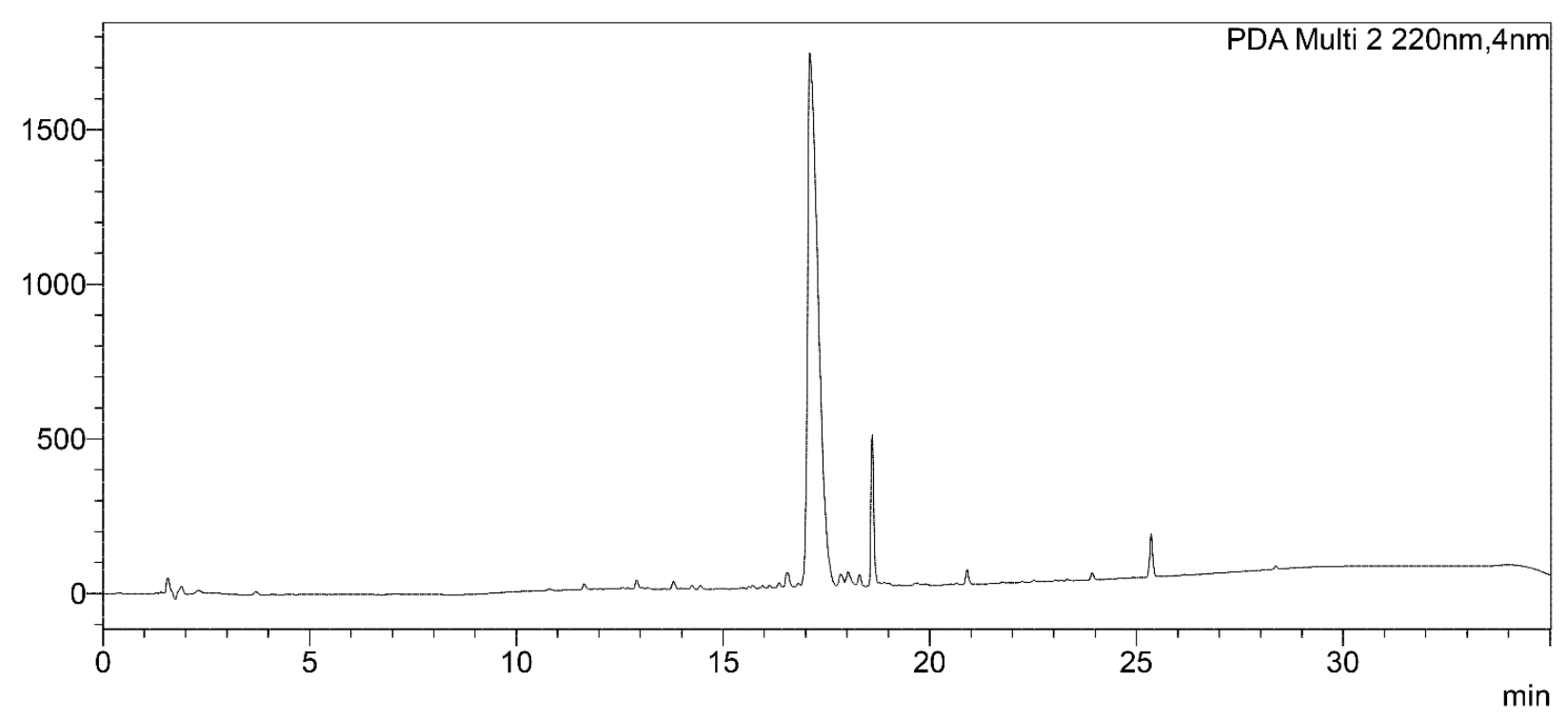

Figure S14. Representative chromatogram of entry 4 (Table 1) obtained by RP-HPLC (Shimadzu Prominance UFLC, SPD-M20A/DGU-20A3R/LC-20AD) on a Phenomenex Kinetex column (C18, 150 $\mathrm{mm} \times 4.6 \mathrm{~mm}, 5 \mu \mathrm{m}, 100 \AA$ ). Purity check/Gradient $1-\mathrm{t}_{\mathrm{R}}=17.1 \mathrm{~min}$.

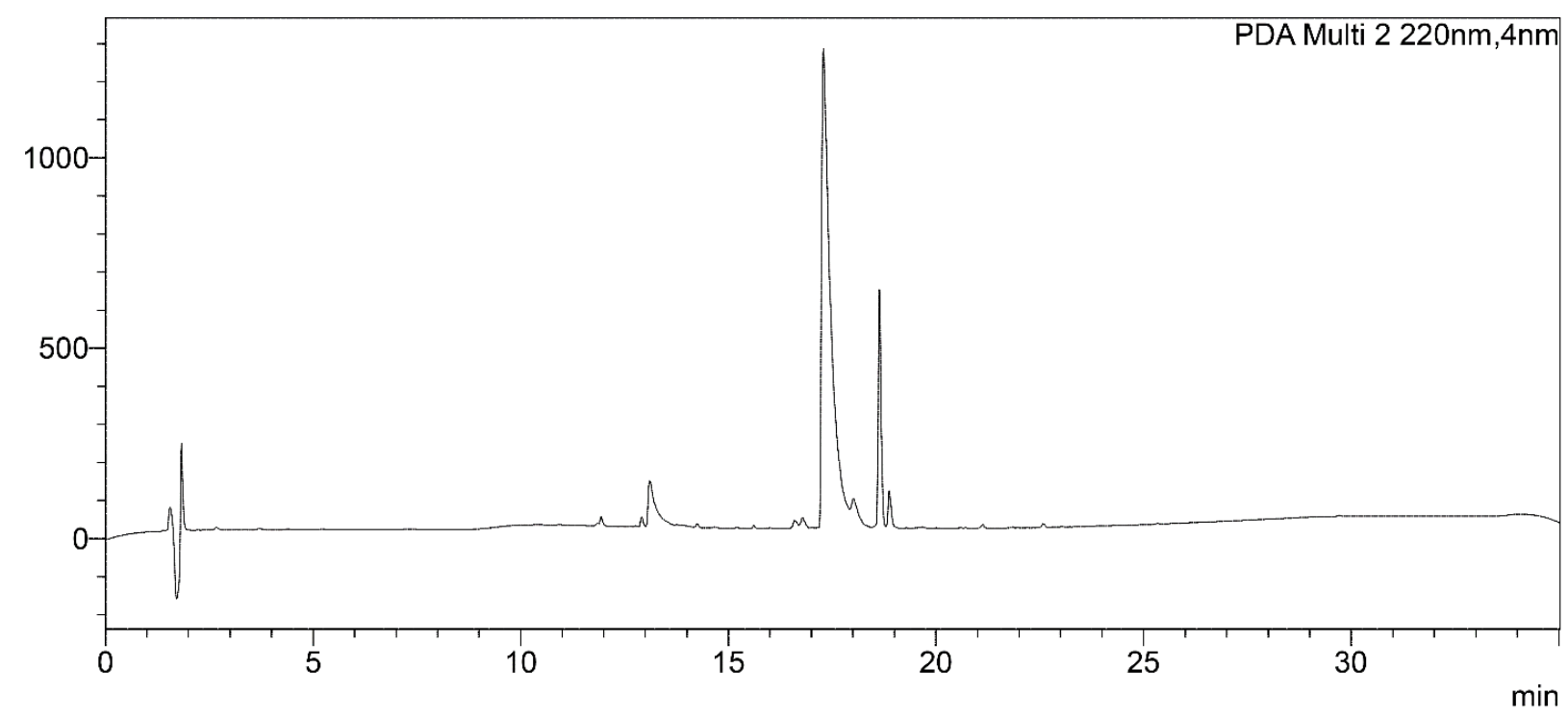

Figure S15. Representative chromatogram of entry 5 (Table 1) obtained by RP-HPLC (Shimadzu Prominance UFLC, SPD-M20A/DGU-20A3R/LC-20AD) on a Phenomenex Kinetex column (C18, 150 $\mathrm{mm} \times 4.6 \mathrm{~mm}, 5 \mu \mathrm{m}, 100 \AA$ ) . Purity check/Gradient $1-\mathrm{t}_{\mathrm{R}}=17.1 \mathrm{~min}$. 


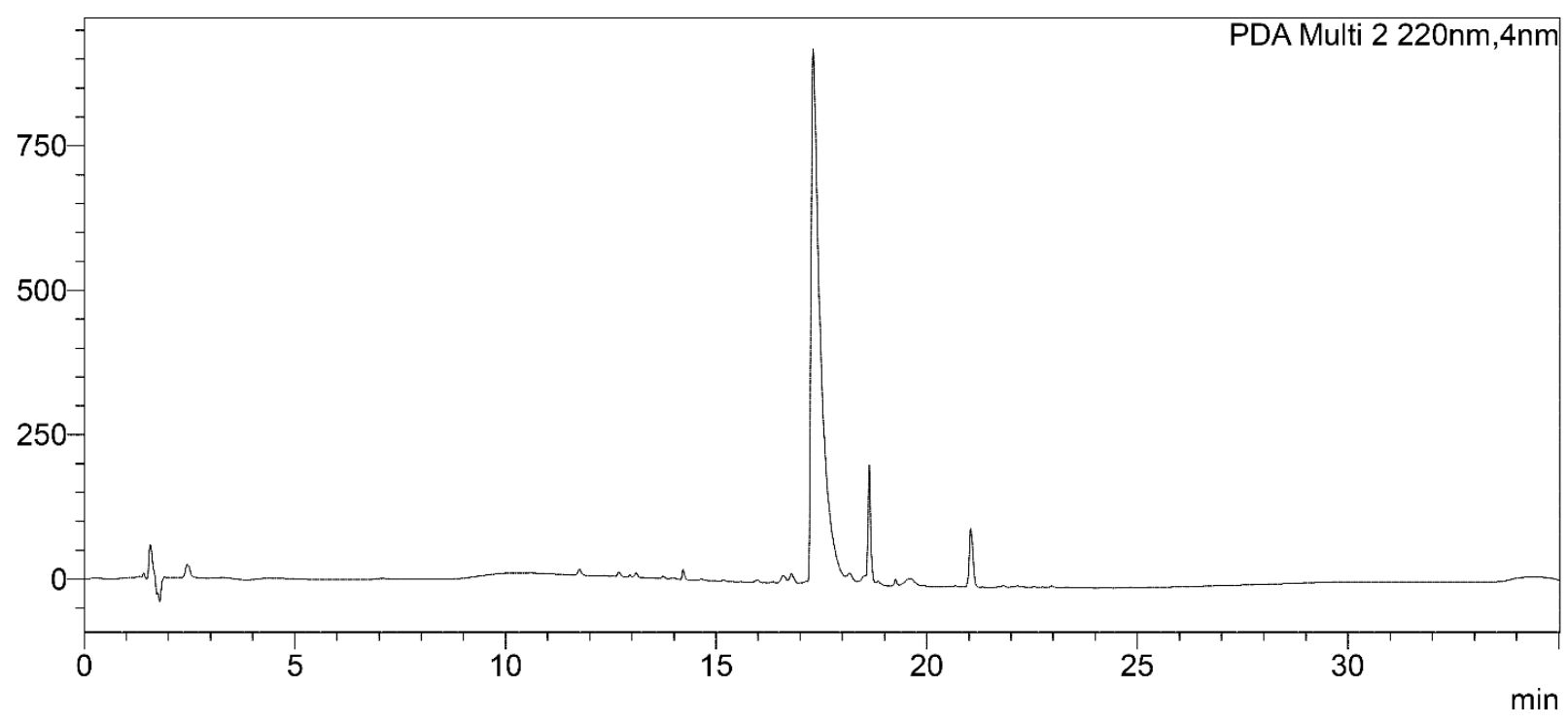

Figure S16. Representative chromatogram of entry 6 (Table 1) obtained by RP-HPLC (Shimadzu Prominance UFLC, SPD-M20A/DGU-20A3R/LC-20AD) on a Phenomenex Kinetex column (C18, 150 $\mathrm{mm} \times 4.6 \mathrm{~mm}, 5 \mu \mathrm{m}, 100 \AA$ ). Purity check/Gradient $1-\mathrm{t}_{\mathrm{R}}=17.1 \mathrm{~min}$.

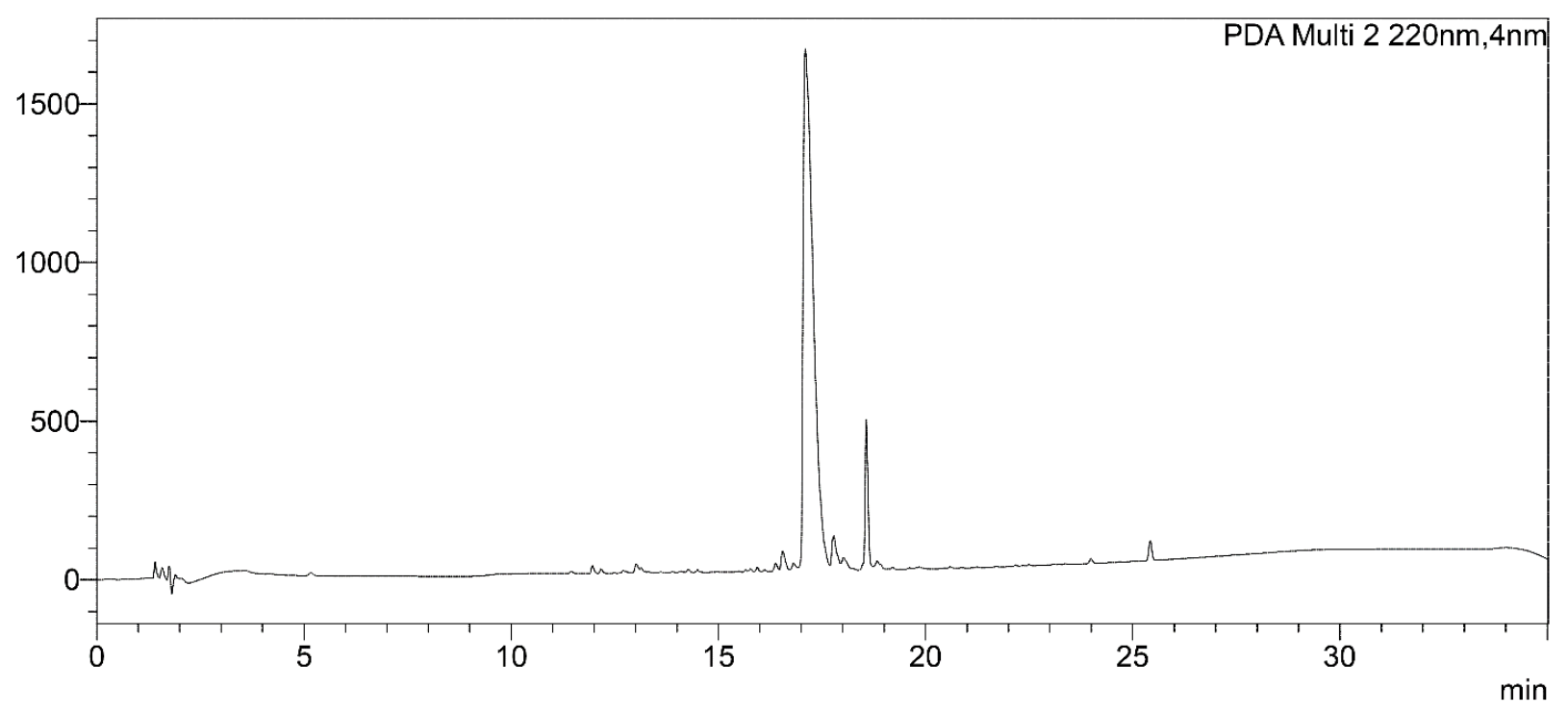

Figure S17. Representative chromatogram of entry 7 (Table 1) obtained by RP-HPLC (Shimadzu Prominance UFLC, SPD-M20A/DGU-20A3R/LC-20AD) on a Phenomenex Kinetex column (C18, 150 $\mathrm{mm} \times 4.6 \mathrm{~mm}, 5 \mu \mathrm{m}, 100 \AA$ ). Purity check/Gradient $1-\mathrm{t}_{\mathrm{R}}=17.1 \mathrm{~min}$. 


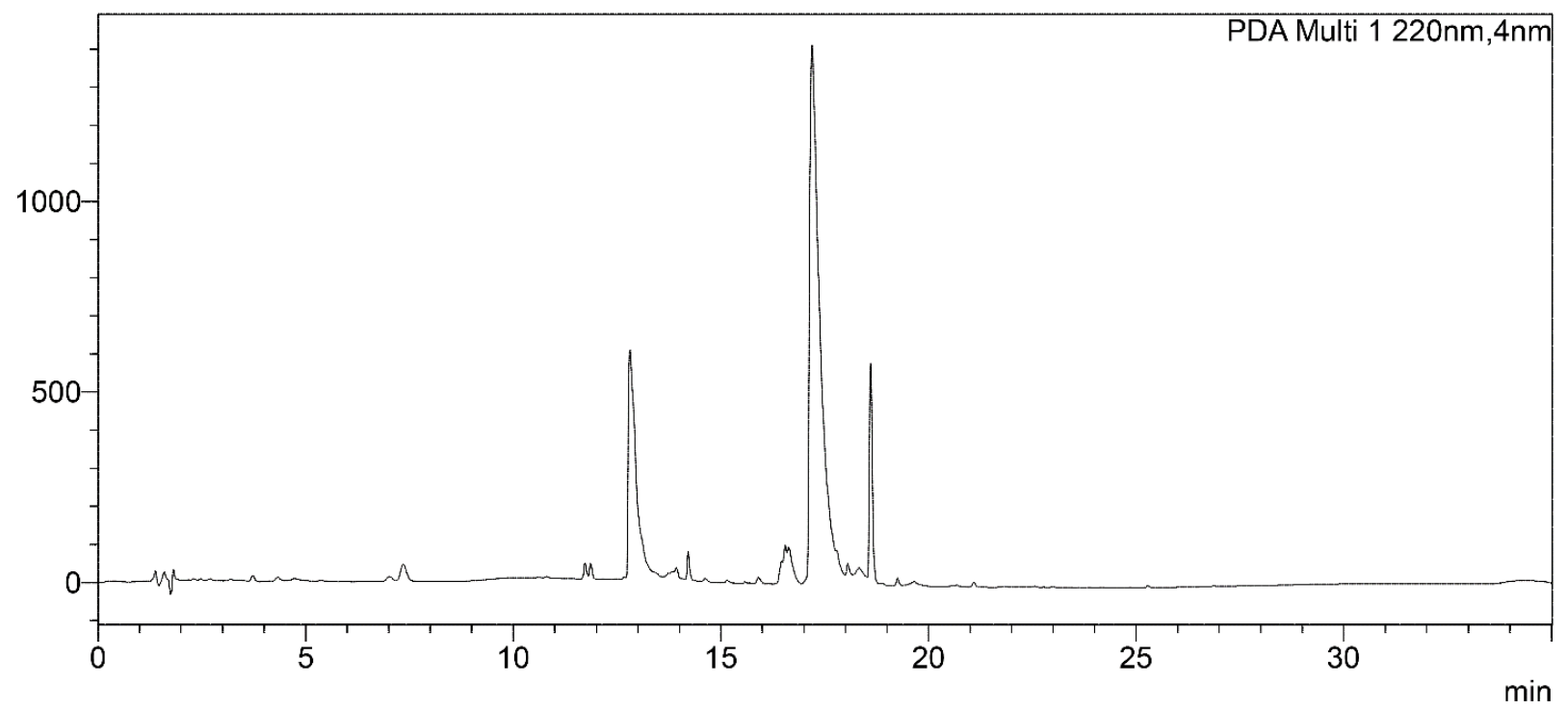

Figure S18. Representative chromatogram of entry 8 (Table 1) obtained by RP-HPLC (Shimadzu Prominance UFLC, SPD-M20A/DGU-20A3R/LC-20AD) on a Phenomenex Kinetex column (C18, 150 $\mathrm{mm} \times 4.6 \mathrm{~mm}, 5 \mu \mathrm{m}, 100 \AA$ ). Purity check/Gradient $1-\mathrm{t}_{\mathrm{R}}=17.1 \mathrm{~min}$.

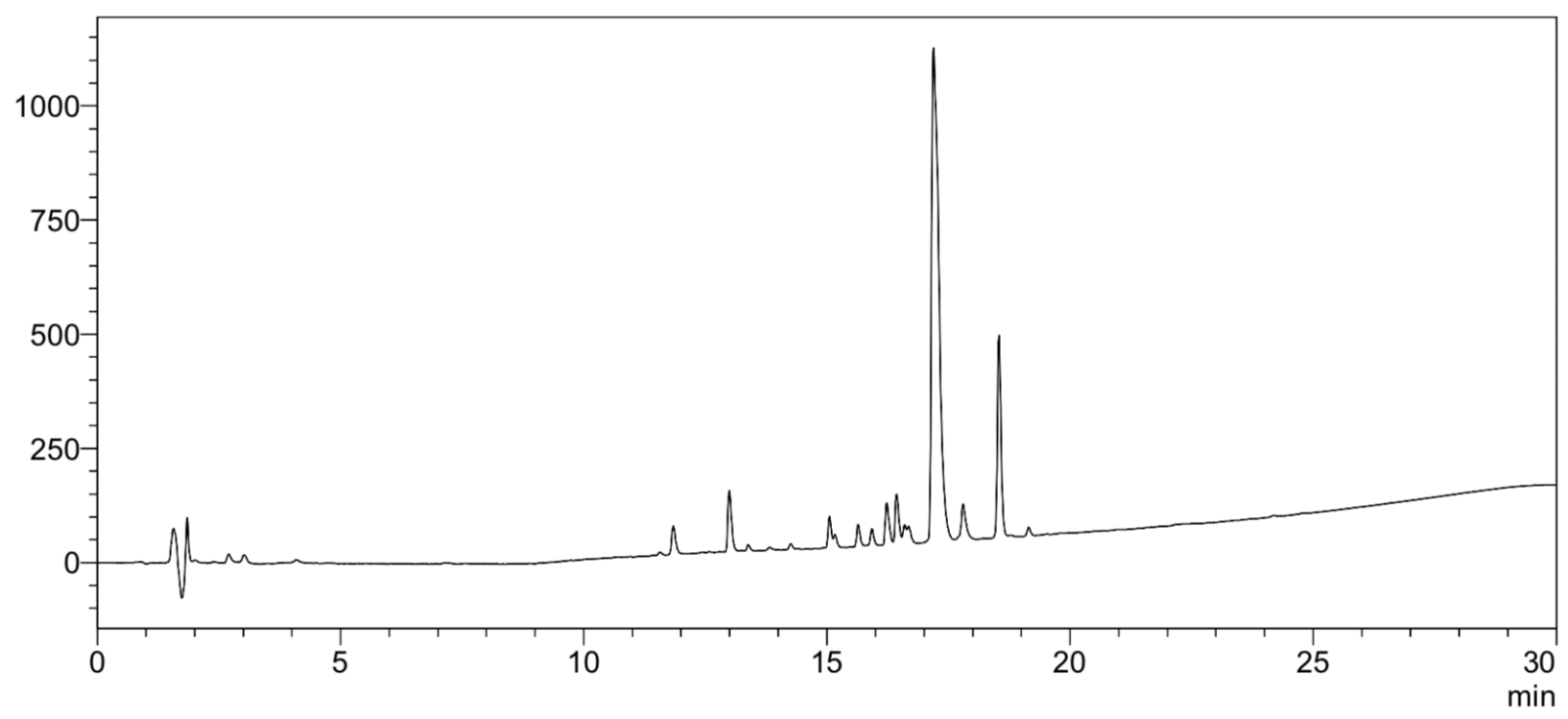

Figure S19. Representative chromatogram of entry 9 (Table 1) obtained by RP-HPLC (Shimadzu Prominance UFLC, SPD-M20A/DGU-20A3R/LC-20AD) on a Phenomenex Kinetex column (C18, 150 $\mathrm{mm} \times 4.6 \mathrm{~mm}, 5 \mu \mathrm{m}, 100 \AA$ ). Purity check/Gradient $1-\mathrm{t}_{\mathrm{R}}=17.1 \mathrm{~min}$. 


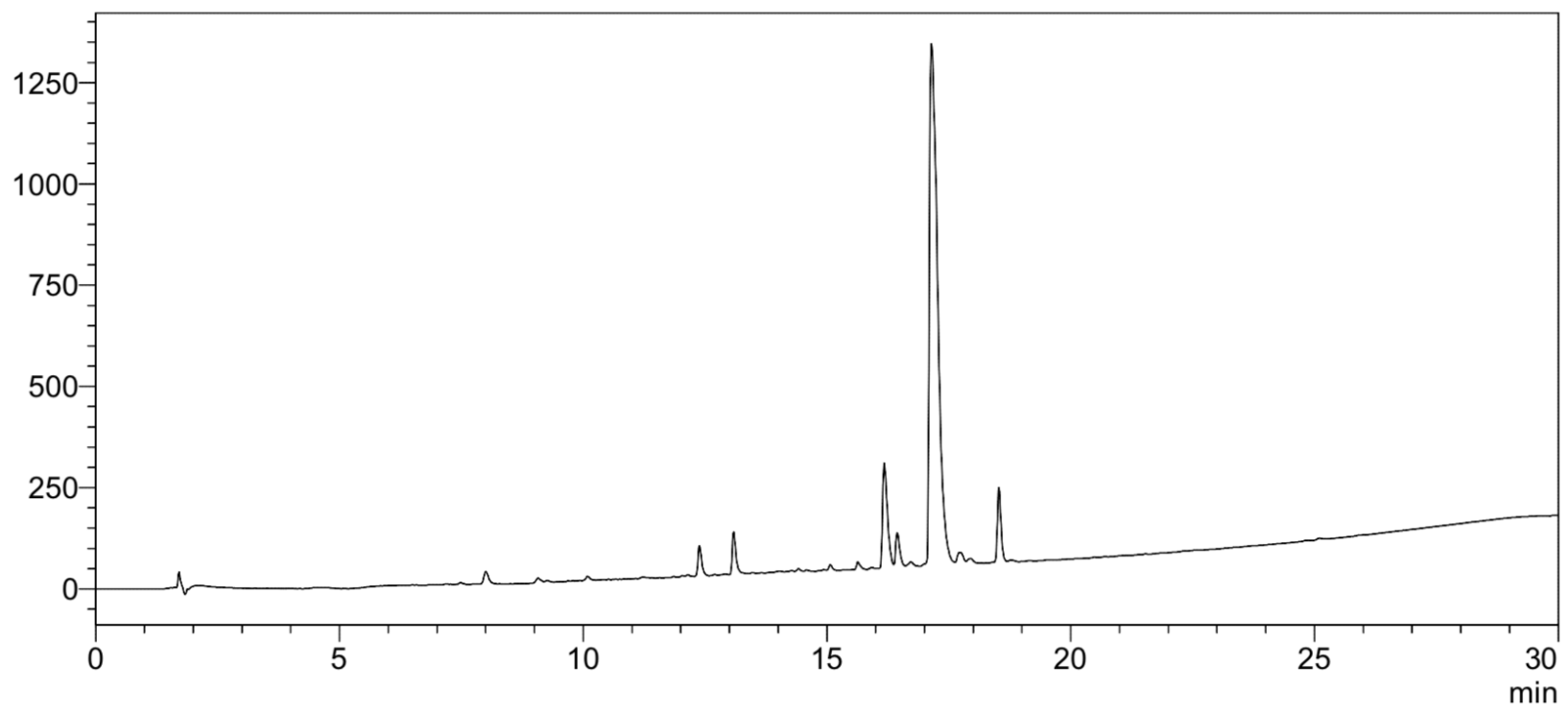

Figure S20. Representative chromatogram of entry 10 (Table 1) obtained by RP-HPLC (Shimadzu Prominance UFLC, SPD-M20A/DGU-20A3R/LC-20AD) on a Phenomenex Kinetex column (C18, 150 $\mathrm{mm} \times 4.6 \mathrm{~mm}, 5 \mu \mathrm{m}, 100 \AA$ ). Purity check/Gradient $1-\mathrm{t}_{\mathrm{R}}=17.1 \mathrm{~min}$.

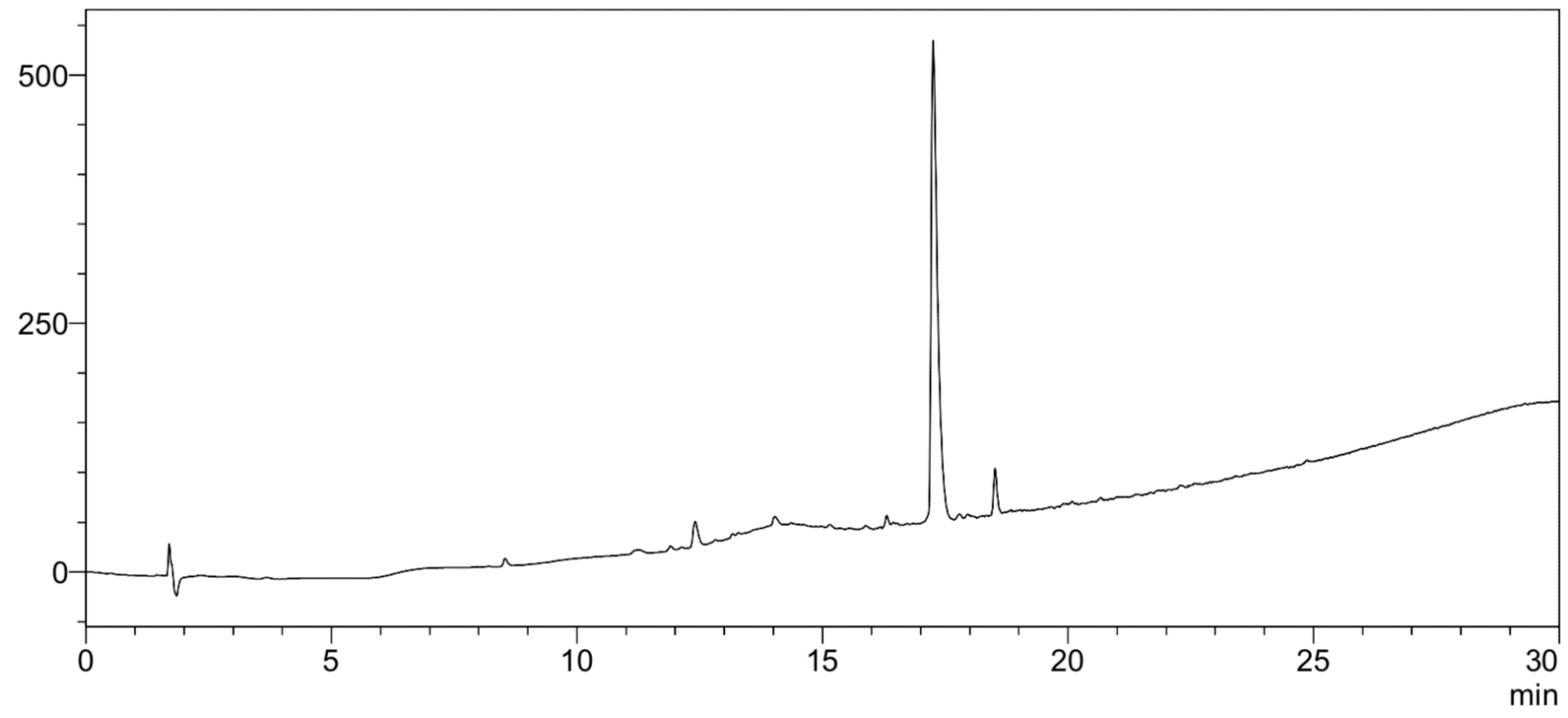

Figure S21. Representative chromatogram of entry 11 (Table 1) obtained by RP-HPLC (Shimadzu Prominance UFLC, SPD-M20A/DGU-20A3R/LC-20AD) on a Phenomenex Kinetex column (C18, 150 $\mathrm{mm} \times 4.6 \mathrm{~mm}, 5 \mu \mathrm{m}, 100 \AA$ ). Purity check/Gradient $1-\mathrm{t}_{\mathrm{R}}=17.1 \mathrm{~min}$. 


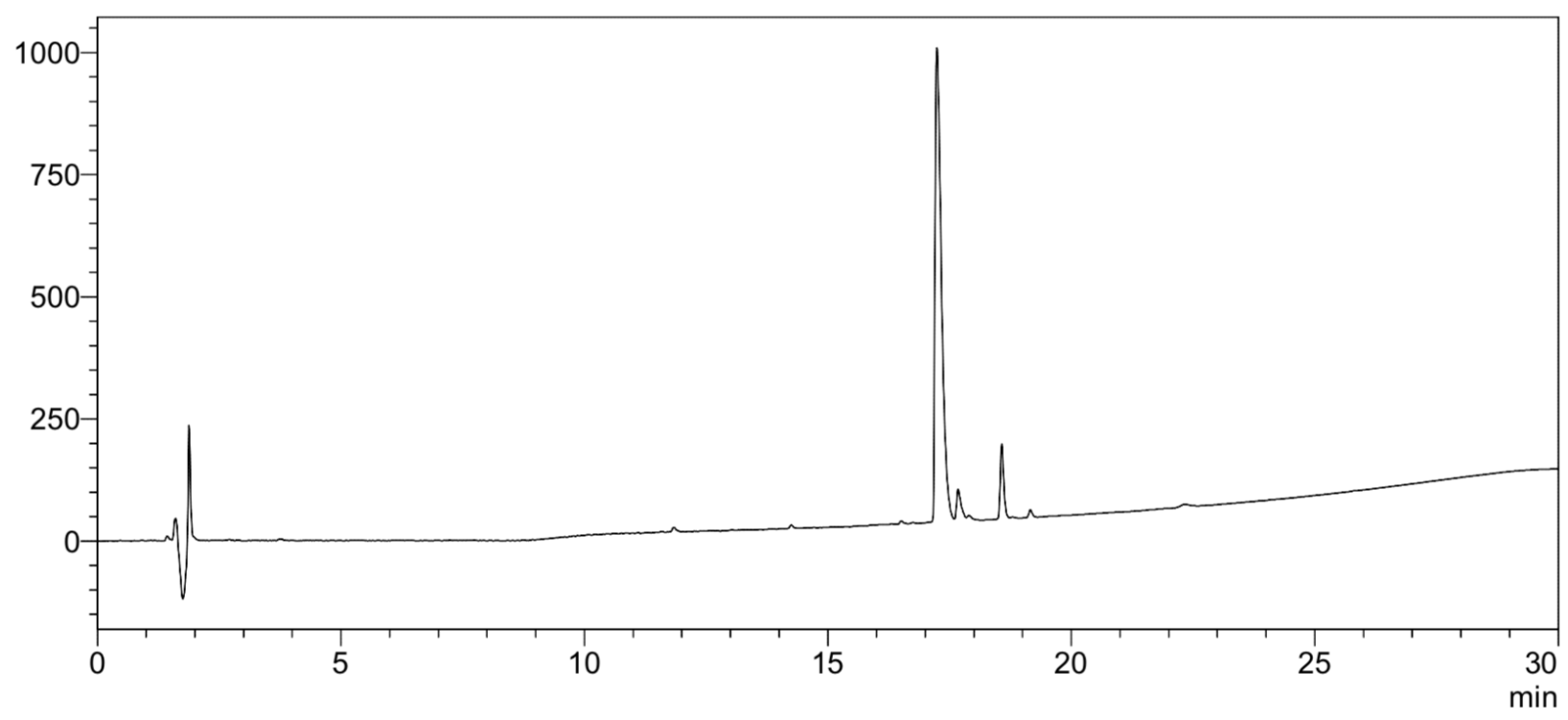

Figure S22. Representative chromatogram of entry 12 (Table 1) obtained by RP-HPLC (Shimadzu Prominance UFLC, SPD-M20A/DGU-20A3R/LC-20AD) on a Phenomenex Kinetex column (C18, 150 $\mathrm{mm} \times 4.6 \mathrm{~mm}, 5 \mu \mathrm{m}, 100 \AA)$. Purity check/Gradient $1-\mathrm{t}_{\mathrm{R}}=17.1 \mathrm{~min}$.

Coupling agents investigation (entries 7a-e)

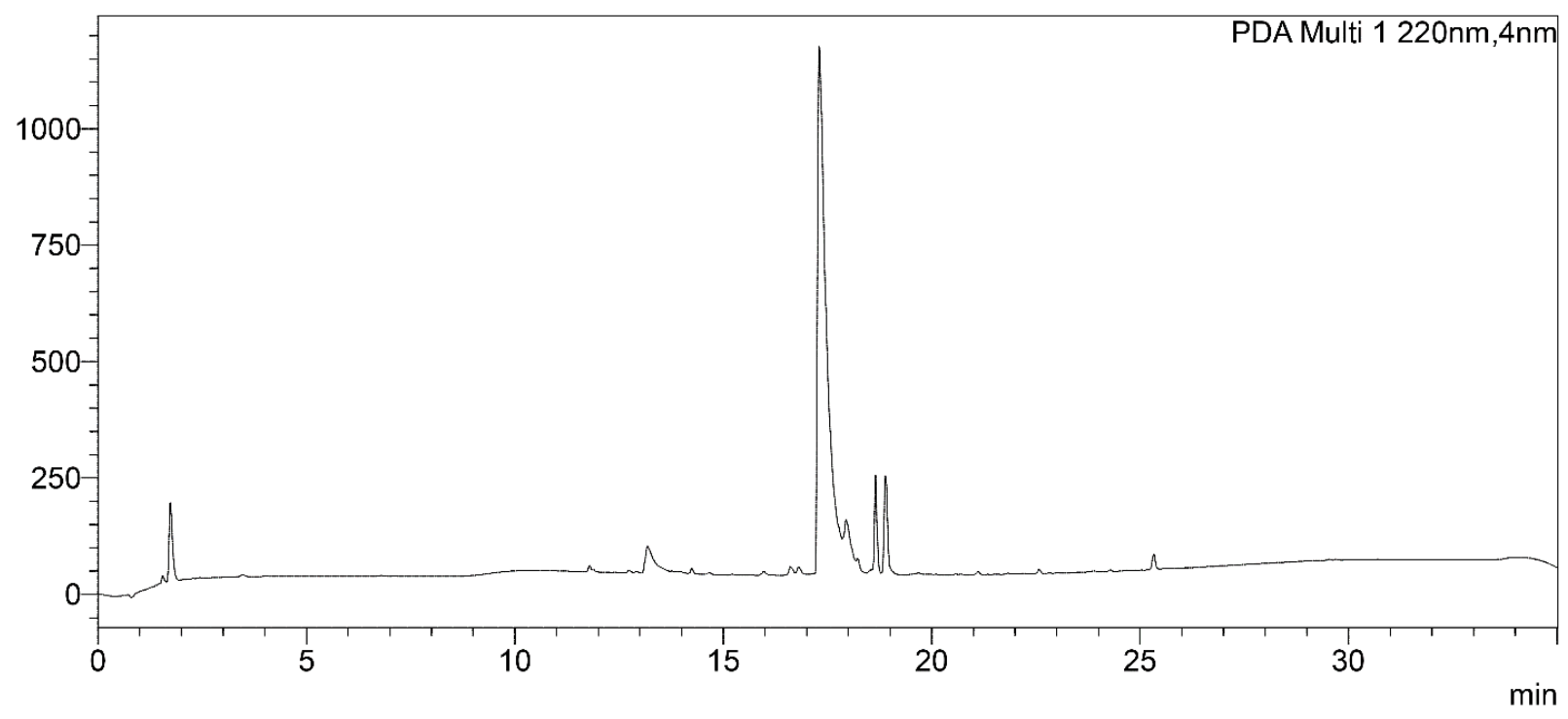

Figure S23. Representative chromatogram of entry 7a obtained by RP-HPLC (Shimadzu Prominance UFLC, SPD-M20A/DGU-20A3R/LC-20AD) on a Phenomenex Kinetex column $(\mathrm{C} 18,150 \mathrm{~mm} \times 4.6$ $\mathrm{mm}, 5 \mu \mathrm{m}, 100 \AA$ ). Purity check/Gradient $1-\mathrm{t}_{\mathrm{R}}=17.1 \mathrm{~min}$. 


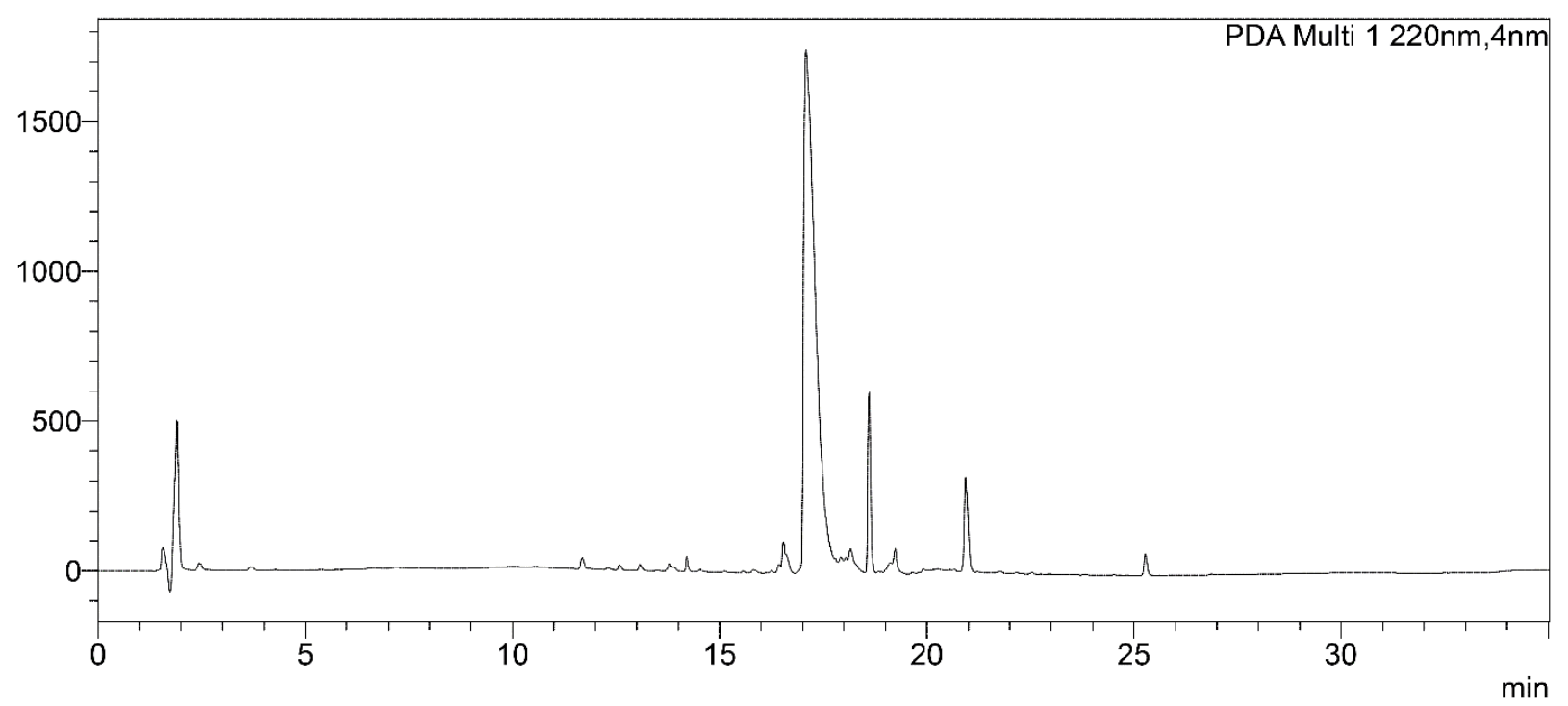

Figure S24. Representative chromatogram of entry 7b obtained by RP-HPLC (Shimadzu Prominance UFLC, SPD-M20A/DGU-20A3R/LC-20AD) on a Phenomenex Kinetex column $(\mathrm{C} 18,150 \mathrm{~mm} \times 4.6$ $\mathrm{mm}, 5 \mu \mathrm{m}, 100 \AA \AA)$. Purity check/Gradient $1-\mathrm{t}_{\mathrm{R}}=17.1 \mathrm{~min}$.

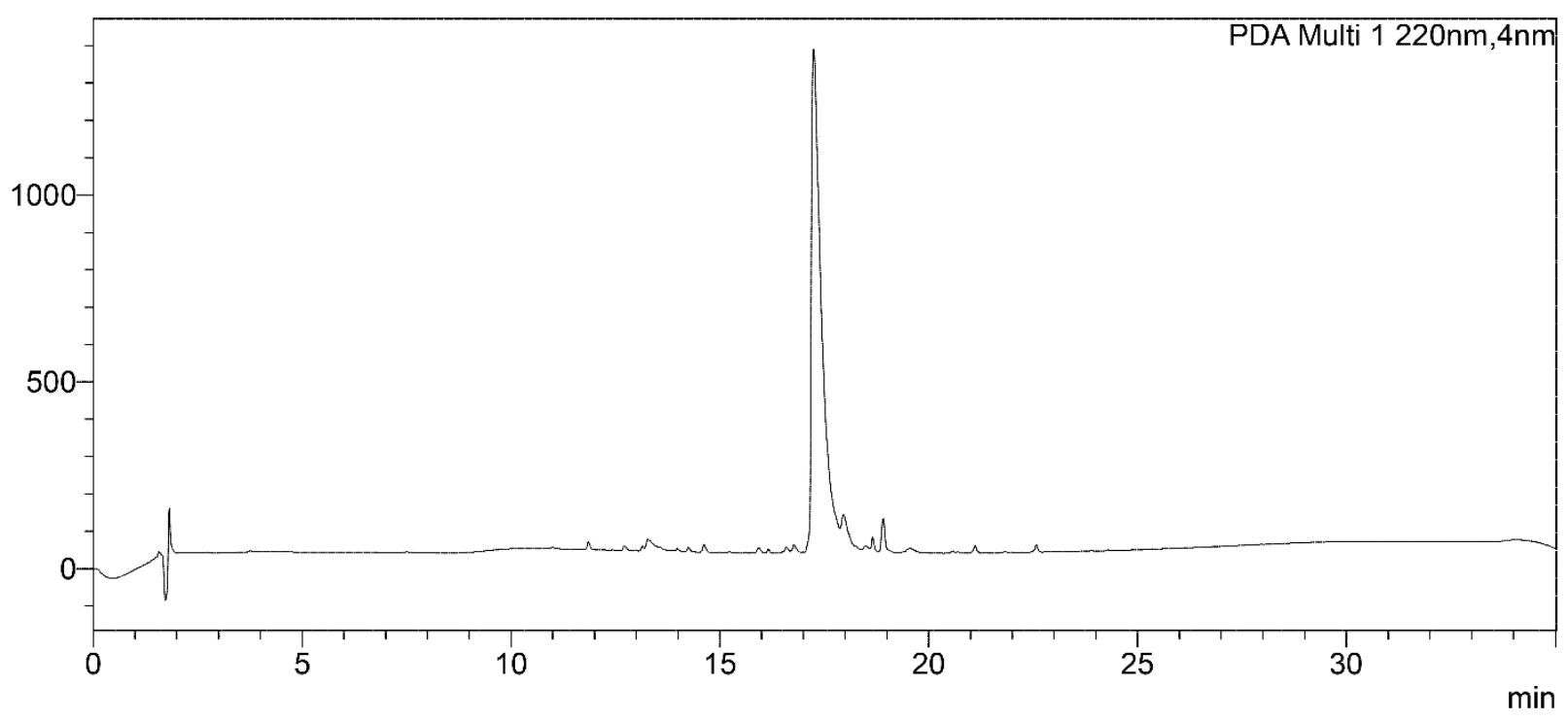

Figure S25. Representative chromatogram of entry 7c obtained by RP-HPLC (Shimadzu Prominance UFLC, SPD-M20A/DGU-20A3R/LC-20AD) on a Phenomenex Kinetex column $(\mathrm{C} 18,150 \mathrm{~mm} \times 4.6$ $\mathrm{mm}, 5 \mu \mathrm{m}, 100 \AA \AA)$. Purity check/Gradient $1-\mathrm{t}_{\mathrm{R}}=17.1 \mathrm{~min}$. 


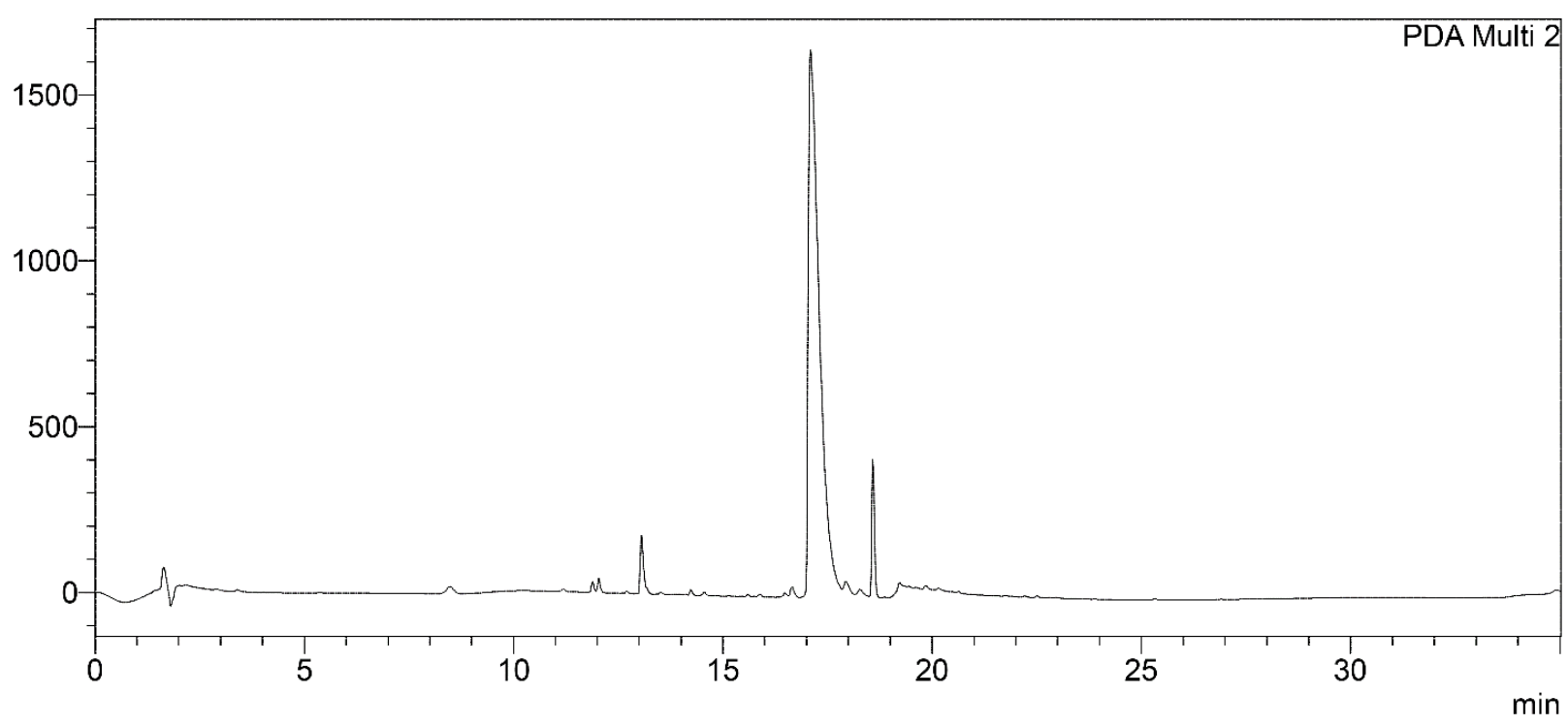

Figure S26. Representative chromatogram of entry 7d obtained by RP-HPLC (Shimadzu Prominance UFLC, SPD-M20A/DGU-20A3R/LC-20AD) on a Phenomenex Kinetex column $(\mathrm{C} 18,150 \mathrm{~mm} \times 4.6$ $\mathrm{mm}, 5 \mu \mathrm{m}, 100 \AA$ ). Purity check/Gradient $1-\mathrm{t}_{\mathrm{R}}=17.1 \mathrm{~min}$.

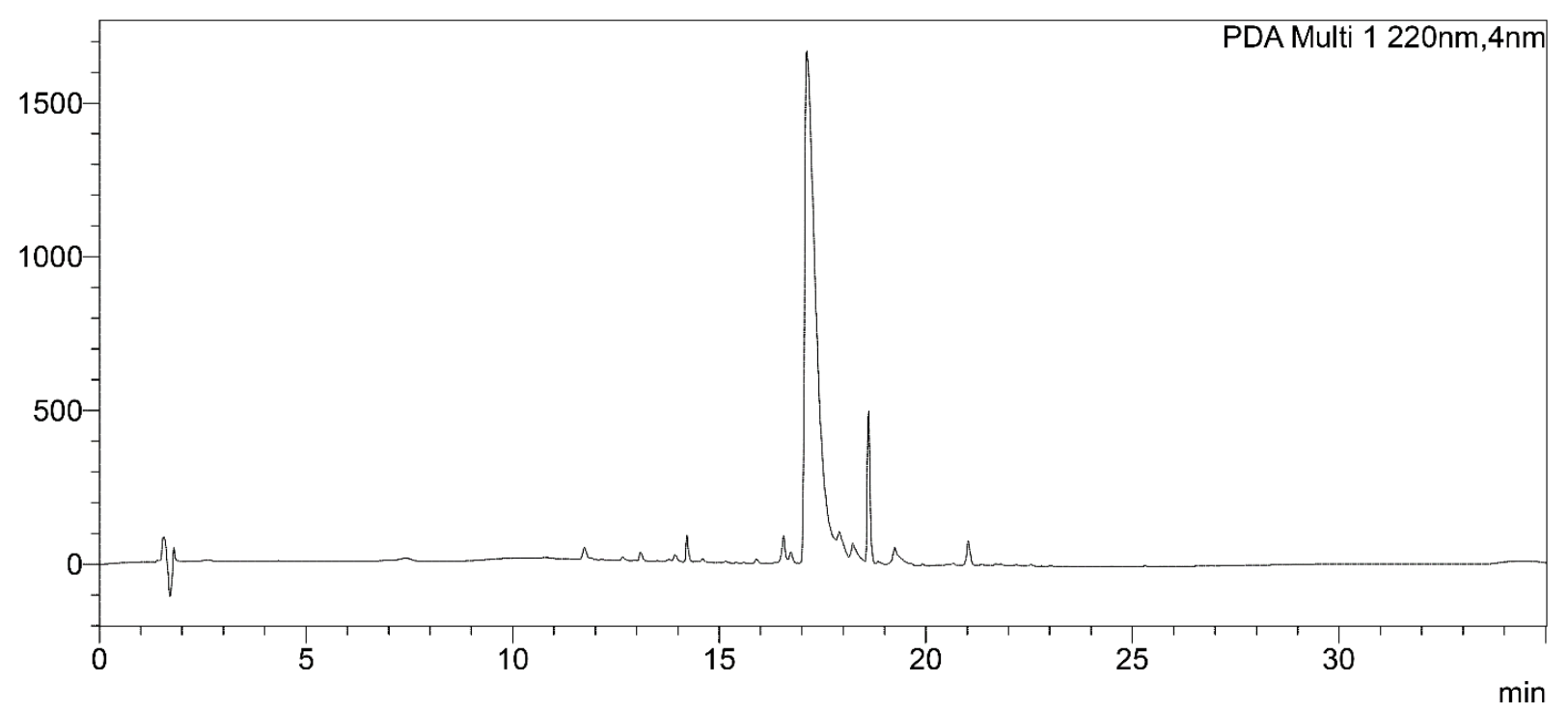

Figure S27. Representative chromatogram of enrty 7e obtained by RP-HPLC (Shimadzu Prominance UFLC, SPD-M20A/DGU-20A3R/LC-20AD) on a Phenomenex Kinetex column (C18, $150 \mathrm{~mm} \times 4.6$ $\mathrm{mm}, 5 \mu \mathrm{m}, 100 \AA$ ). Purity check/Gradient $1-\mathrm{t}_{\mathrm{R}}=17.1 \mathrm{~min}$. 


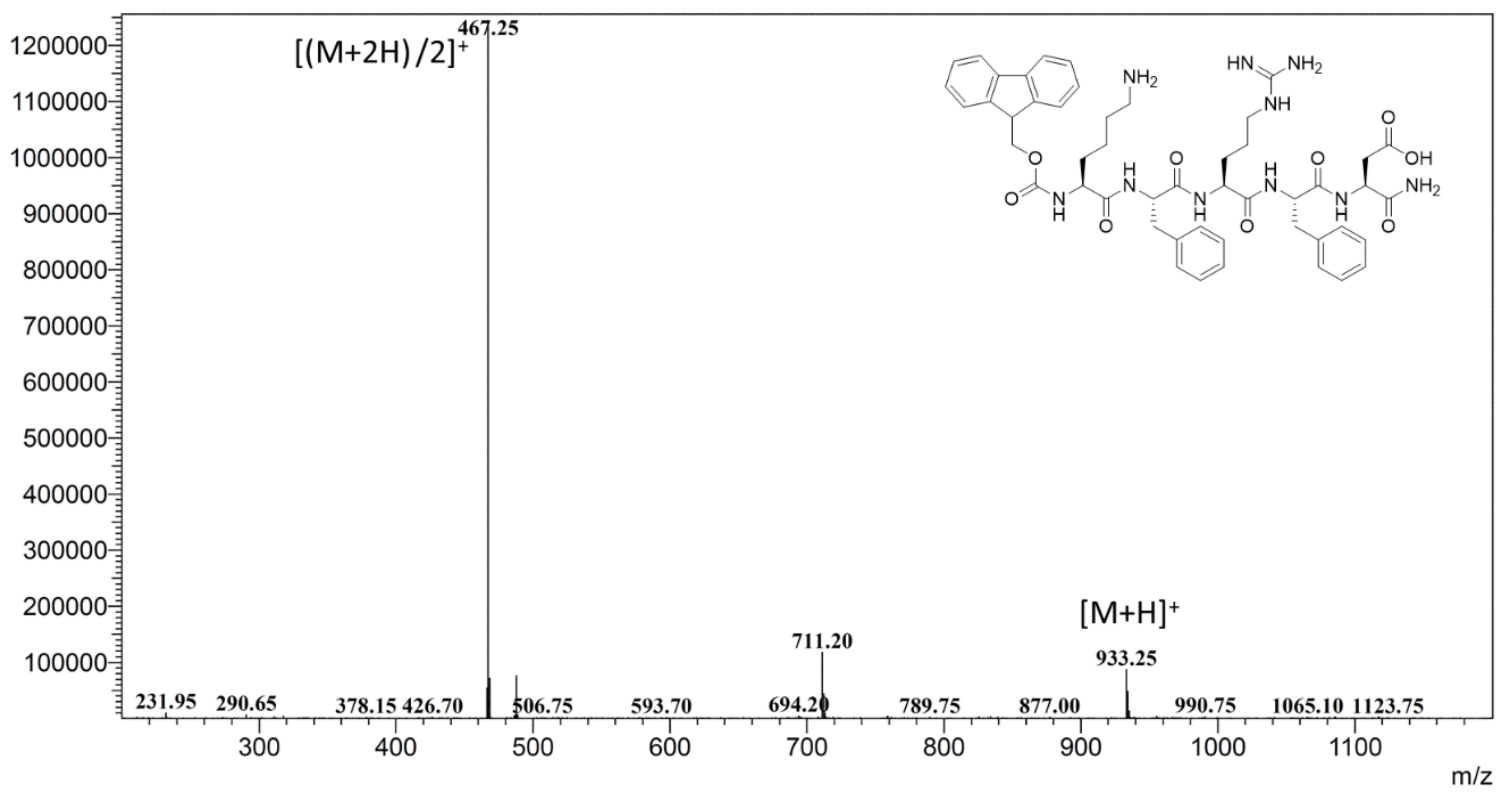

Figure S28. ESI-MS spectrum of peak at $t_{R}=17.1 \mathrm{~min}$ resulted from representative chromatograms of entries 1-12 (Table 1) and 7a-e.

Racemization study (Table 2, entries 1-8)

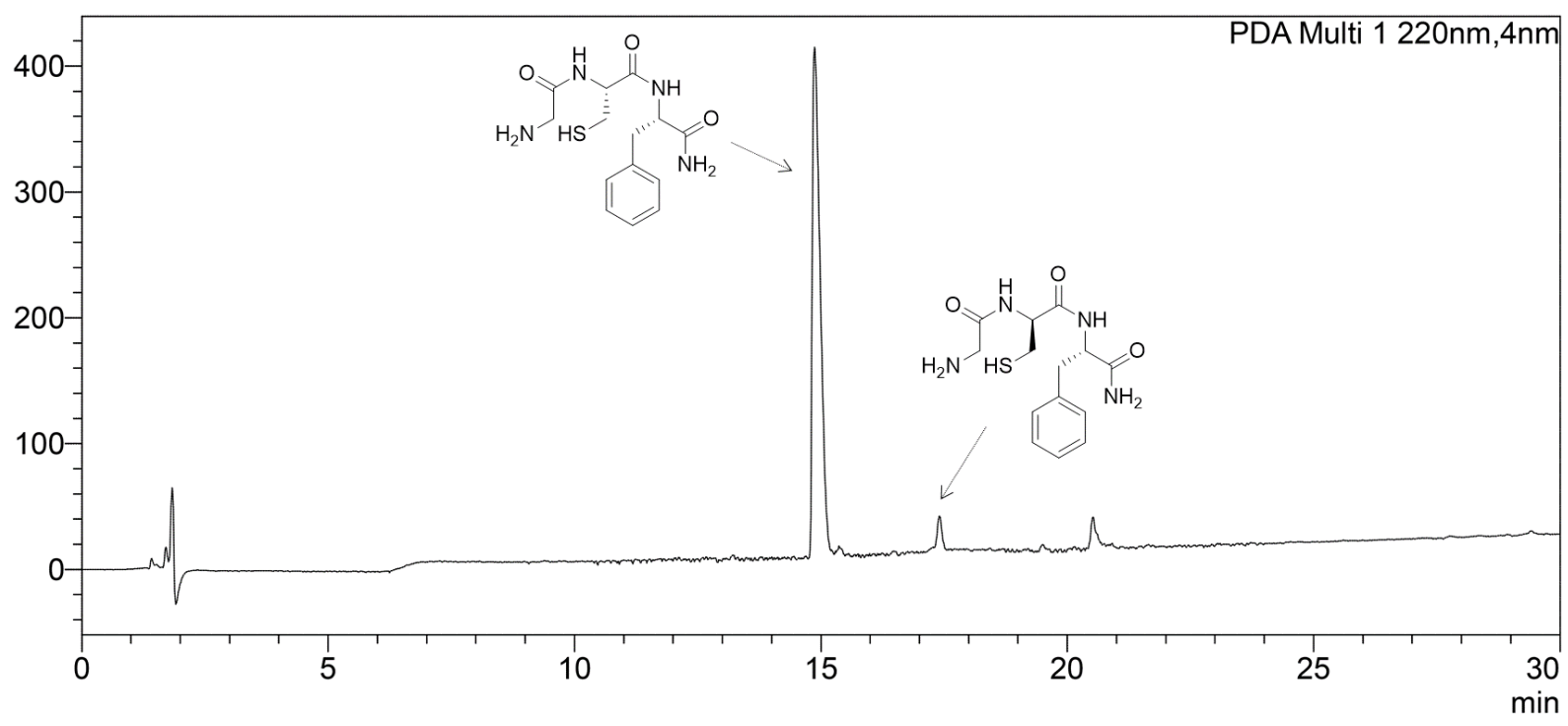

Figure S29. Chromatogram of entry 1 (Table 2) obtained by RP-HPLC (Shimadzu Prominance UFLC, SPD-M20A/DGU-20A3R/LC-20AD) on a Phenomenex Kinetex column (C18, $150 \mathrm{~mm} \times 4.6 \mathrm{~mm}, 5 \mu \mathrm{m}$, $100 \AA$ A). Purity check/Gradient $2-t_{R}=14.9$ min. 


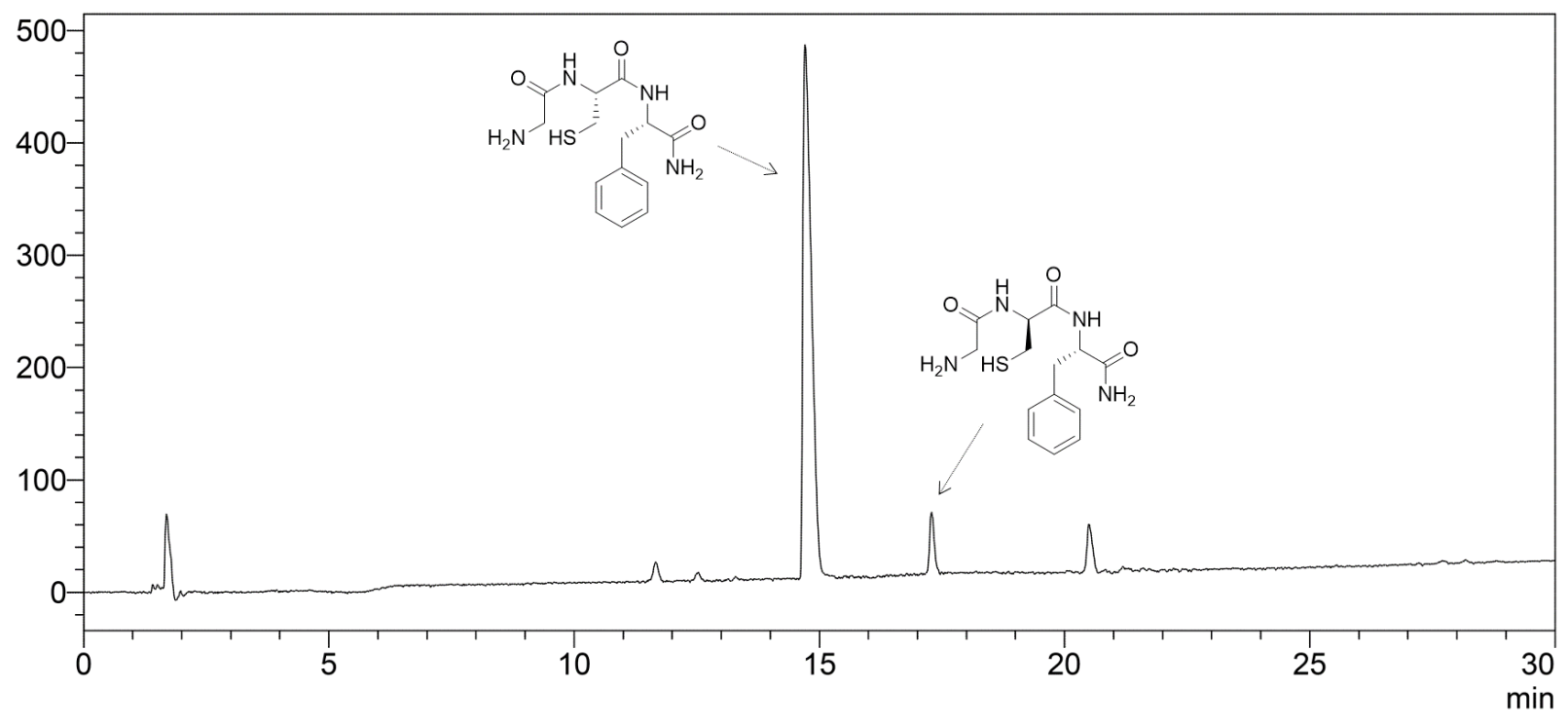

Figure S30. Chromatogram of entry 2 (Table 2) obtained by RP-HPLC (Shimadzu Prominance UFLC, SPD-M20A/DGU-20A3R/LC-20AD) on a Phenomenex Kinetex column (C18, $150 \mathrm{~mm} \times 4.6 \mathrm{~mm}, 5 \mu \mathrm{m}$, $100 \AA$ A). Purity check/Gradient $2-\mathrm{t}_{\mathrm{R}}=14.9 \mathrm{~min}$.

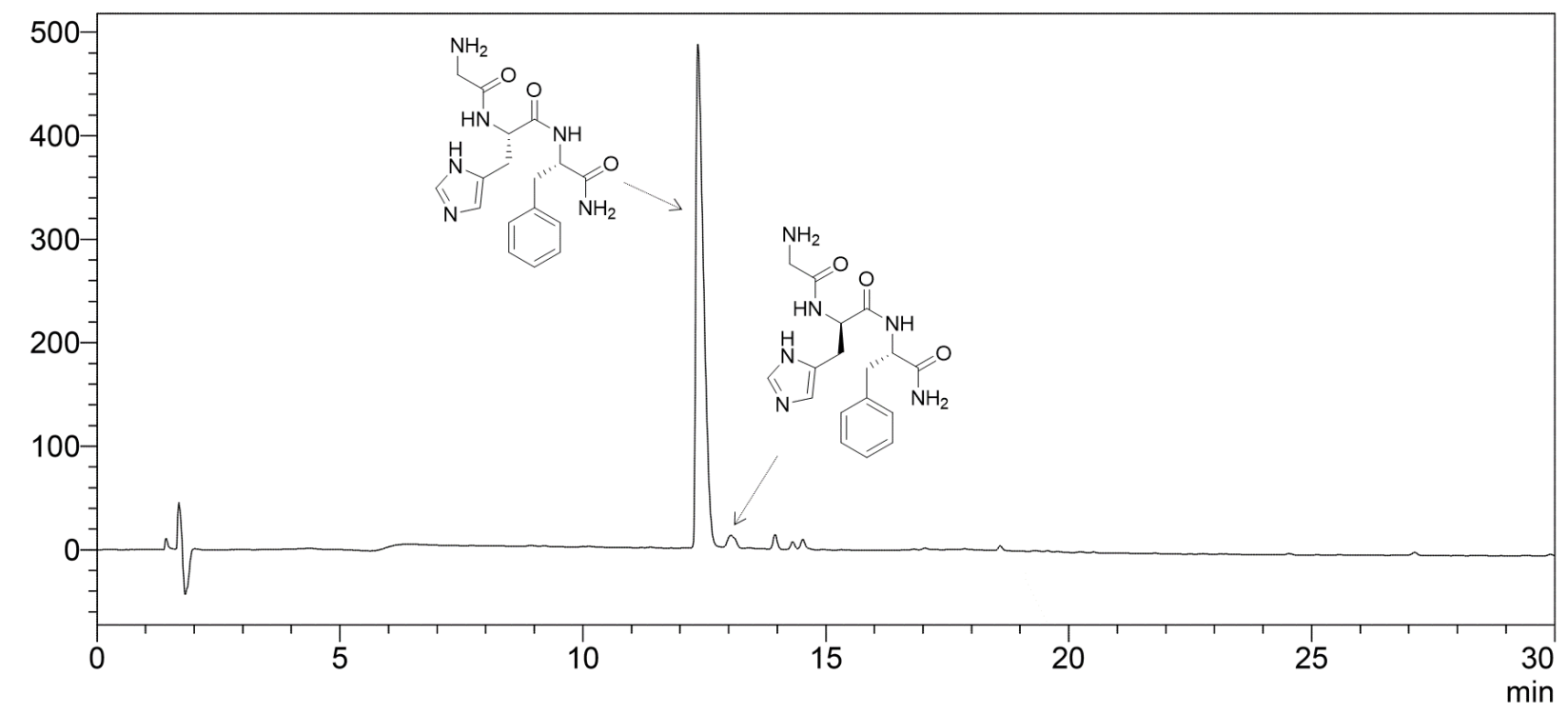

Figure S31. Chromatogram of entry 3 (Table 2) obtained by RP-HPLC (Shimadzu Prominance UFLC, SPD-M20A/DGU-20A3R/LC-20AD) on a Phenomenex Kinetex column $(\mathrm{C} 18,150 \mathrm{~mm} \times 4.6 \mathrm{~mm}, 5 \mu \mathrm{m}$, $100 \AA$ ). Purity check/Gradient $2-\mathrm{t}_{\mathrm{R}}=12.4 \mathrm{~min}$. 


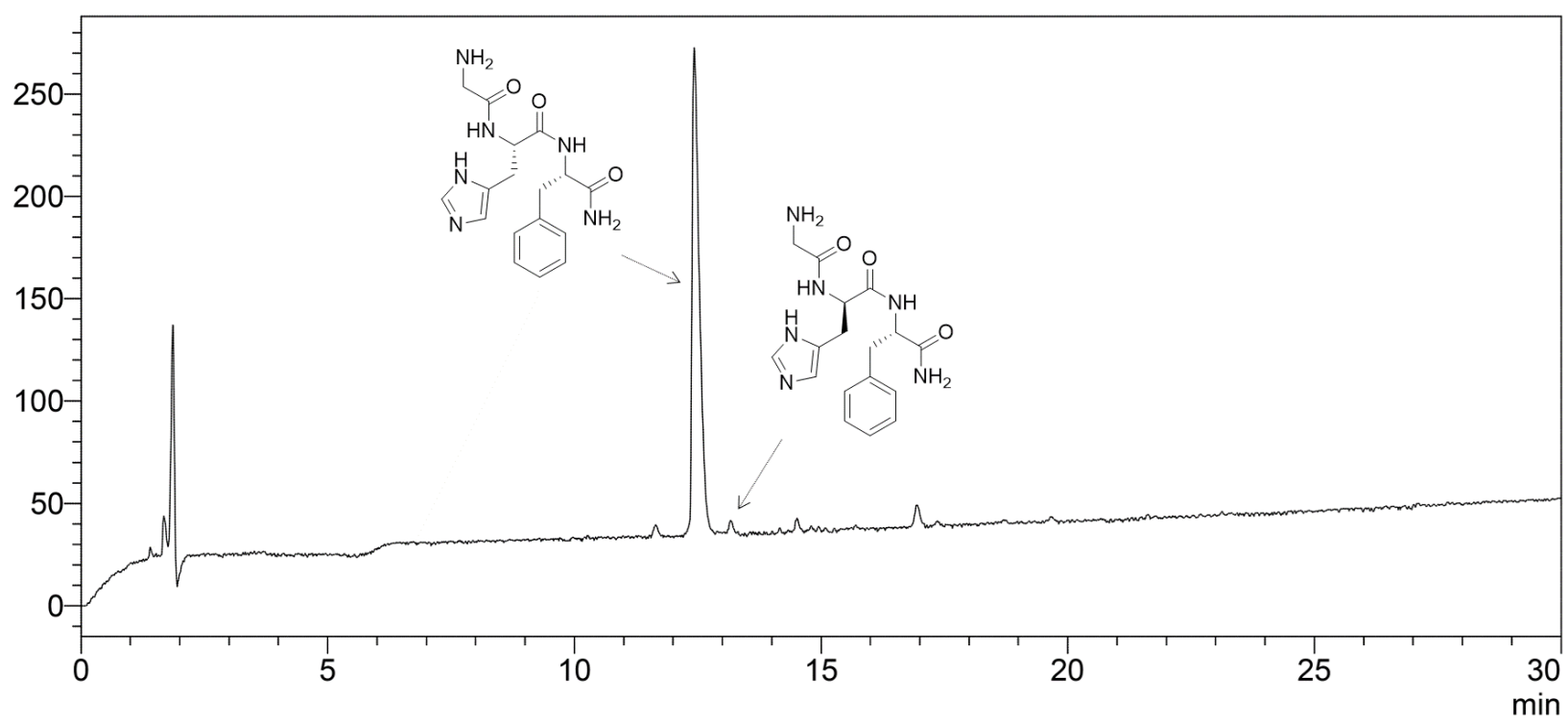

Figure S32. Chromatogram of entry 4 (Table 2) obtained by RP-HPLC (Shimadzu Prominance UFLC, SPD-M20A/DGU-20A3R/LC-20AD) on a Phenomenex Kinetex column (C18, $150 \mathrm{~mm} \times 4.6 \mathrm{~mm}, 5 \mu \mathrm{m}$, $100 \AA$ A). Purity check/Gradient $2-\mathrm{t}_{\mathrm{R}}=12.4 \mathrm{~min}$.

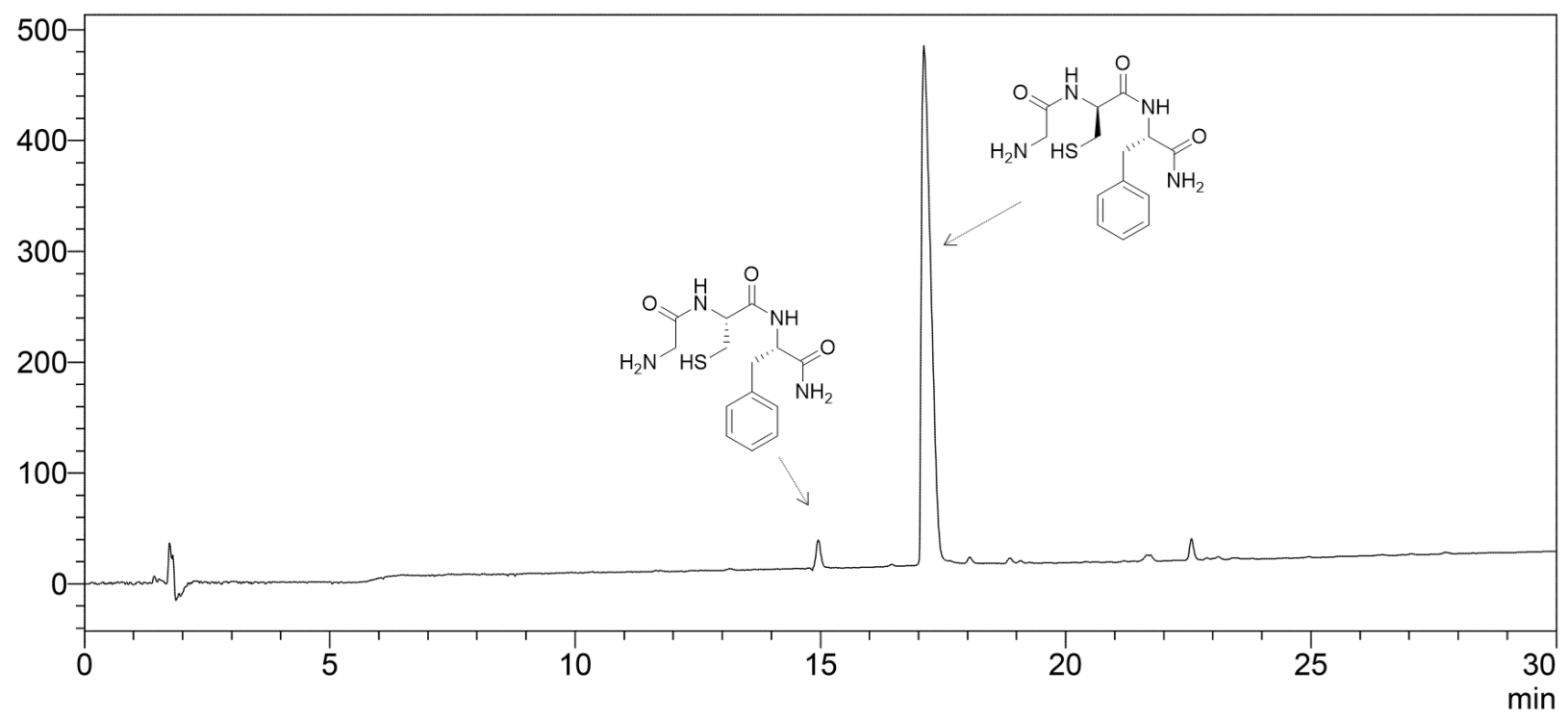

Figure S33. Chromatogram of entry 5 (Table 2) obtained by RP-HPLC (Shimadzu Prominance UFLC, SPD-M20A/DGU-20A3R/LC-20AD) on a Phenomenex Kinetex column $(\mathrm{C} 18,150 \mathrm{~mm} \times 4.6 \mathrm{~mm}, 5 \mu \mathrm{m}$, $100 \AA$ ). Purity check/Gradient $2-\mathrm{t}_{\mathrm{R}}=17.1 \mathrm{~min}$. 


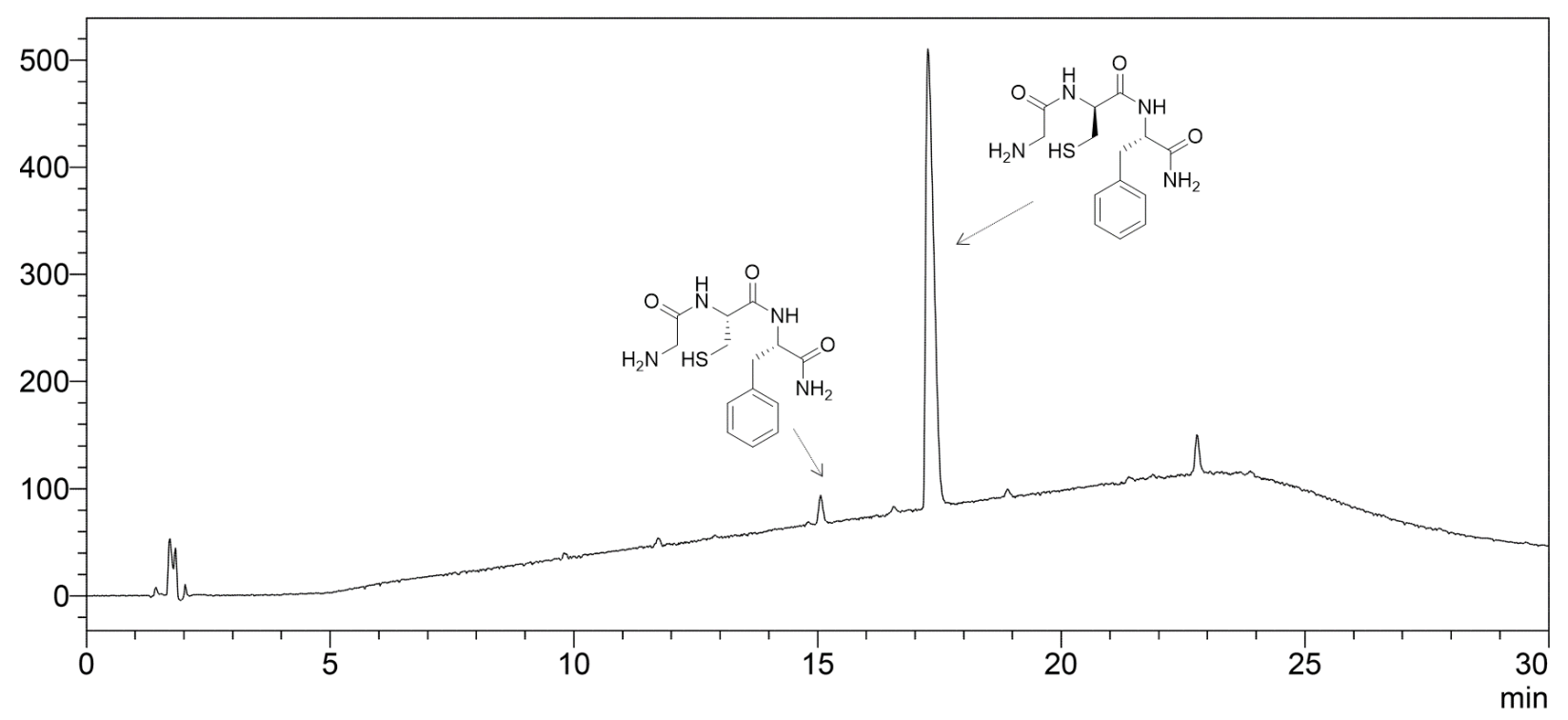

Figure S34. Chromatogram of entry 6 (Table 2) obtained by RP-HPLC (Shimadzu Prominance UFLC, SPD-M20A/DGU-20A3R/LC-20AD) on a Phenomenex Kinetex column (C18, $150 \mathrm{~mm} \times 4.6 \mathrm{~mm}, 5 \mu \mathrm{m}$, $100 \AA$ ). Purity check/Gradient $2-\mathrm{t}_{\mathrm{R}}=17.1 \mathrm{~min}$.

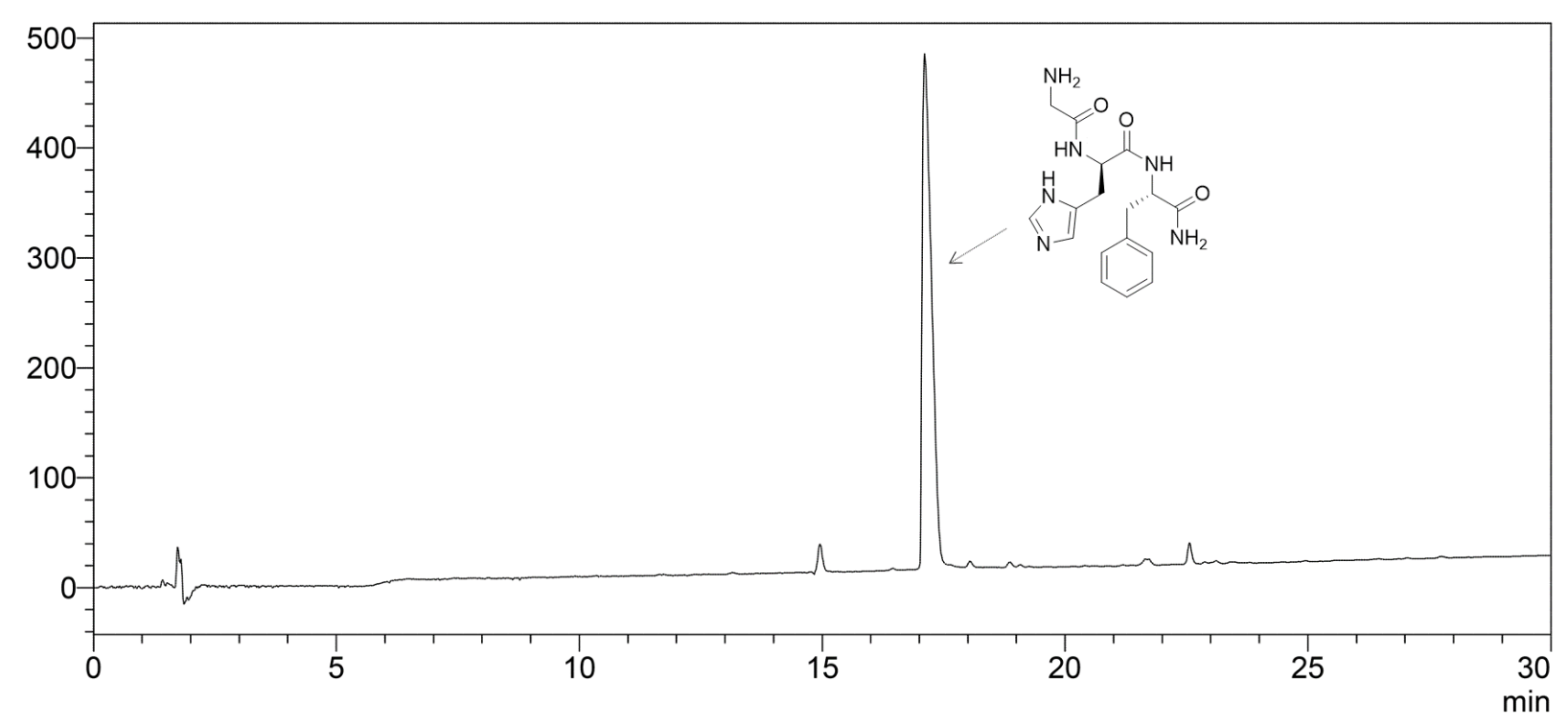

Figure S35. Chromatogram of entry 7 (Table 2) obtained by RP-HPLC (Shimadzu Prominance UFLC, SPD-M20A/DGU-20A3R/LC-20AD) on a Phenomenex Kinetex column (C18, $150 \mathrm{~mm} \times 4.6 \mathrm{~mm}, 5 \mu \mathrm{m}$, $100 \AA$ ). Purity check/Gradient $2-\mathrm{t}_{\mathrm{R}}=12.8 \mathrm{~min}$. 


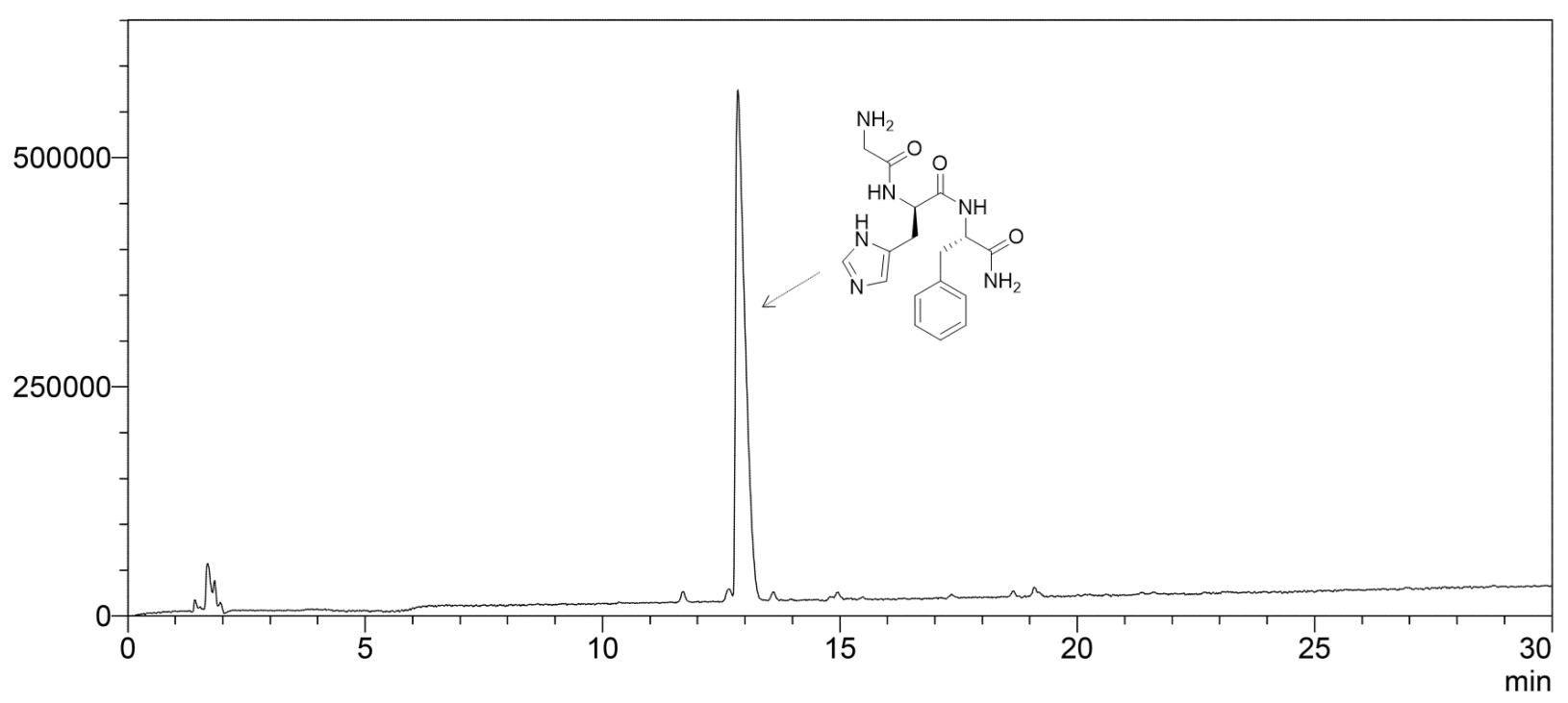

Figure S36. Chromatogram of entry 8 (Table 2) obtained by RP-HPLC (Shimadzu Prominance UFLC, SPD-M20A/DGU-20A3R/LC-20AD) on a Phenomenex Kinetex column (C18, $150 \mathrm{~mm} \times 4.6 \mathrm{~mm}, 5 \mu \mathrm{m}$, $100 \AA$ ). Purity check/Gradient $2-t_{R}=12.8$ min.

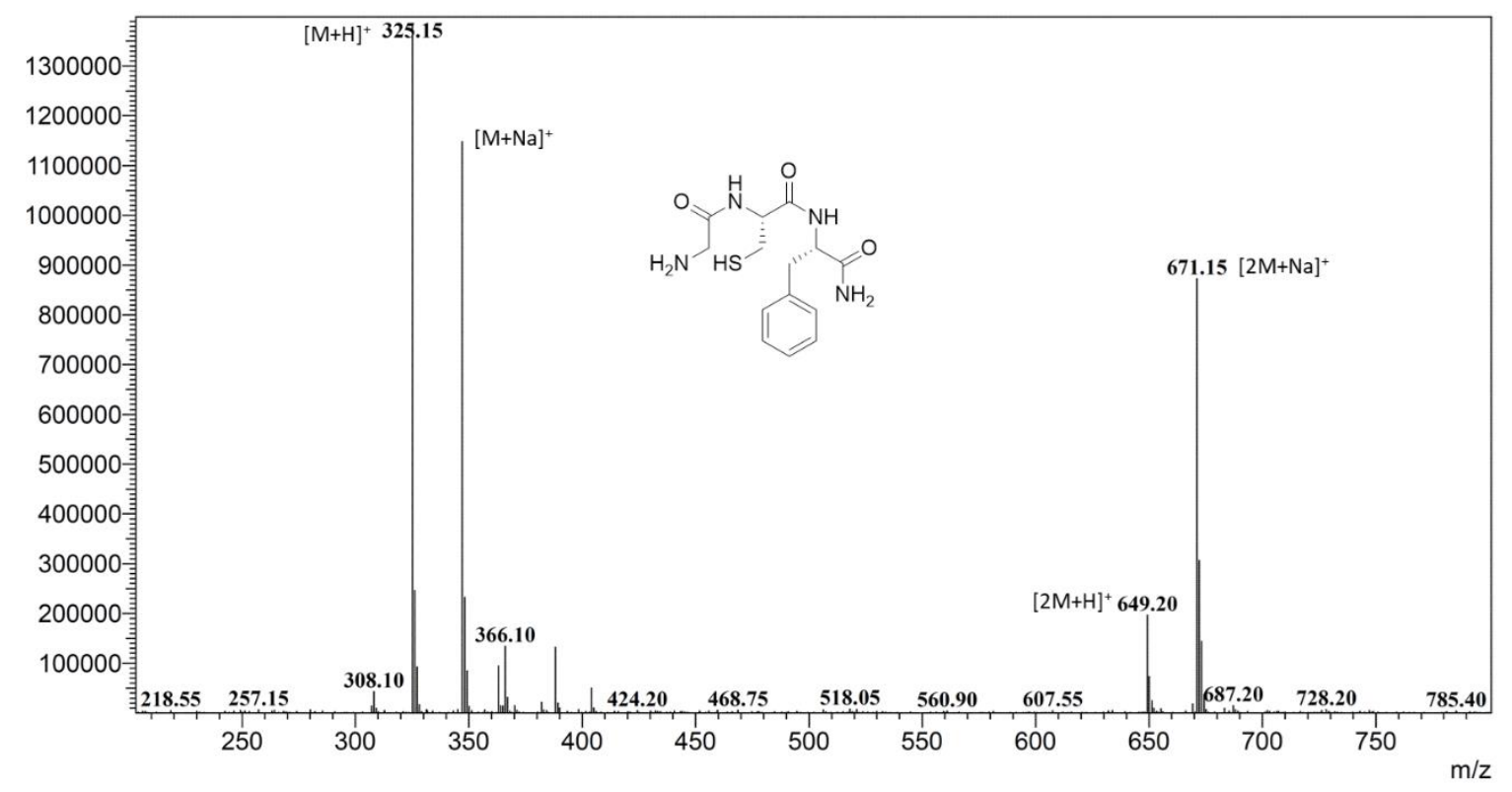

Figure S37. ESI-MS spectrum of peak at $t_{R}=14.9$ min resulted from chromatograms of entries 1 and 2 (Table 2). 


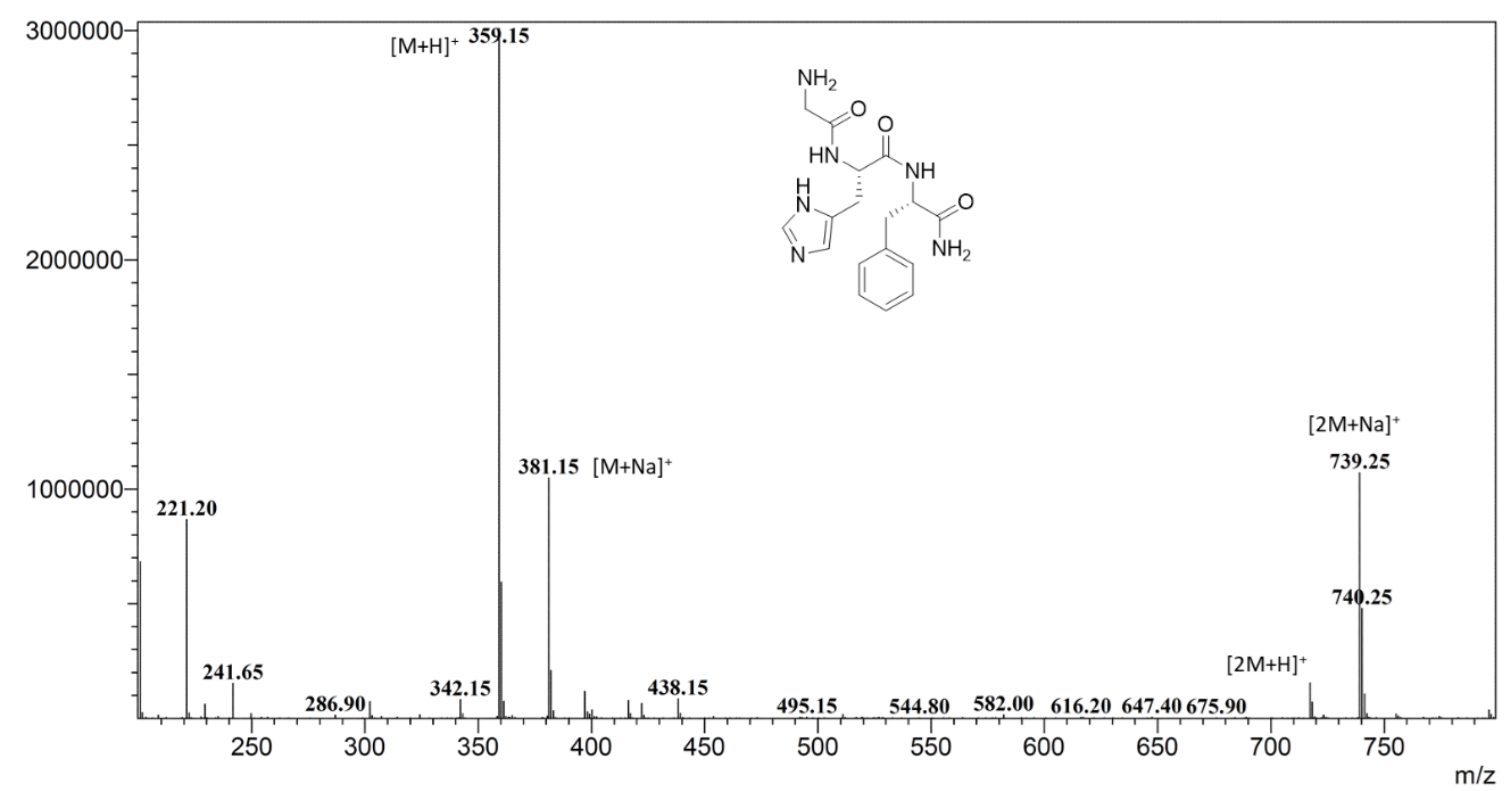

Figure S38. ESI-MS spectrum of peak at $t_{R}=12.4$ min resulted from chromatograms of entries 3 and 4 (Table 2).

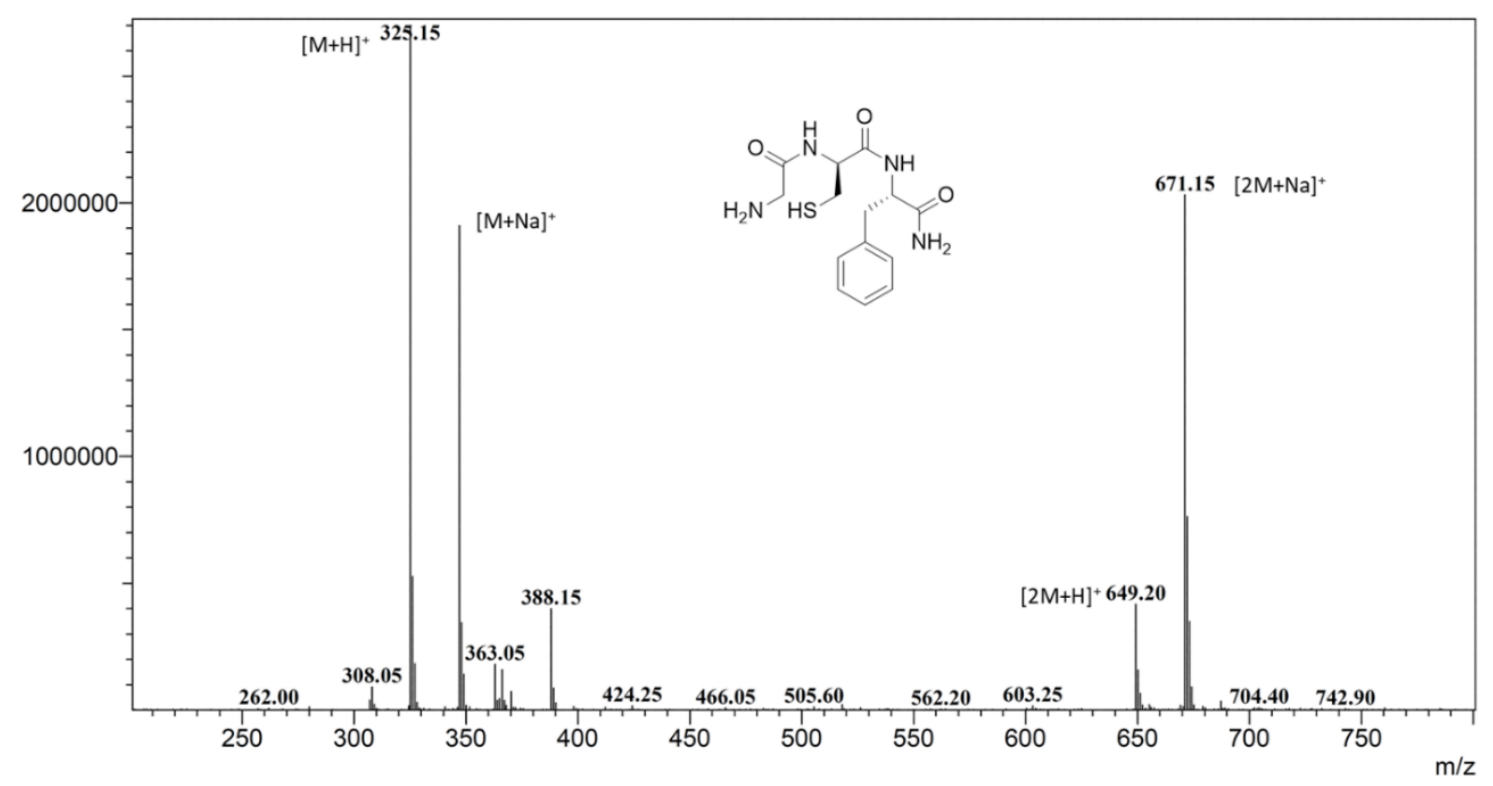

Figure S39. ESI-MS spectrum of peak at $t_{R}=17.1$ min resulted from chromatograms of entries 5 and $\mathbf{6}$ (Table 2). 


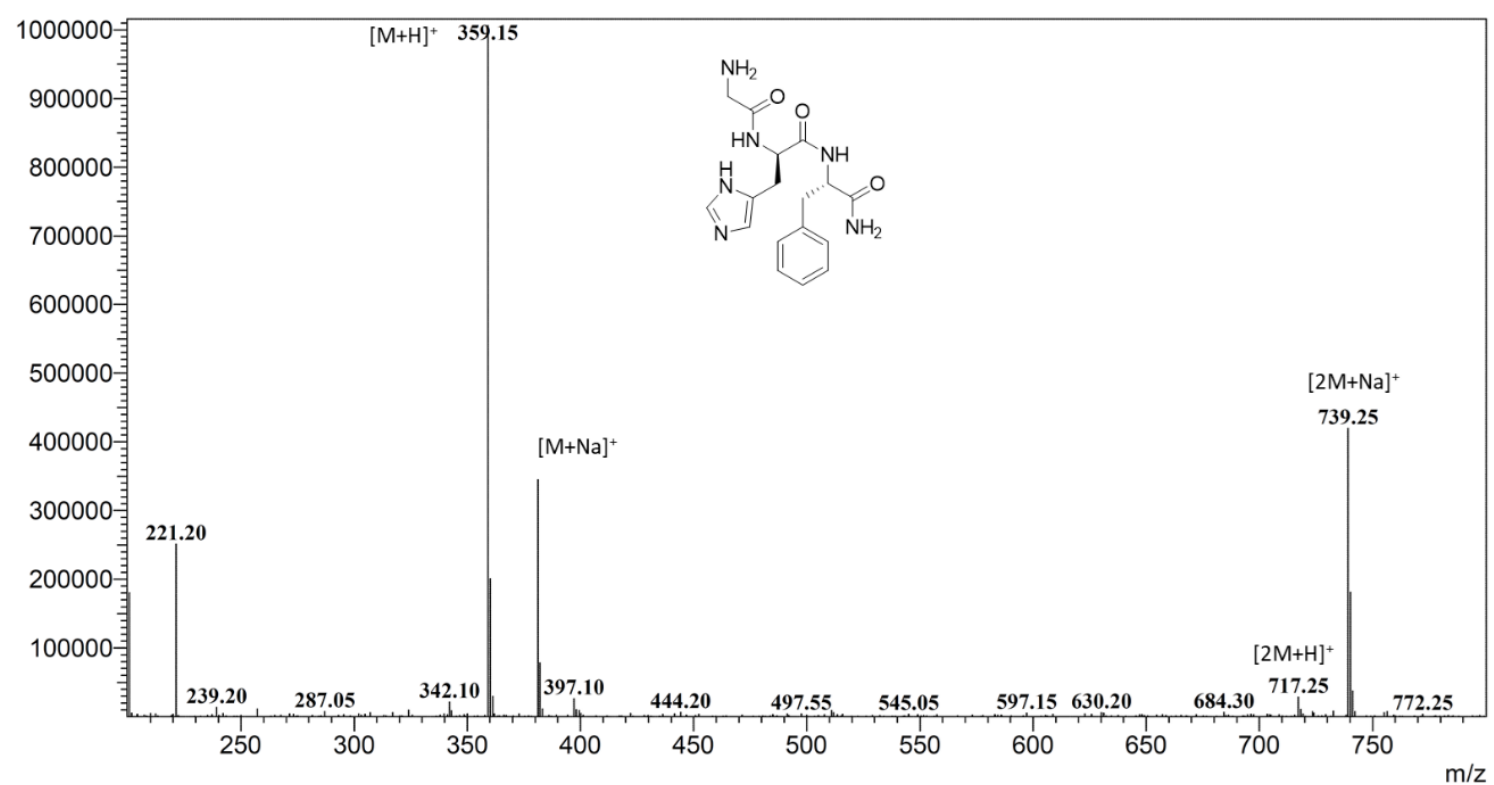

Figure S40. ESI-MS spectrum of peak at $t_{R}=12.8$ min resulted from chromatograms of entries $\mathbf{7}$ and $\mathbf{8}$ (Table 2).

Biologically relevant peptides (Table 3, entries 1-9)

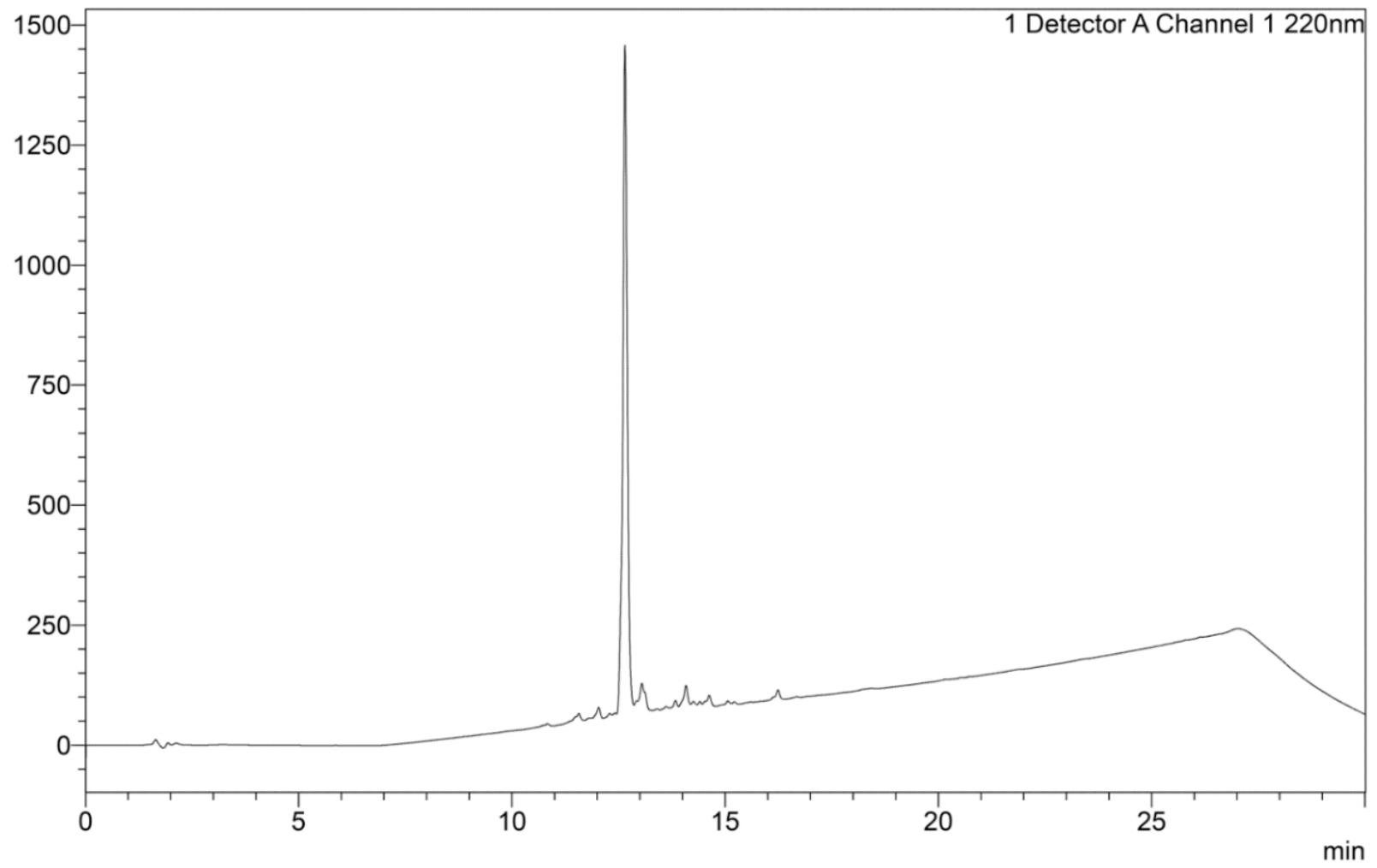

Figure S41. Chromatogram of entry 1 (Kisspeptin-10, Table 3) obtained by RP-HPLC (Shimadzu Prominance UFLC, SPD-M20A/DGU-20A3R/LC-20AD) on a Phenomenex Kinetex column (C18, 150 $\mathrm{mm} \times 4.6 \mathrm{~mm}, 5 \mu \mathrm{m}, 100 \AA$ ). Purity check/Gradient $1-\mathrm{t}_{\mathrm{R}}=12.5 \mathrm{~min}$. 


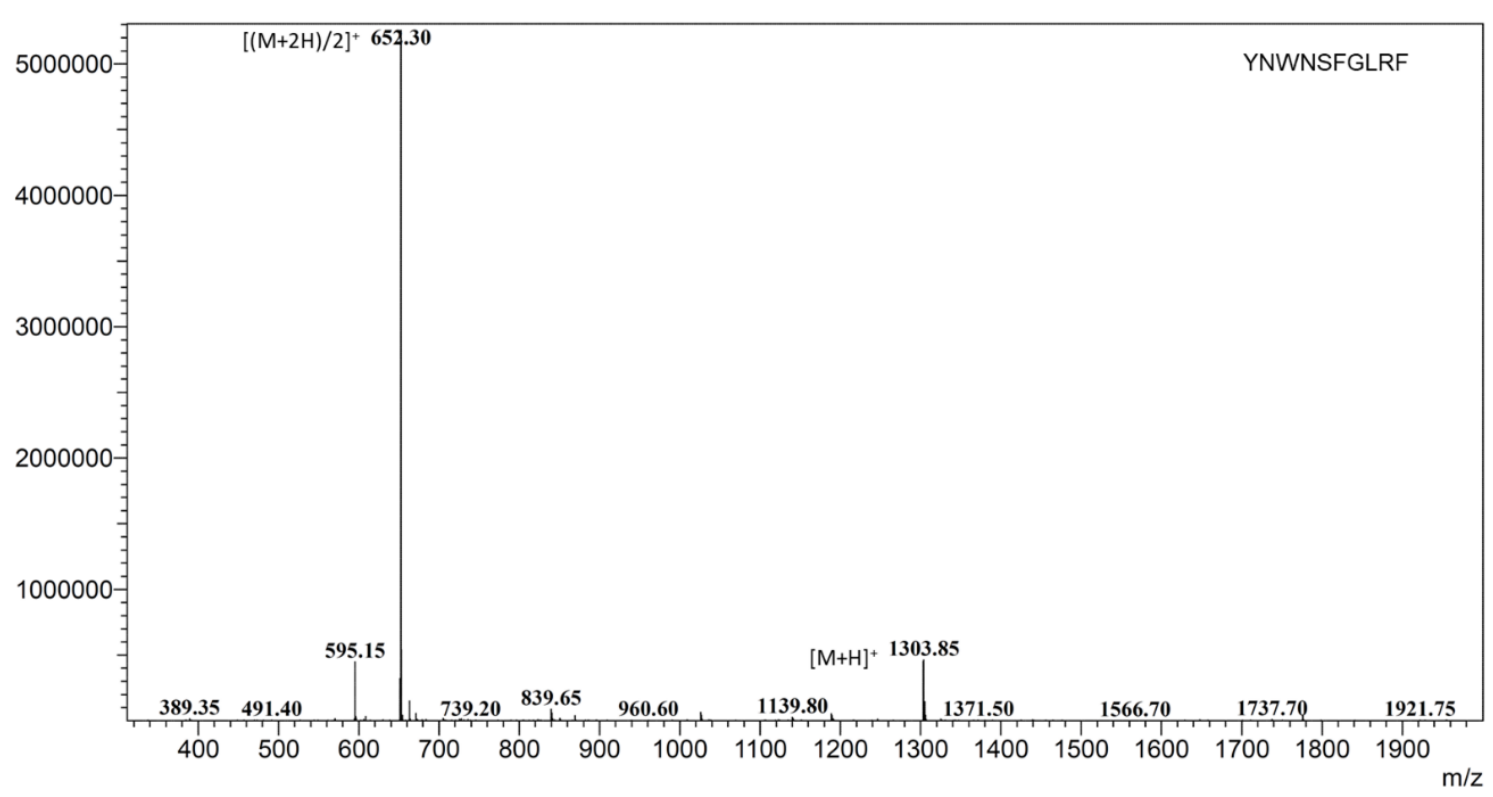

Figure S42. ESI-MS spectrum of peak at $t_{R}=12.5$ min resulted from chromatogram of entry 1 (Kisspeptin-10, Table 3).

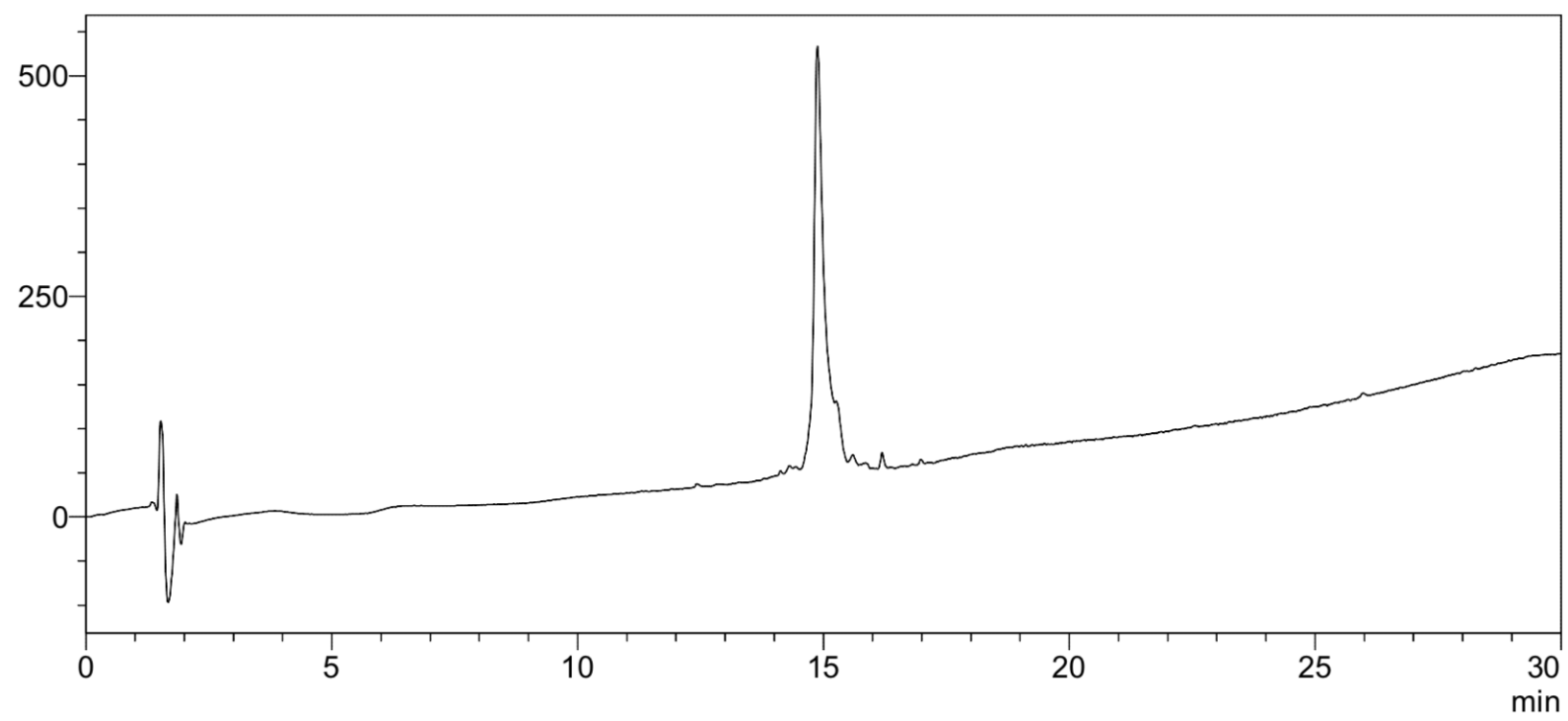

Figure S43. Chromatogram of entry 2 (Angiotensin-I, Table 3) obtained by RP-HPLC (Shimadzu Prominance UFLC, SPD-M20A/DGU-20A3R/LC-20AD) on a Phenomenex Kinetex column (C18, 150 $\mathrm{mm} \times 4.6 \mathrm{~mm}, 5 \mu \mathrm{m}, 100 \AA$ ). Purity check/Gradient $1-\mathrm{t}_{\mathrm{R}}=14.9 \mathrm{~min}$. 


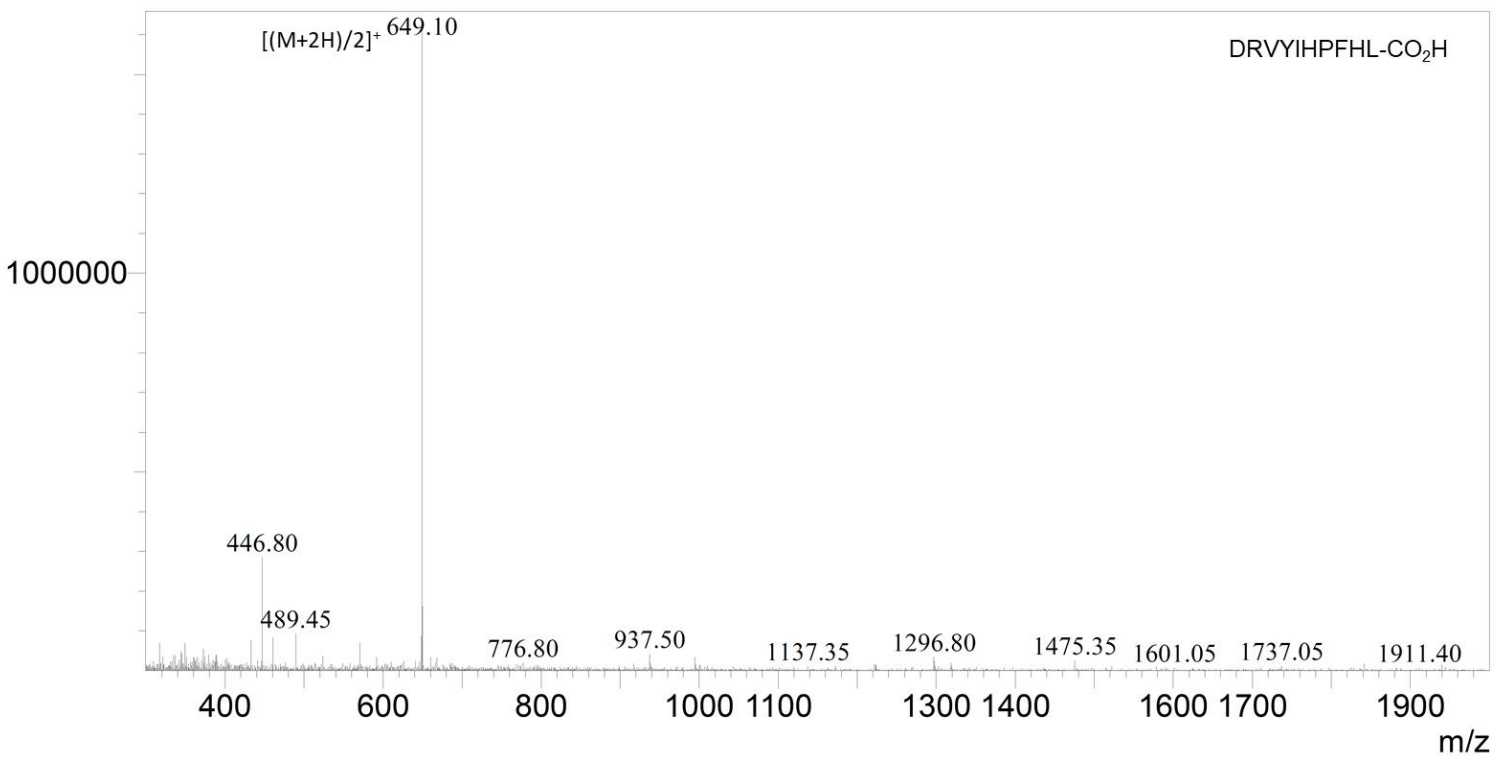

Figure S44. ESI-MS spectrum of peak at $t_{R}=14.9$ min resulted from chromatogram of entry 2 (Angiotensin-I, Table 3).

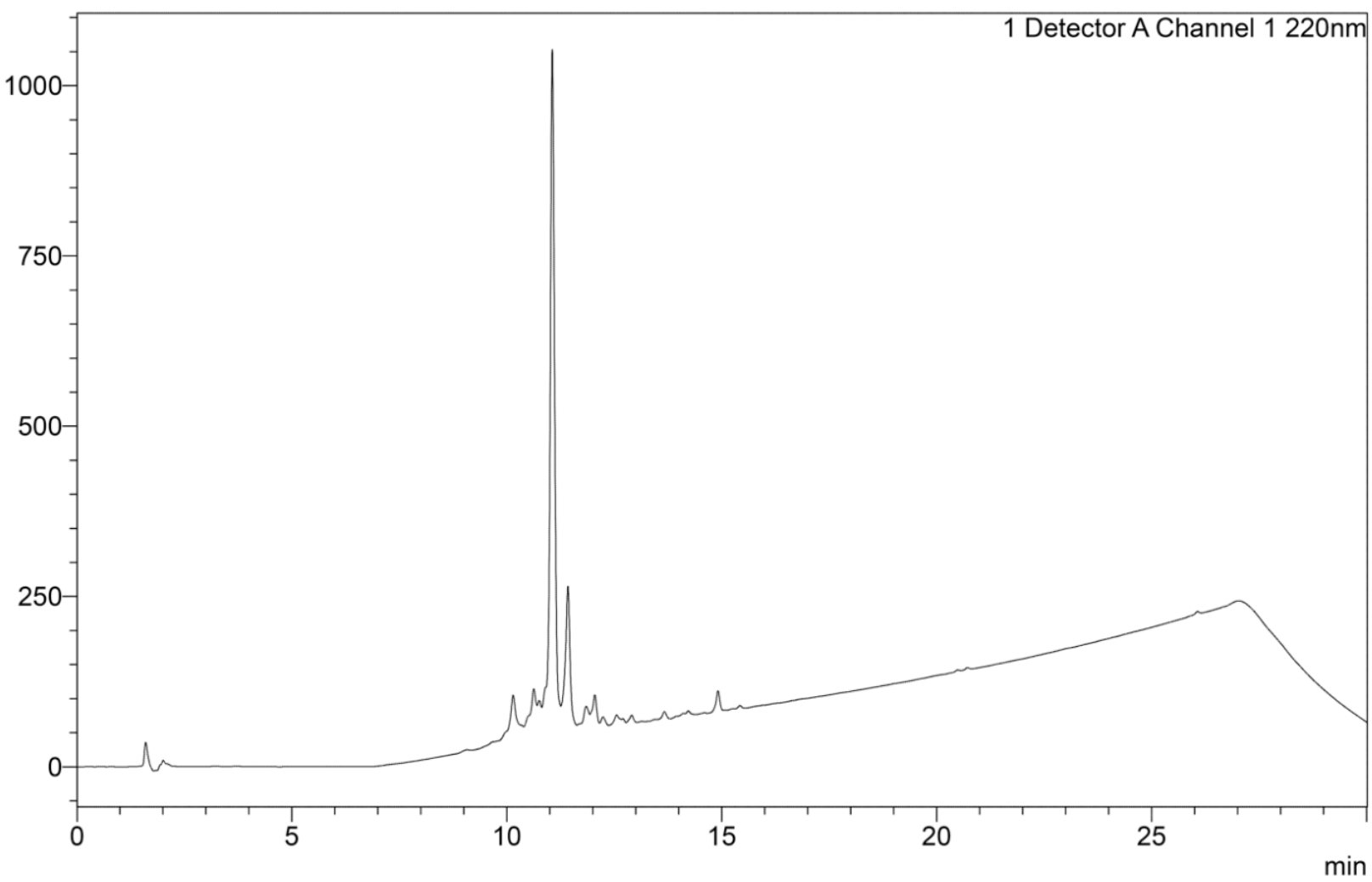

Figure S45. Chromatogram of entry 3 ( $\alpha$-MSH, Table 3 ) obtained by RP-HPLC (Shimadzu Prominance UFLC, SPD-M20A/DGU-20A3R/LC-20AD) on a Phenomenex Kinetex column $(\mathrm{C} 18,150 \mathrm{~mm} \times 4.6$ $\mathrm{mm}, 5 \mu \mathrm{m}, 100 \AA ̊)$. Purity check/Gradient $1-\mathrm{t}_{\mathrm{R}}=11.0 \mathrm{~min}$. 


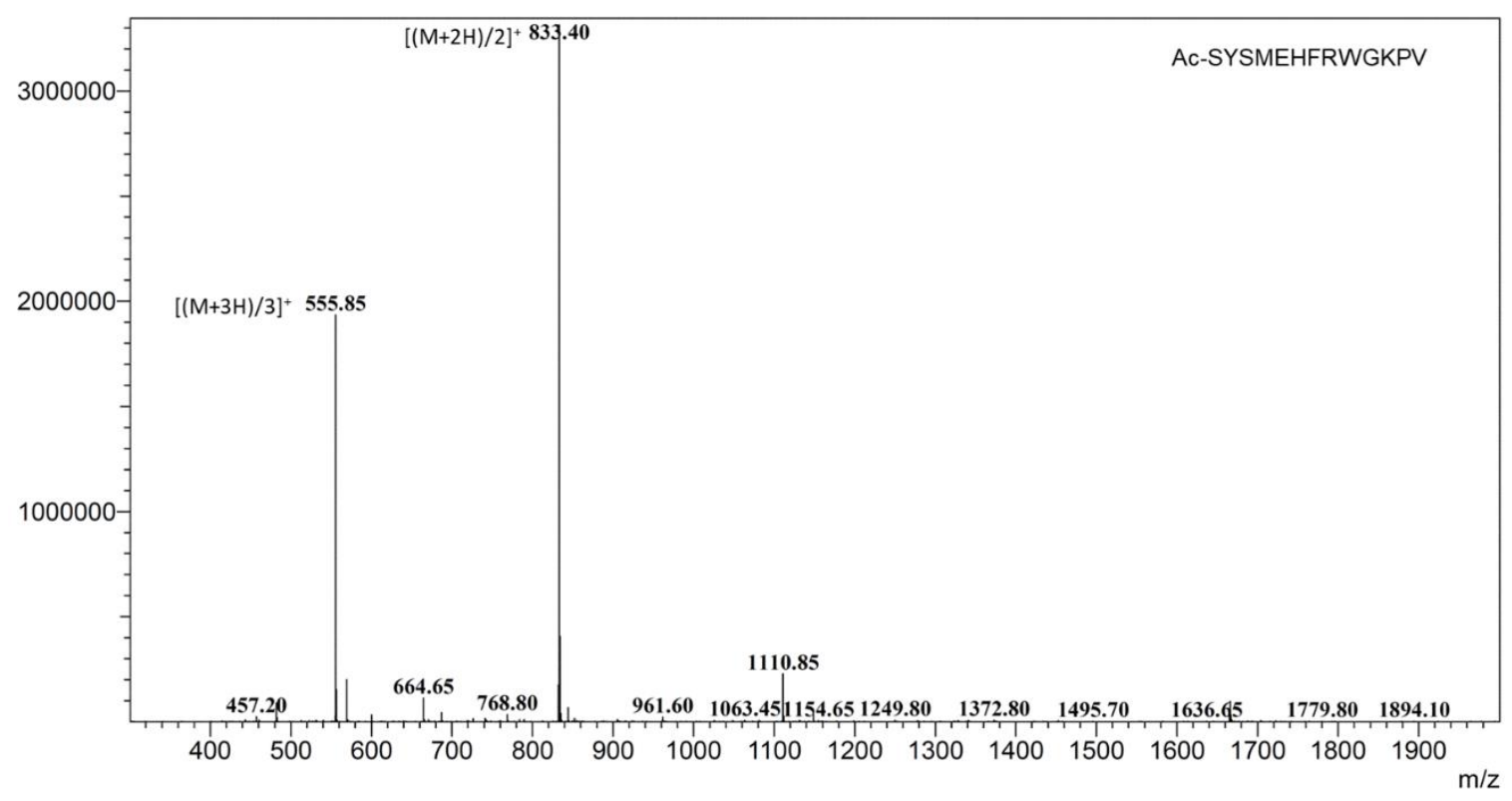

Figure S46. ESI-MS spectrum of peak at $t_{R}=11.0$ min resulted from chromatogram of entry $3(\alpha-M S H$, Table 3).

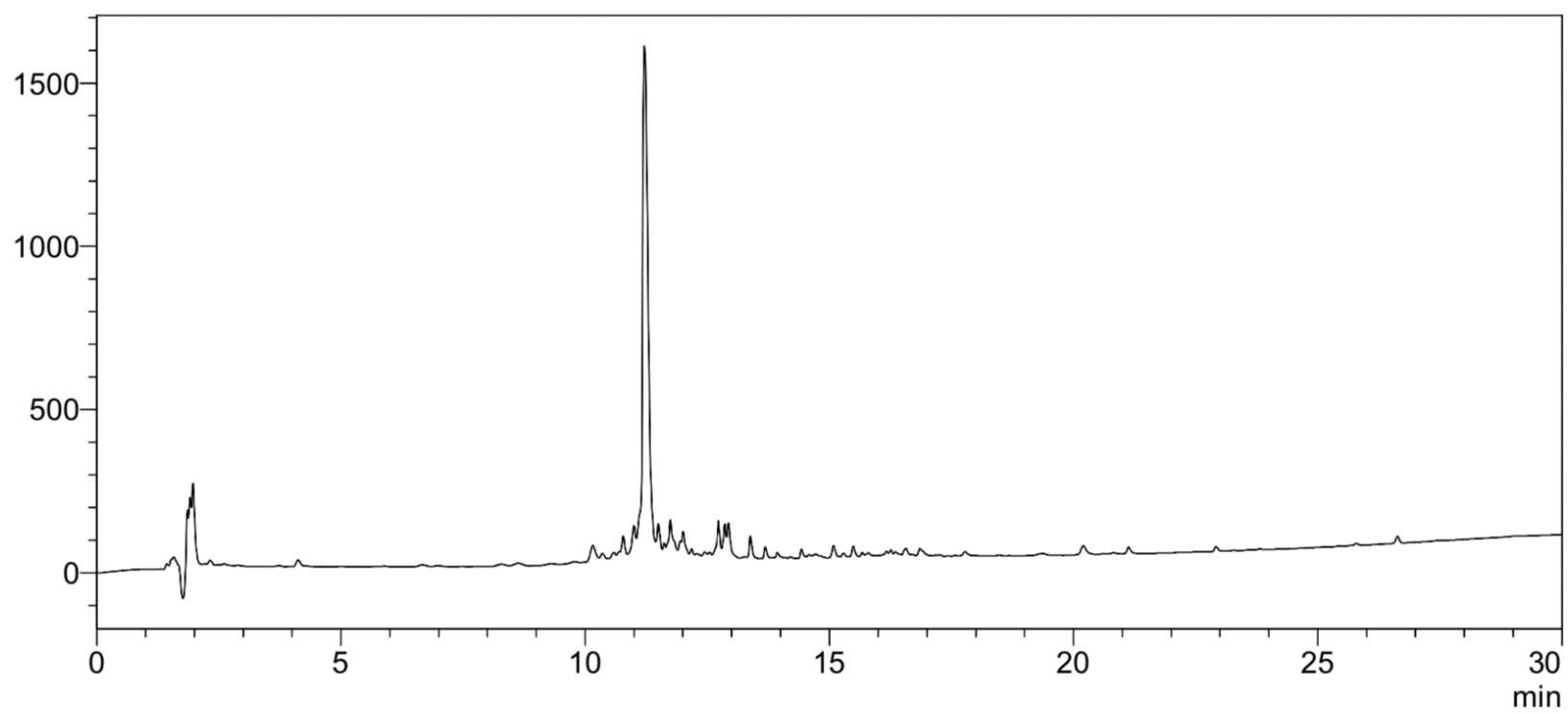

Figure S47. Chromatogram of entry 4 (PEPITEM, Table 3) obtained by RP-HPLC (Shimadzu Prominance UFLC, SPD-M20A/DGU-20A3R/LC-20AD) on a Phenomenex Kinetex column (C18, 150 $\mathrm{mm} \times 4.6 \mathrm{~mm}, 5 \mu \mathrm{m}, 100 \AA$ ). Purity check/Gradient $1-\mathrm{t}_{\mathrm{R}}=11.2 \mathrm{~min}$. 


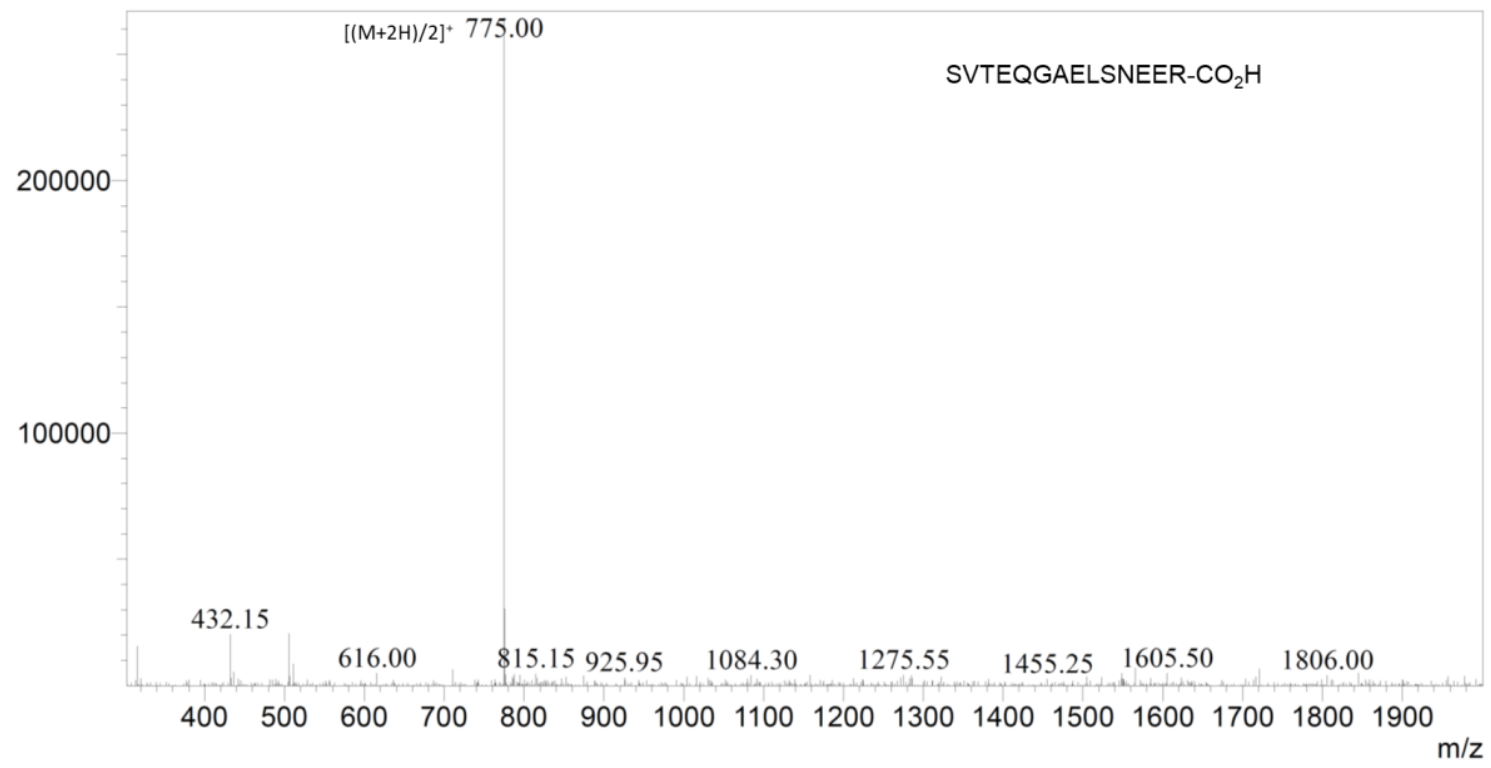

Figure S48. ESI-MS spectrum of peak at $\mathrm{tR}=11.2 \mathrm{~min}$ resulted from chromatogram of entry 4 (PEPITEM, Table 3).

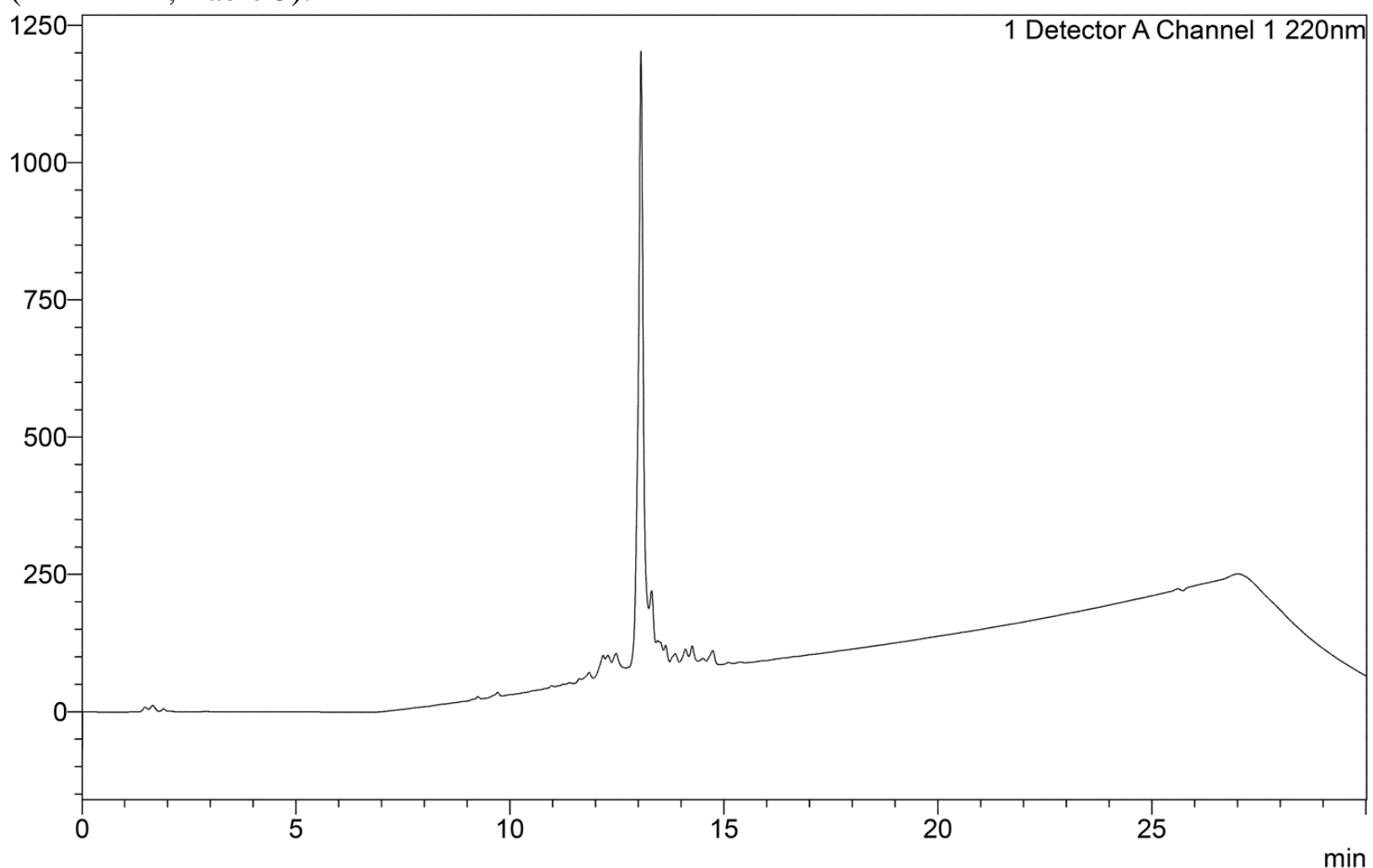

Figure S49. Chromatogram of entry 5 (p53-TAD $15-29$, Table 3 ) obtained by RP-HPLC (Shimadzu Prominance UFLC, SPD-M20A/DGU-20A3R/LC-20AD) on a Phenomenex Kinetex column (C18, 150 $\mathrm{mm} \times 4.6 \mathrm{~mm}, 5 \mu \mathrm{m}, 100 \AA$ ). Purity check/Gradient $1-\mathrm{t}_{\mathrm{R}}=13.1 \mathrm{~min}$. 


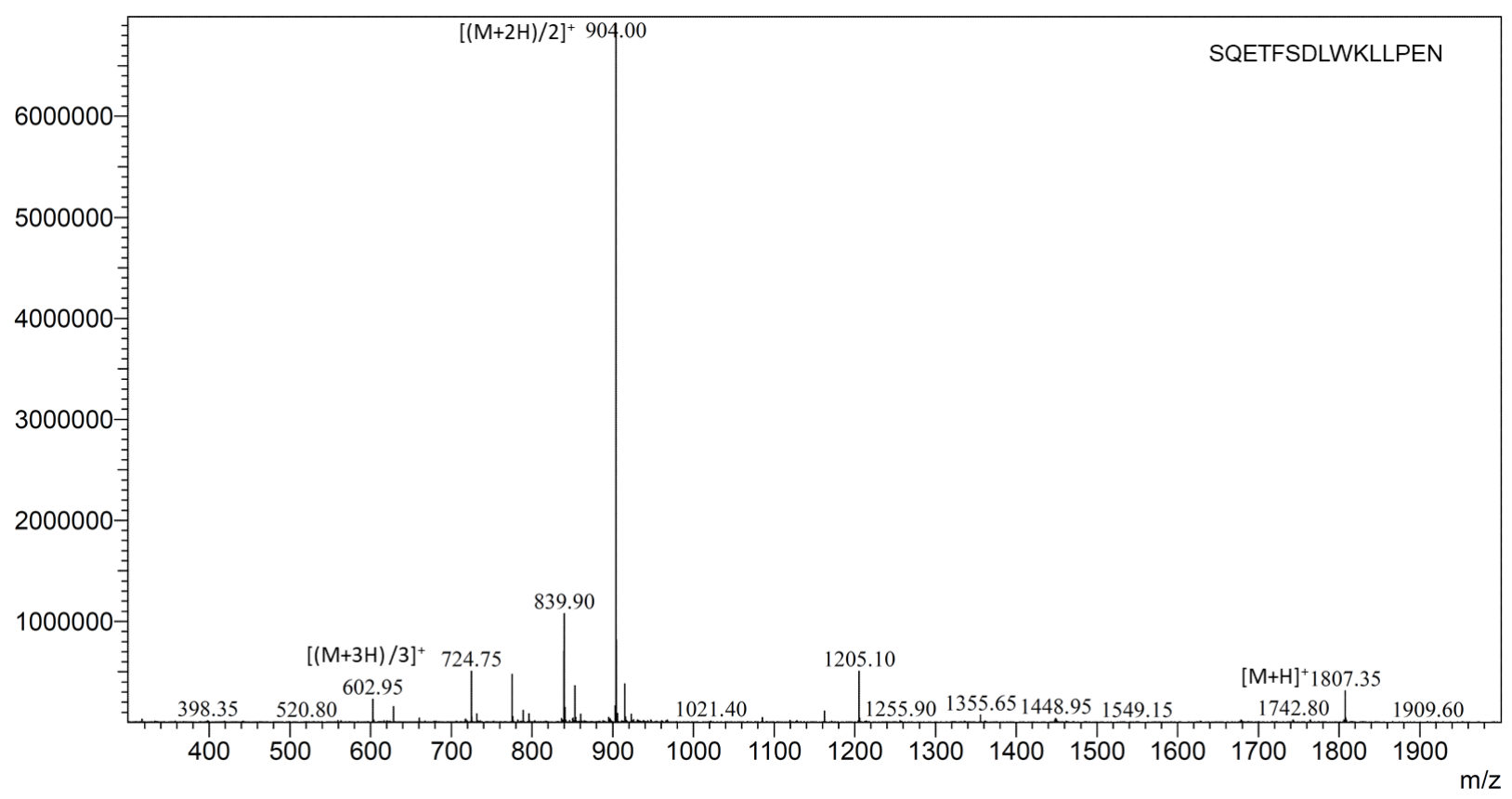

Figure S50. ESI-MS spectrum of peak at $t_{R}=13.1$ min resulted from chromatogram of entry 5 (p53TAD $15-29$, Table 3).

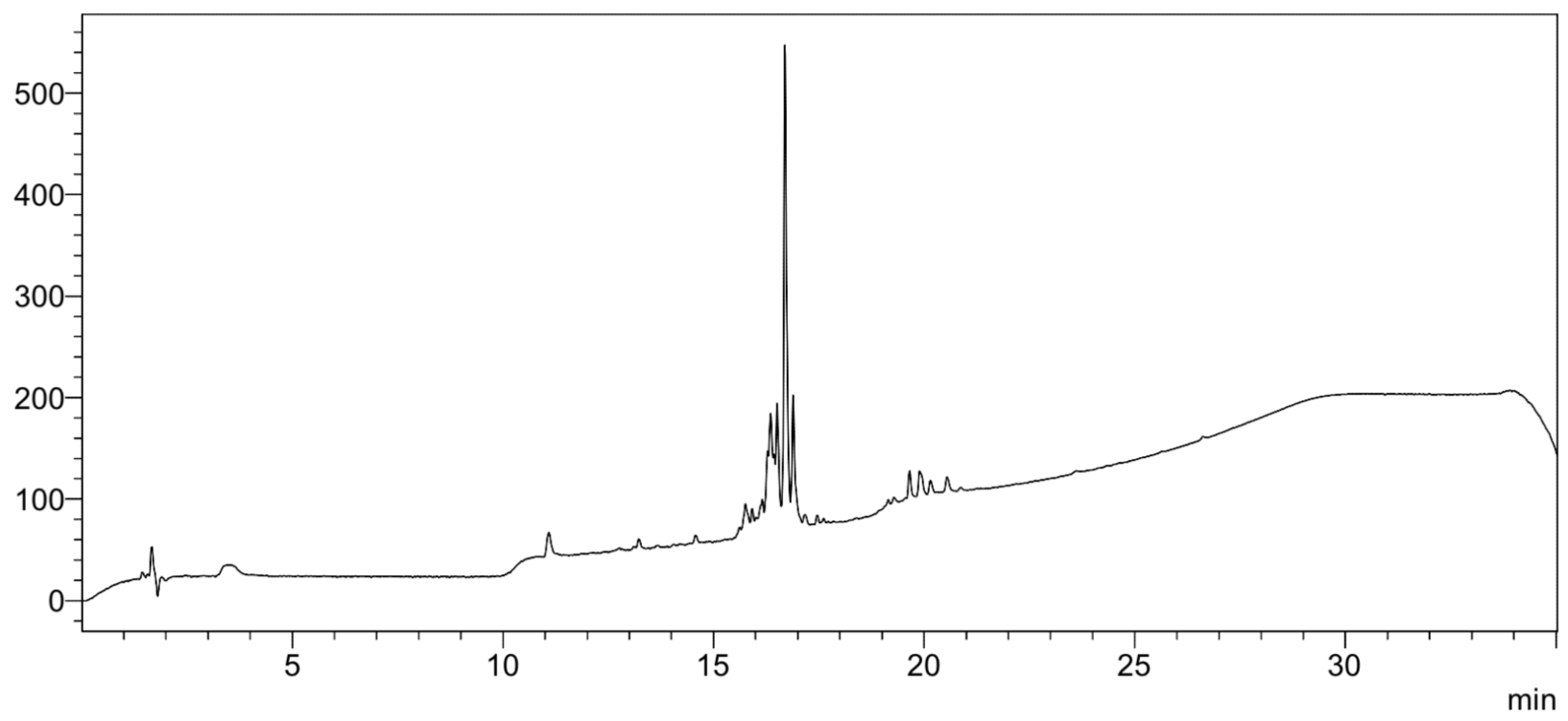

Figure S51. Chromatogram of entry 6 ( $\gamma$-endorphin, Table 3) obtained by RP-HPLC (Shimadzu Prominance UFLC, SPD-M20A/DGU-20A3R/LC-20AD) on a Phenomenex Kinetex column (C18, 150 $\mathrm{mm} \times 4.6 \mathrm{~mm}, 5 \mu \mathrm{m}, 100 \AA$ ). Purity check/Gradient $1-\mathrm{t}_{\mathrm{R}}=16.7 \mathrm{~min}$. 


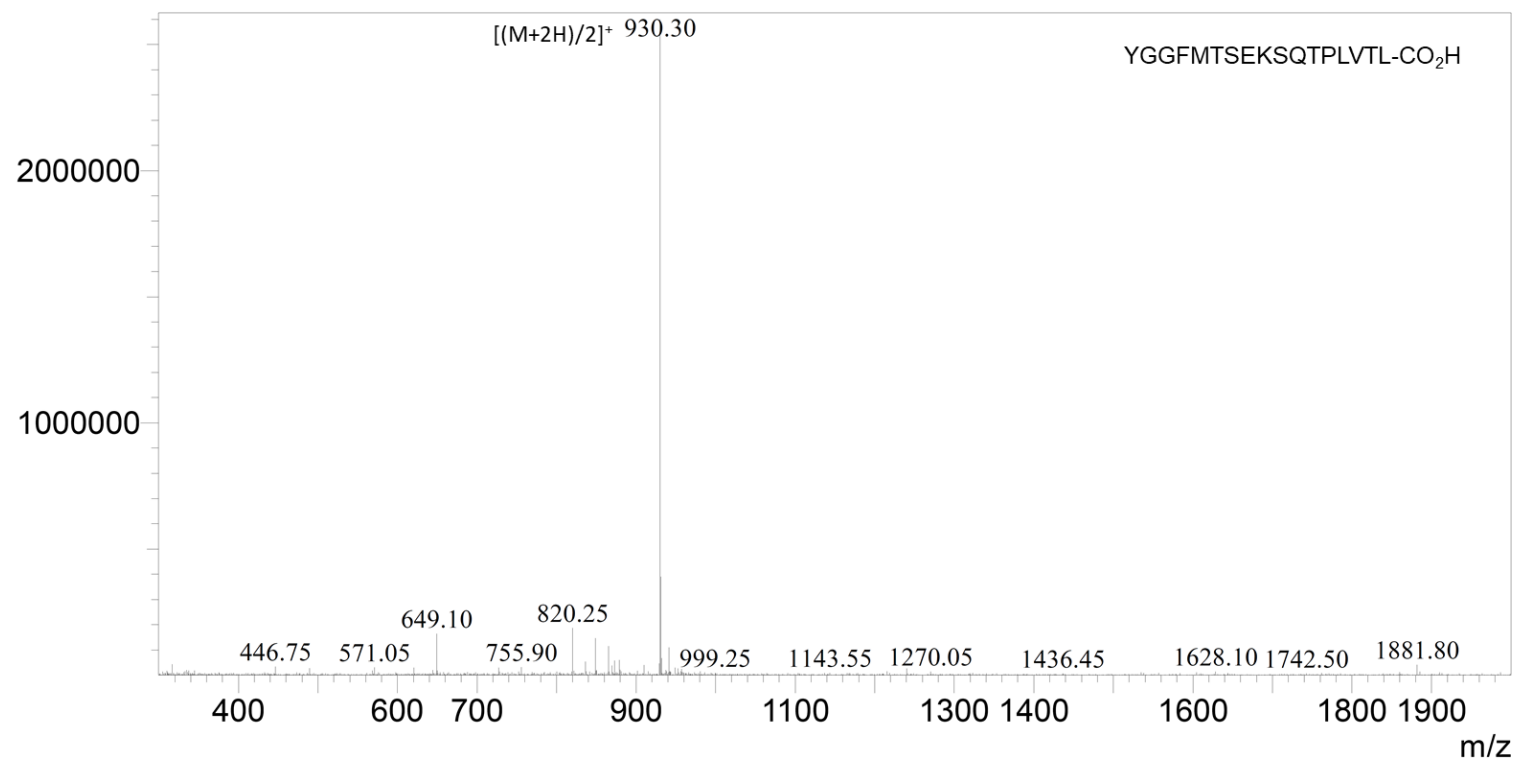

Figure S52. ESI-MS spectrum of peak at $t_{R}=16.7$ min resulted from chromatogram of entry $6(\gamma$ endorphin, Table 3).

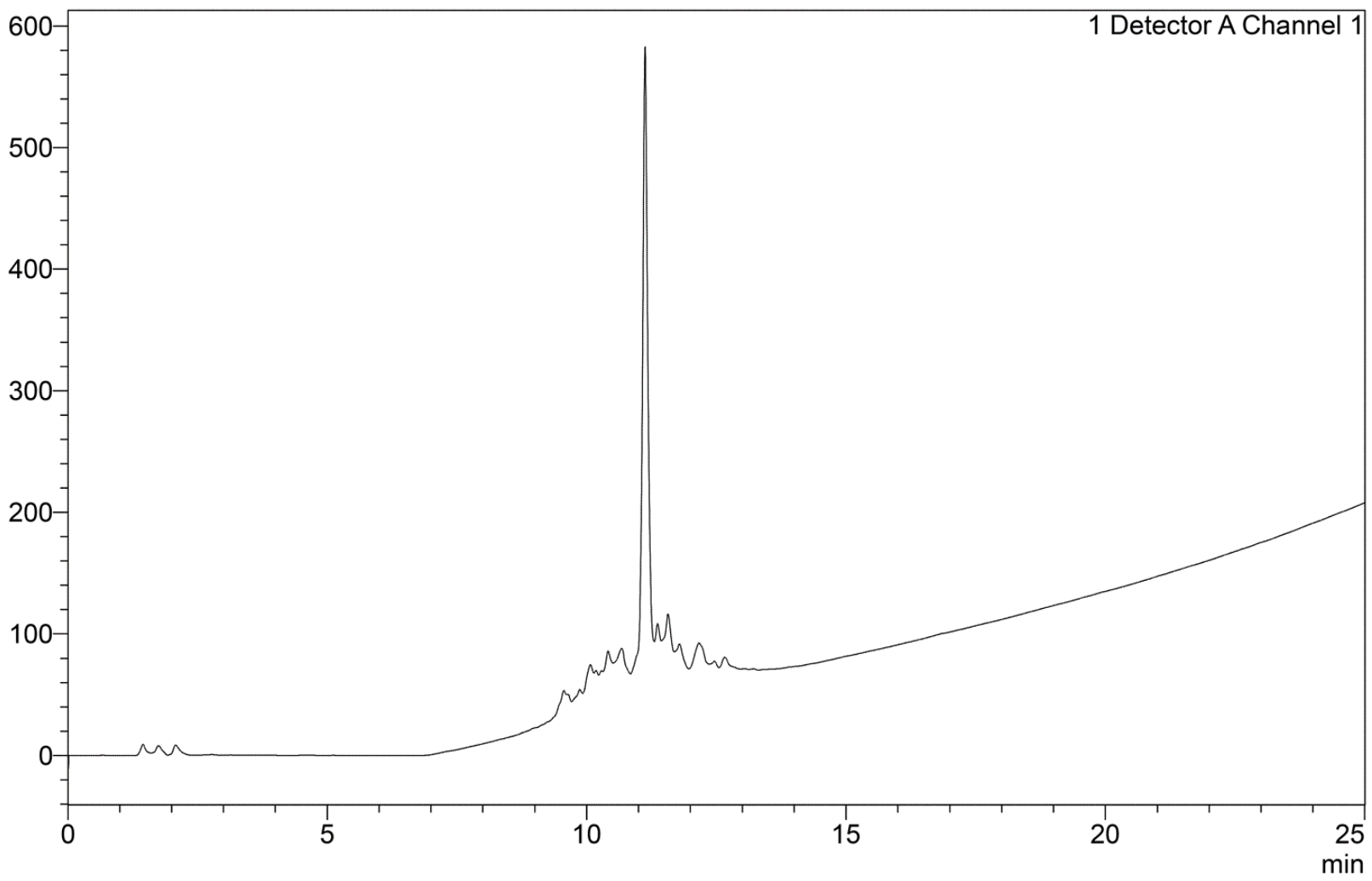

Figure S53. Chromatogram of entry 7 (PAMP ${ }_{1-20}$, Table 3) obtained by RP-HPLC (Shimadzu Prominance UFLC, SPD-M20A/DGU-20A3R/LC-20AD) on a Phenomenex Kinetex column (C18, 150 $\mathrm{mm} \times 4.6 \mathrm{~mm}, 5 \mu \mathrm{m}, 100 \AA$ ). Purity check/Gradient $1-\mathrm{t}_{\mathrm{R}}=11.1 \mathrm{~min}$. 


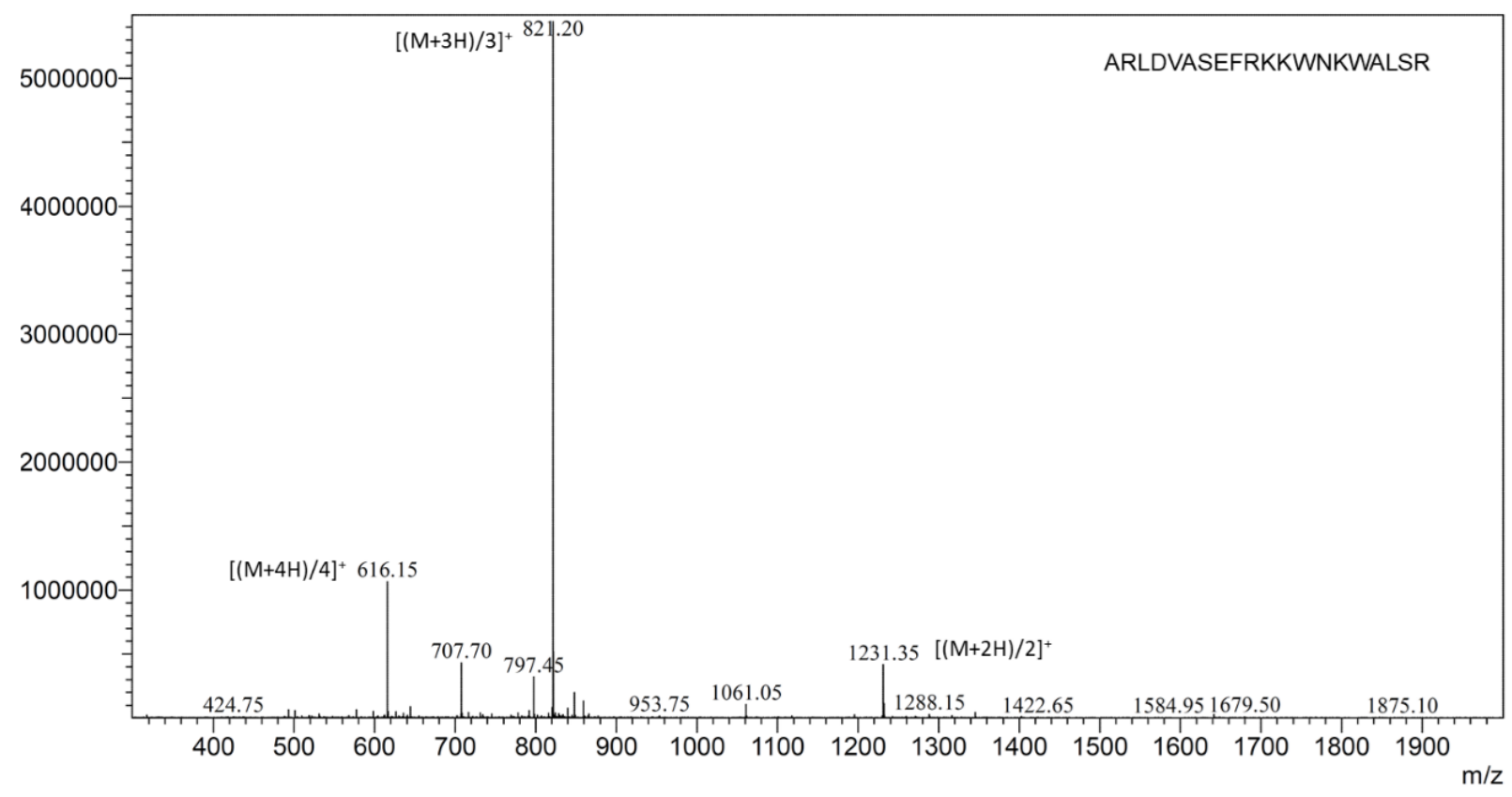

Figure S54. ESI-MS spectrum of peak at $t_{R}=11.1$ min resulted from chromatogram of entry $7\left(\mathrm{PAMP}_{1}\right.$ 20, Table 3).

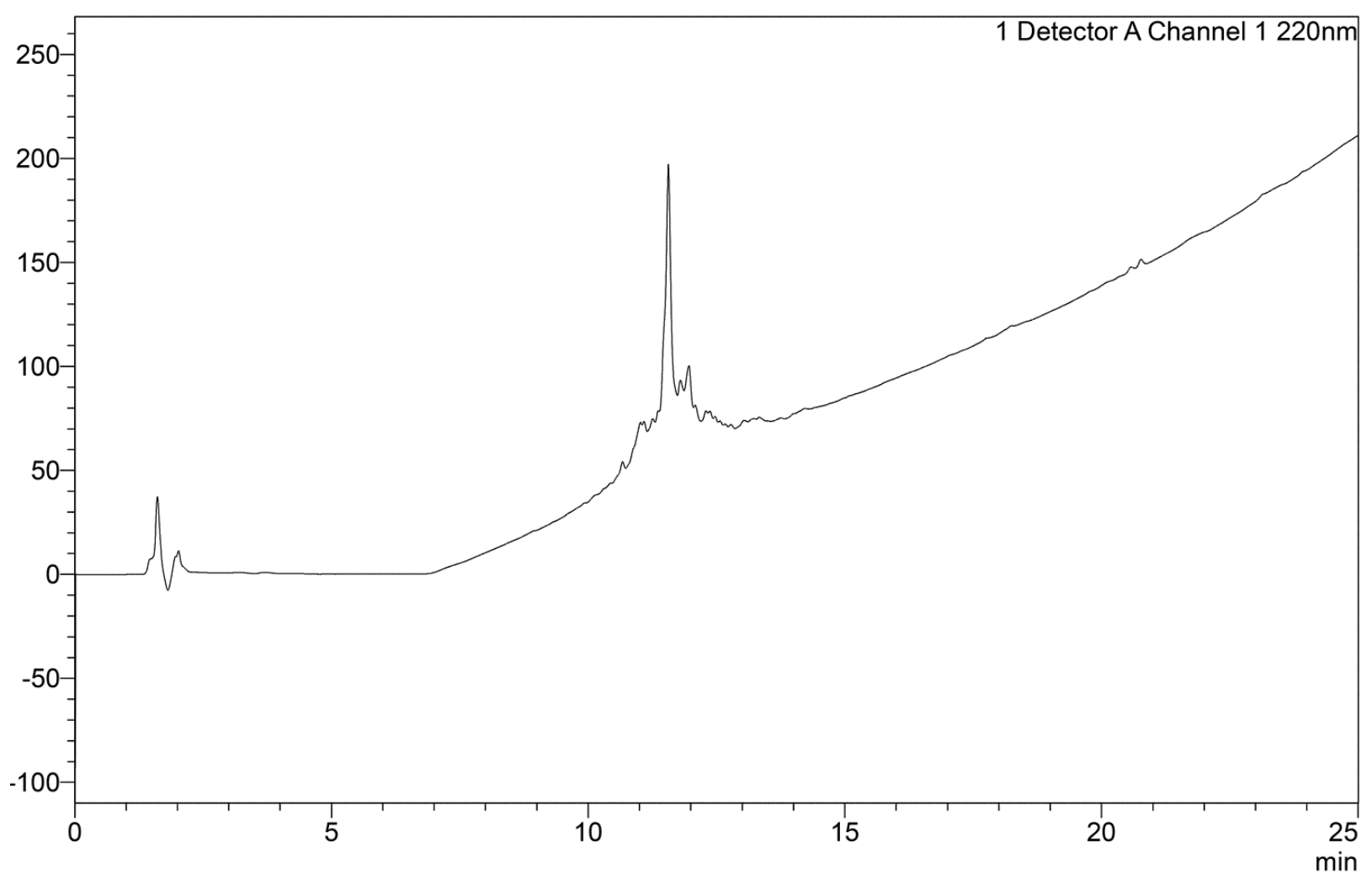

Figure S55. Chromatogram of entry 8 (VIP, Table 3) obtained by RP-HPLC (Shimadzu Prominance UFLC, SPD-M20A/DGU-20A3R/LC-20AD) on a Phenomenex Kinetex column $(\mathrm{C} 18,150 \mathrm{~mm} \times 4.6$ $\mathrm{mm}, 5 \mu \mathrm{m}, 100 \AA$ ). Purity check/Gradient $1-\mathrm{t}_{\mathrm{R}}=11.5 \mathrm{~min}$. 


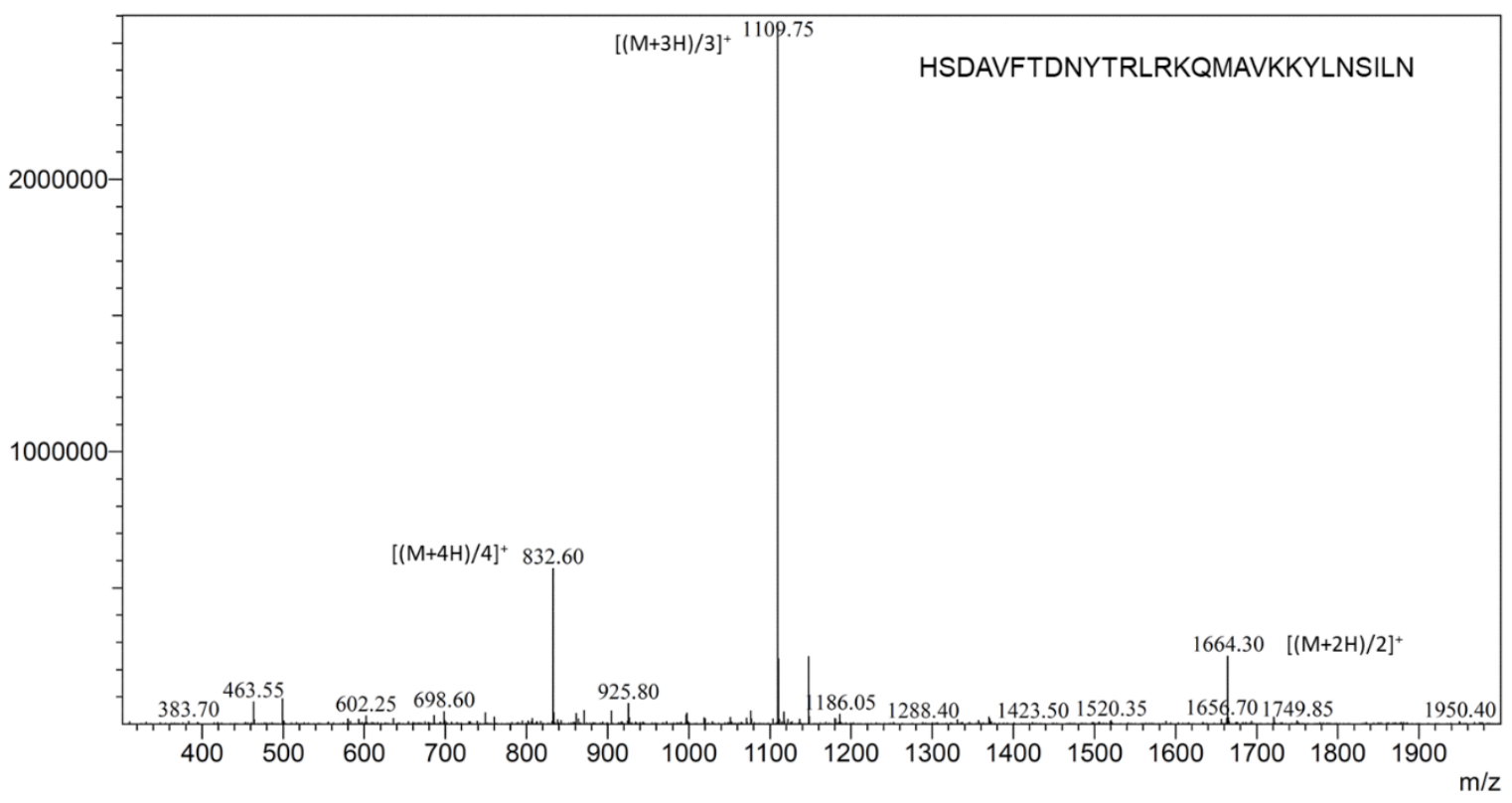

Figure S56. ESI-MS spectrum of peak at $t_{R}=11.5$ min resulted from chromatogram of entry 8 (VIP, Table 3).

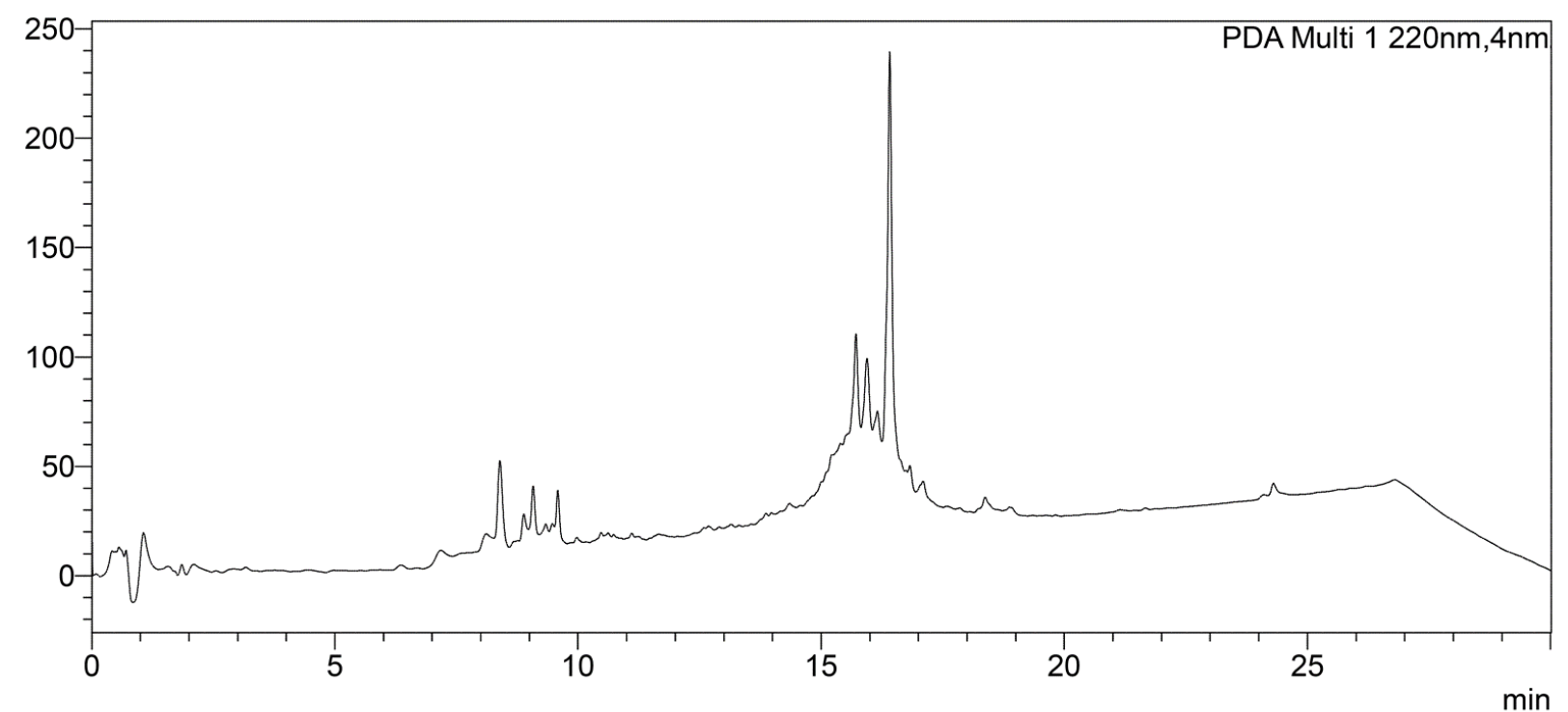

Figure S57. Chromatogram of entry 9 (GRF, Table 3) obtained by RP-HPLC (Shimadzu Prominance UFLC, SPD-M20A/DGU-20A3R/LC-20AD) on a Phenomenex Kinetex column $(\mathrm{C} 18,150 \mathrm{~mm} \times 4.6$ $\mathrm{mm}, 5 \mu \mathrm{m}, 100 \AA$ ). Purity check/Gradient $3-\mathrm{t}_{\mathrm{R}}=16.5 \mathrm{~min}$. 


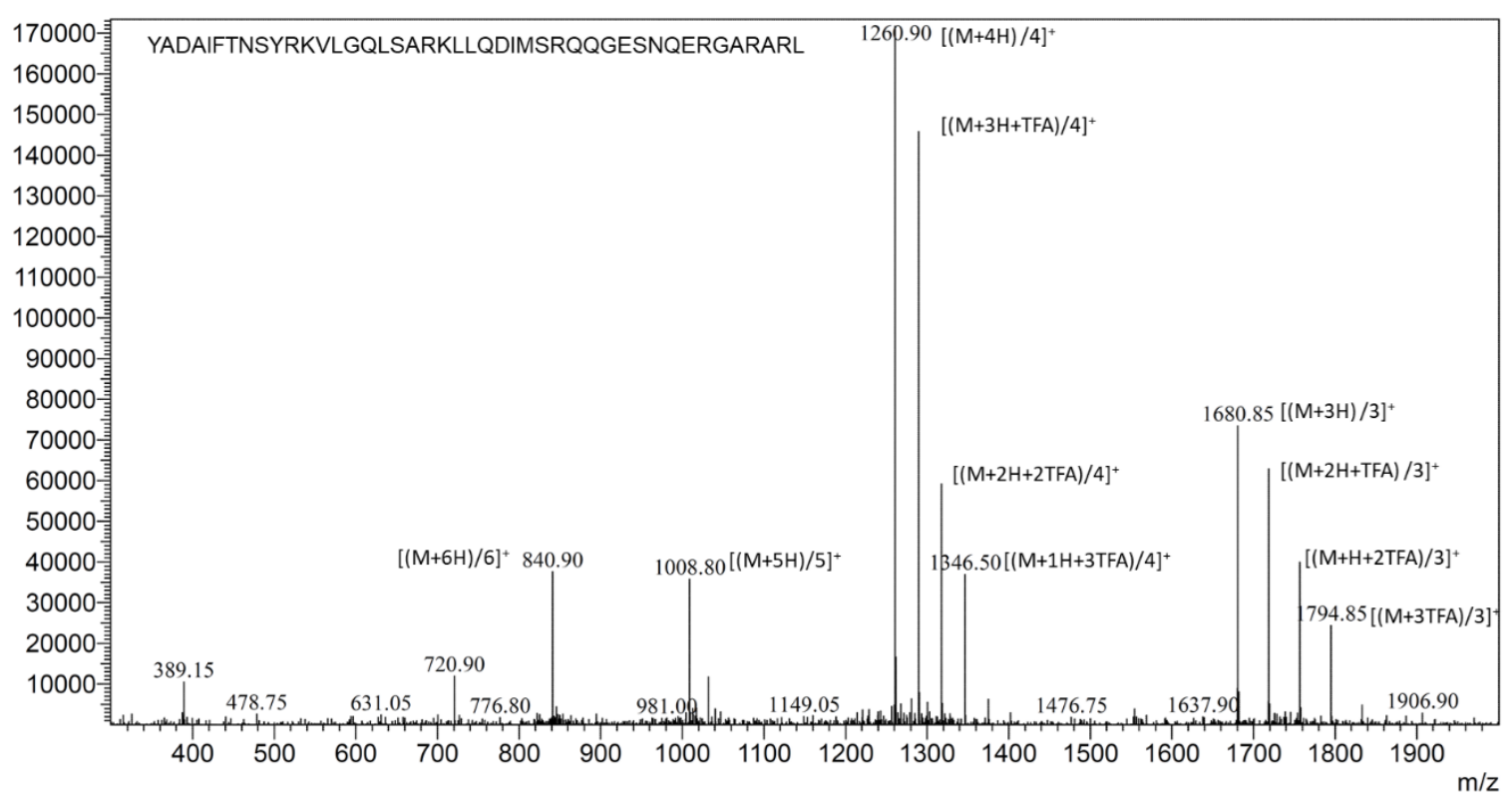

Figure S58. ESI-MS spectrum of peak at $t_{R}=16.5$ min resulted from chromatogram of entry 9 (GRF, Table 3).

\section{Difficult sequences}

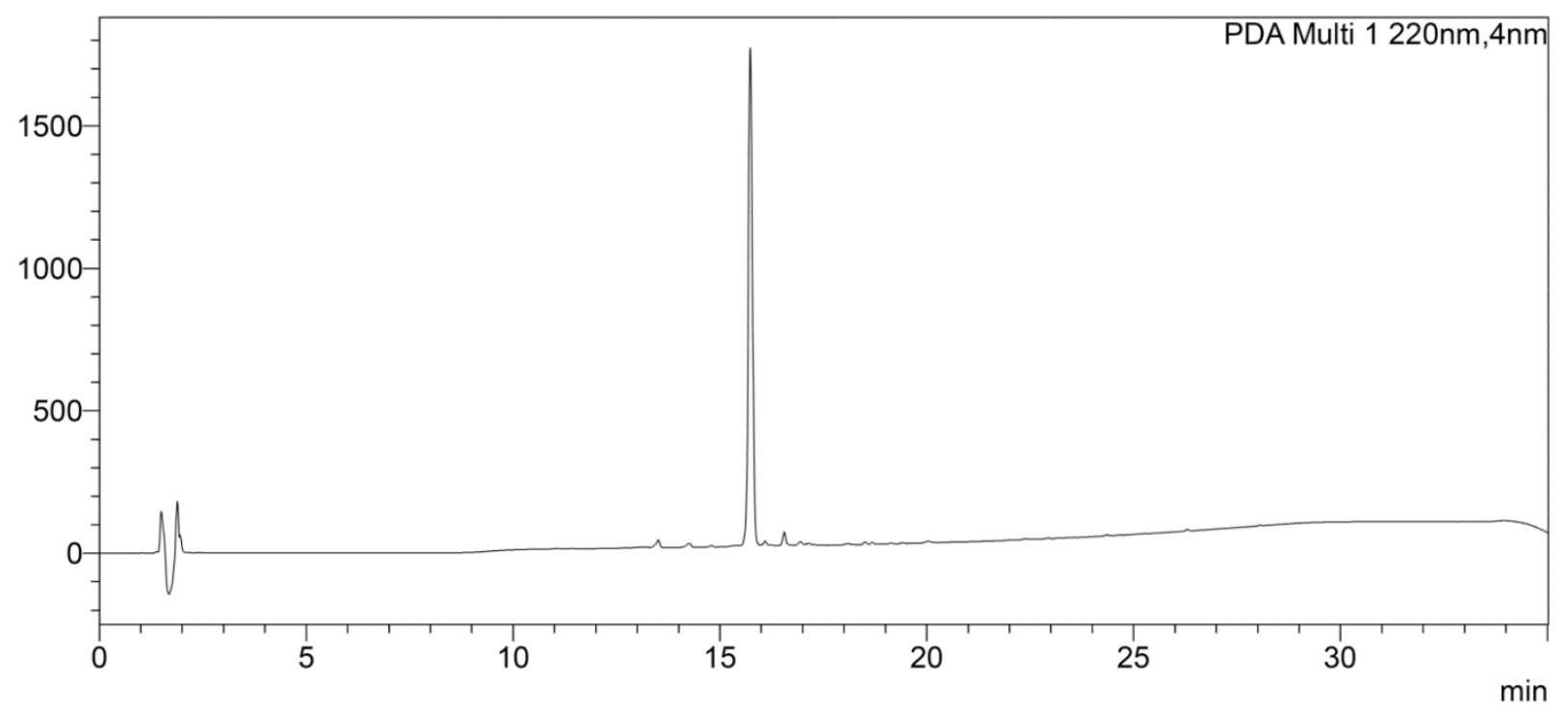

Figure S59. Representative chromatogram of entry 1a (Aib-Enk) obtained by RP-HPLC (Shimadzu Prominance UFLC, SPD-M20A/DGU-20A3R/LC-20AD) on a Phenomenex Kinetex column (C18, 150 $\mathrm{mm} \times 4.6 \mathrm{~mm}, 5 \mu \mathrm{m}, 100 \AA$ ) . Purity check/Gradient $1-\mathrm{t}_{\mathrm{R}}=15.5 \mathrm{~min}$. 


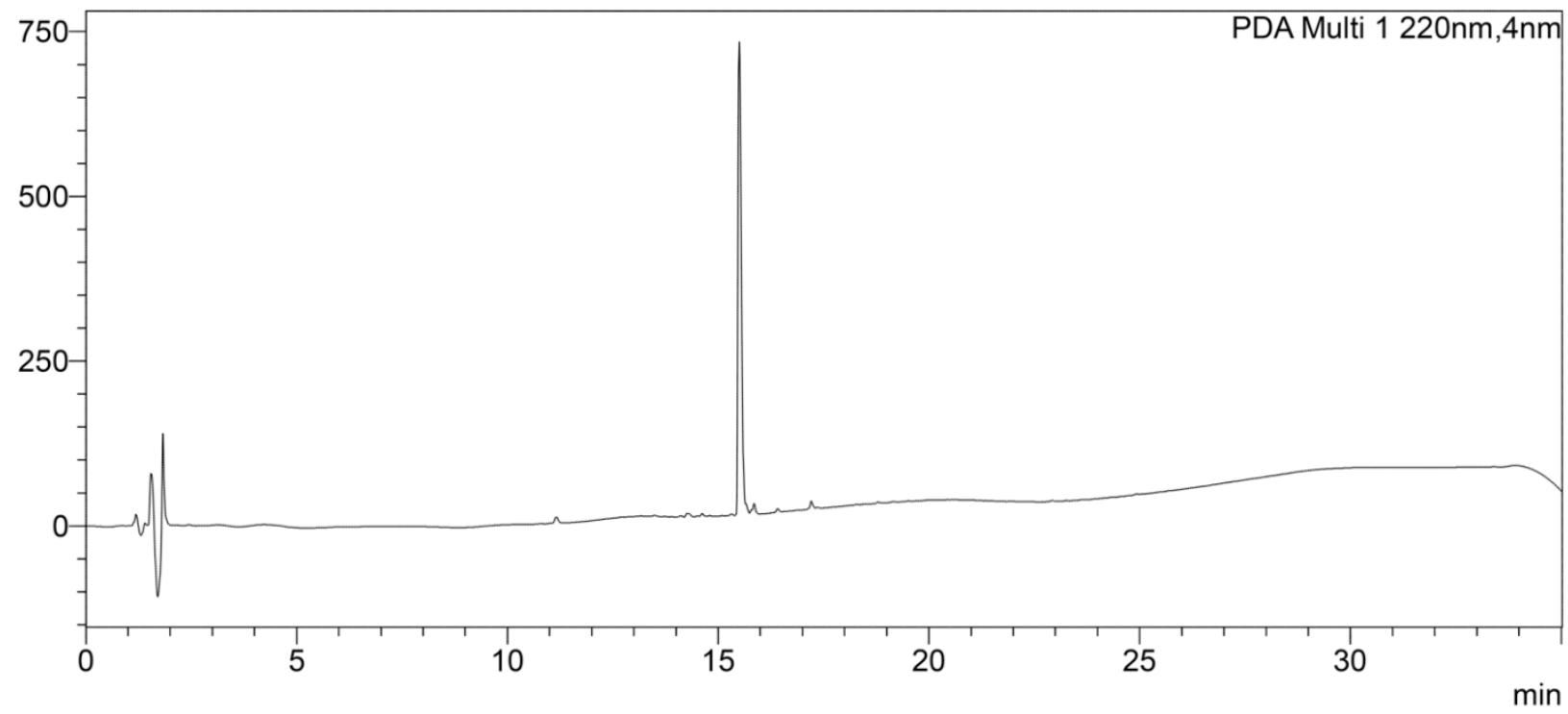

Figure S60. Representative chromatogram of entry 1b (Aib-Enk) obtained by RP-HPLC (Shimadzu Prominance UFLC, SPD-M20A/DGU-20A3R/LC-20AD) on a Phenomenex Kinetex column (C18, 150 $\mathrm{mm} \times 4.6 \mathrm{~mm}, 5 \mu \mathrm{m}, 100 \AA$ ). Purity check/Gradient $1-\mathrm{t}_{\mathrm{R}}=15.5 \mathrm{~min}$.

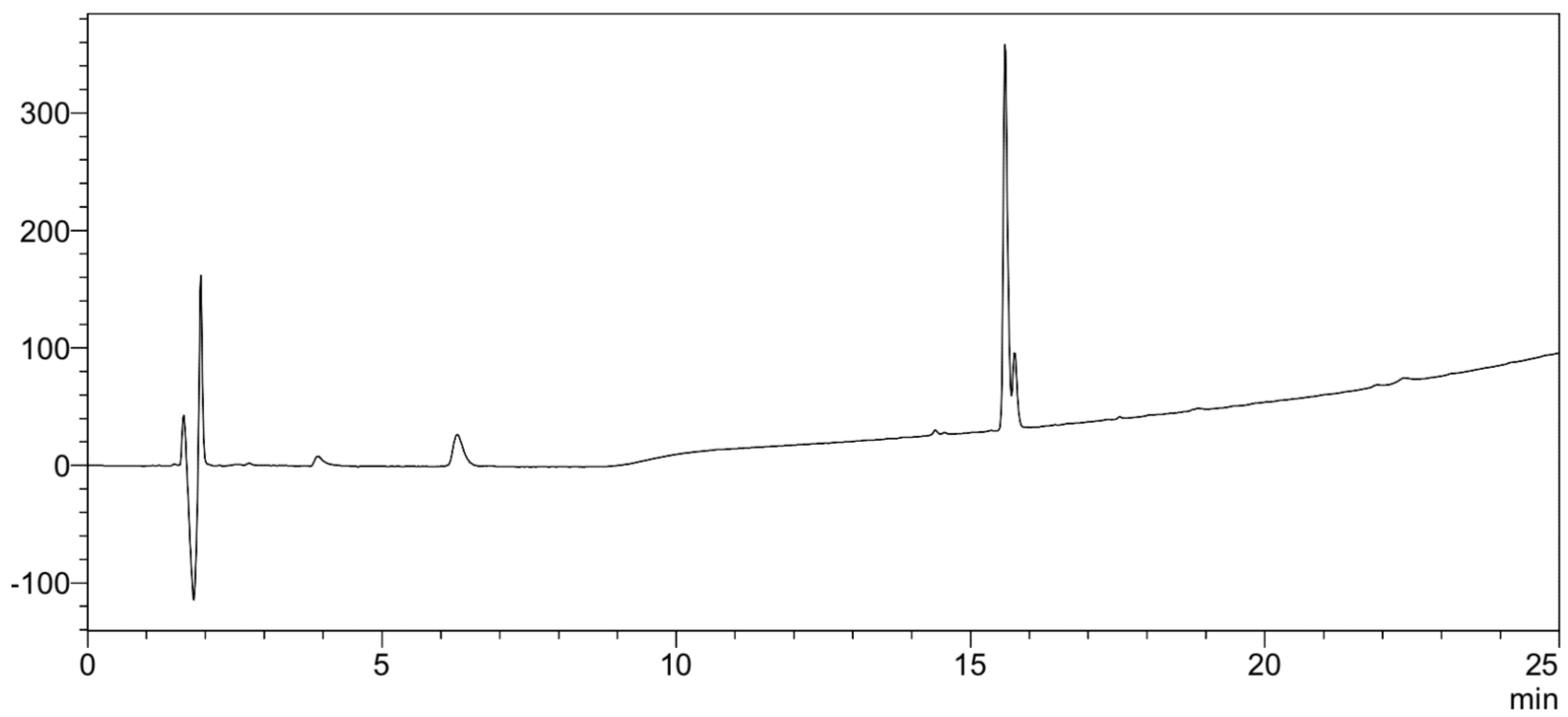

Figure S61. Representative chromatogram of entry 1c (Aib-Enk) obtained by RP-HPLC (Shimadzu Prominance UFLC, SPD-M20A/DGU-20A3R/LC-20AD) on a Phenomenex Kinetex column (C18, 150 $\mathrm{mm} \times 4.6 \mathrm{~mm}, 5 \mu \mathrm{m}, 100 \AA)$. Purity check/Gradient $1-\mathrm{t}_{\mathrm{R}}=15.5 \mathrm{~min}$. 


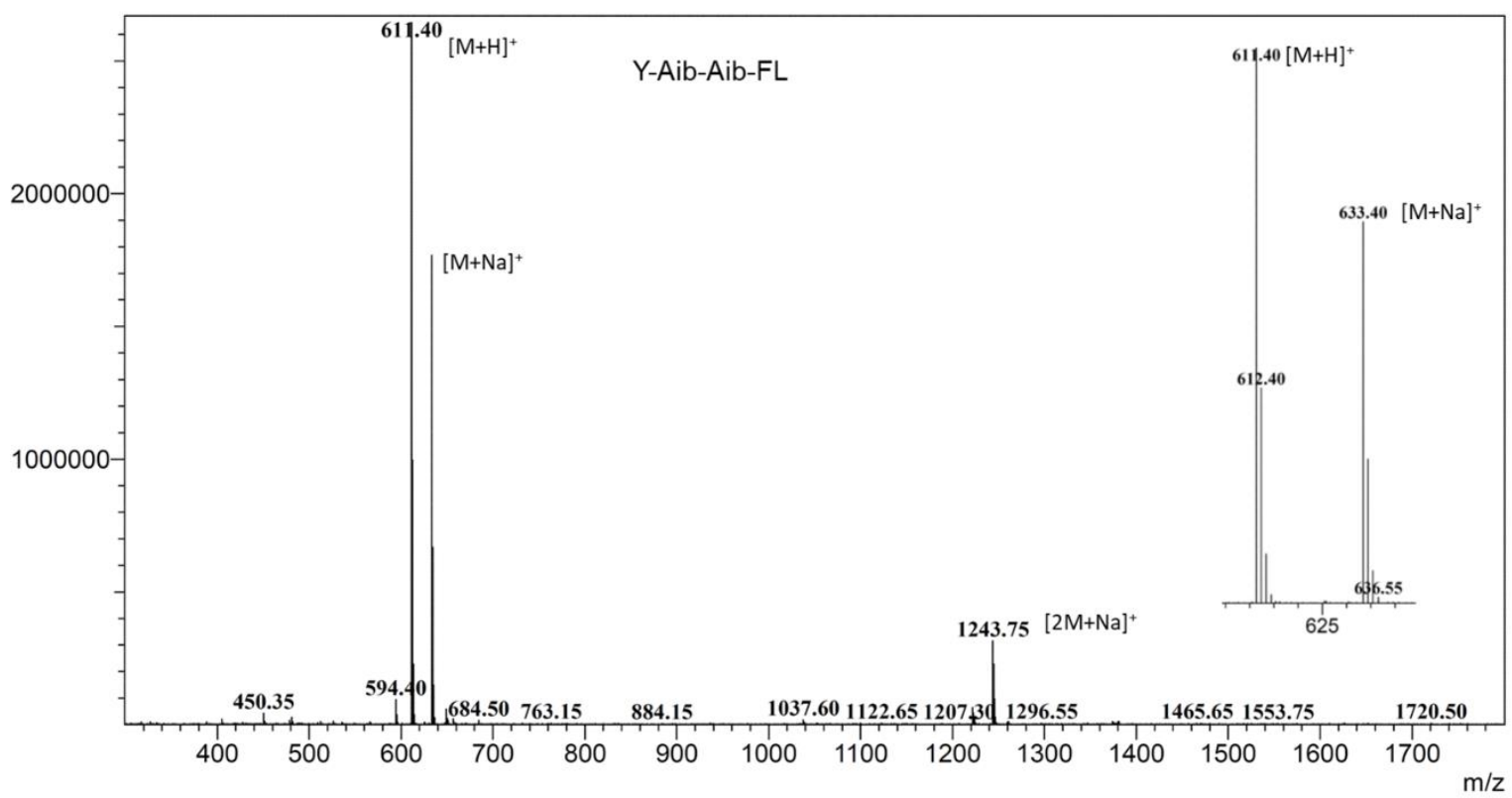

Figure S62. ESI-MS spectrum of peak at $t_{R}=15.5$ min resulted from representative chromatograms of entries 1a-c (Aib-Enk).

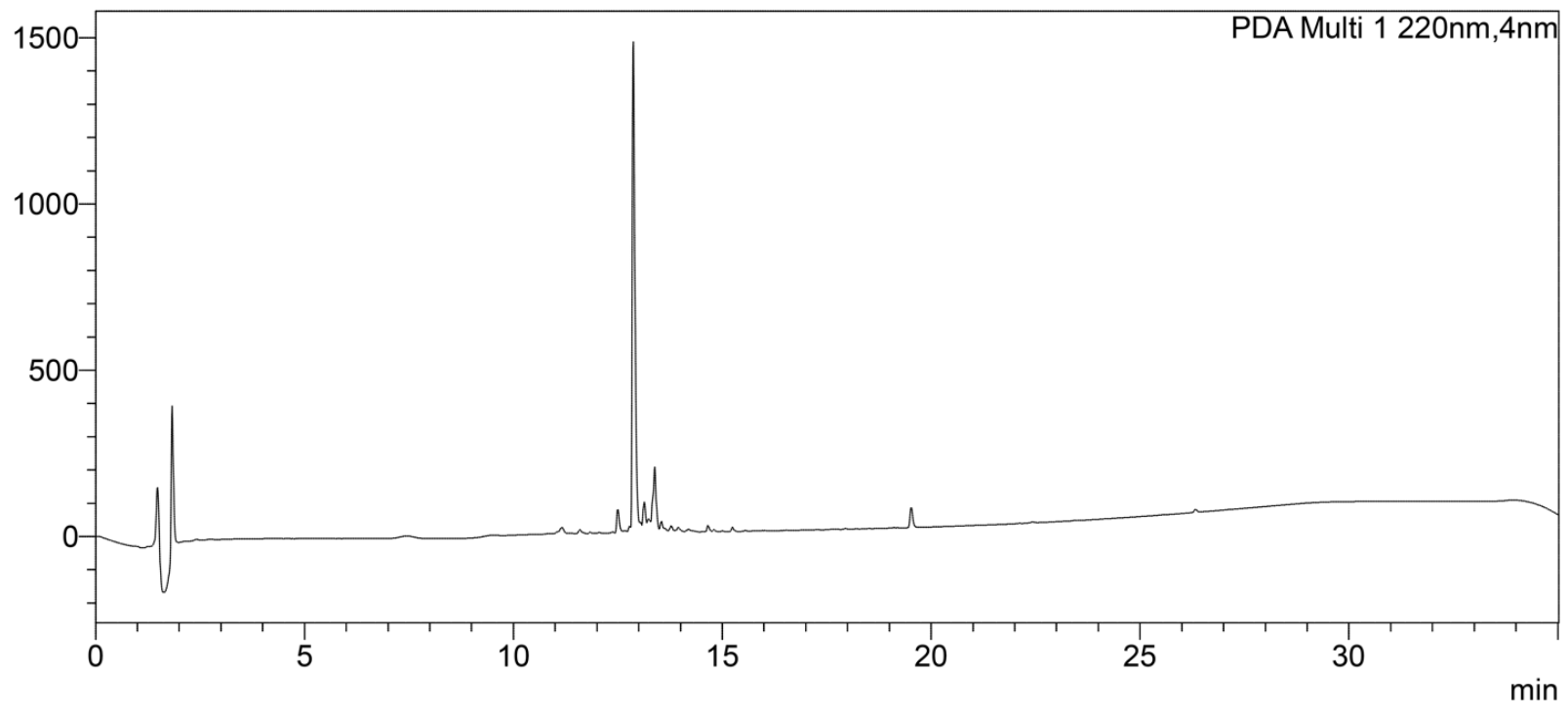

Figure S63. Representative chromatogram of entry 2a (ACP $65-74)$ obtained by RP-HPLC (Shimadzu Prominance UFLC, SPD-M20A/DGU-20A3R/LC-20AD) on a Phenomenex Kinetex column (C18, 150 $\mathrm{mm} \times 4.6 \mathrm{~mm}, 5 \mu \mathrm{m}, 100 \AA$ ). Purity check/Gradient $1-\mathrm{t}_{\mathrm{R}}=12.9 \mathrm{~min}$. 


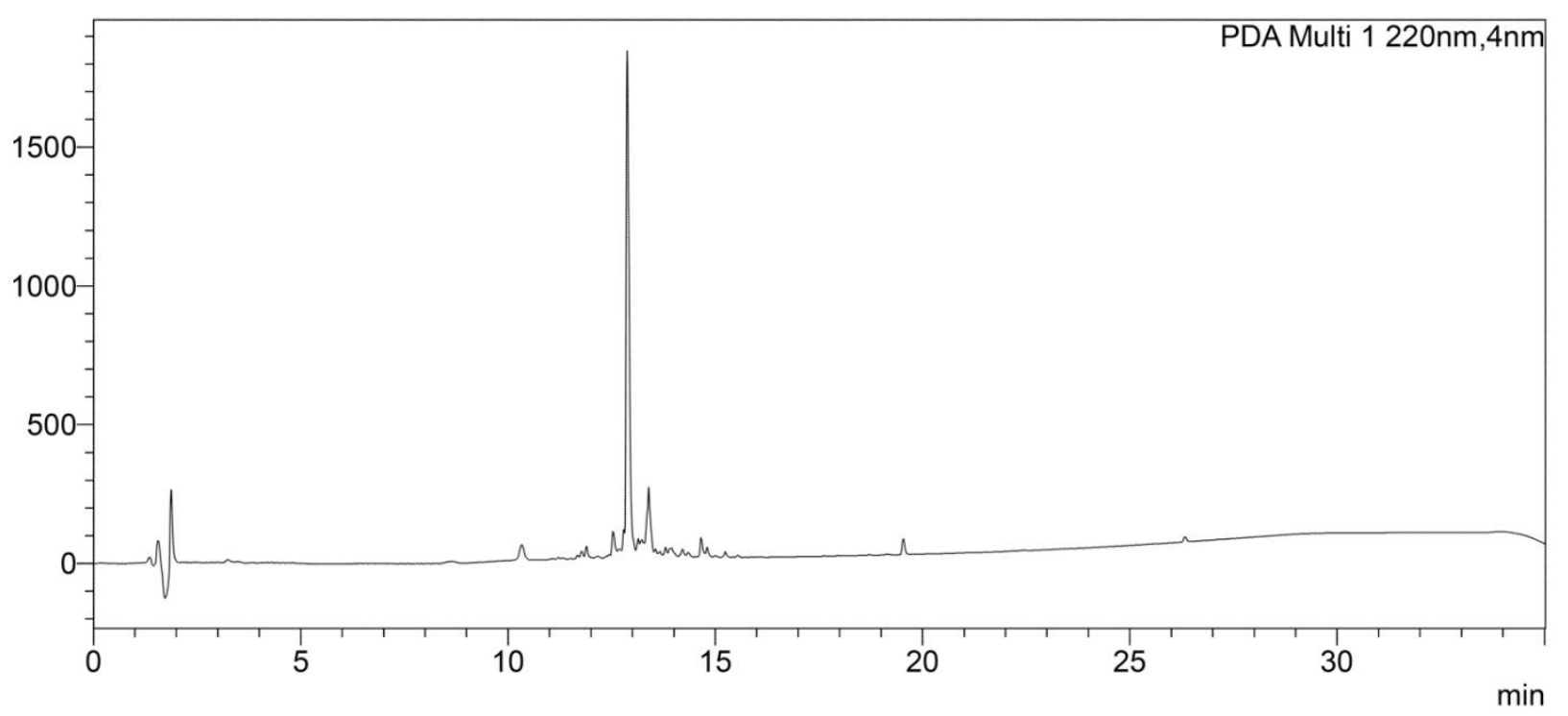

Figure S64. Representative chromatogram of entry 2b (ACP $65-74)$ obtained by RP-HPLC (Shimadzu Prominance UFLC, SPD-M20A/DGU-20A3R/LC-20AD) on a Phenomenex Kinetex column (C18, 150 $\mathrm{mm} \times 4.6 \mathrm{~mm}, 5 \mu \mathrm{m}, 100 \AA$ ). Purity check/Gradient $1-\mathrm{t}_{\mathrm{R}}=12.9 \mathrm{~min}$.

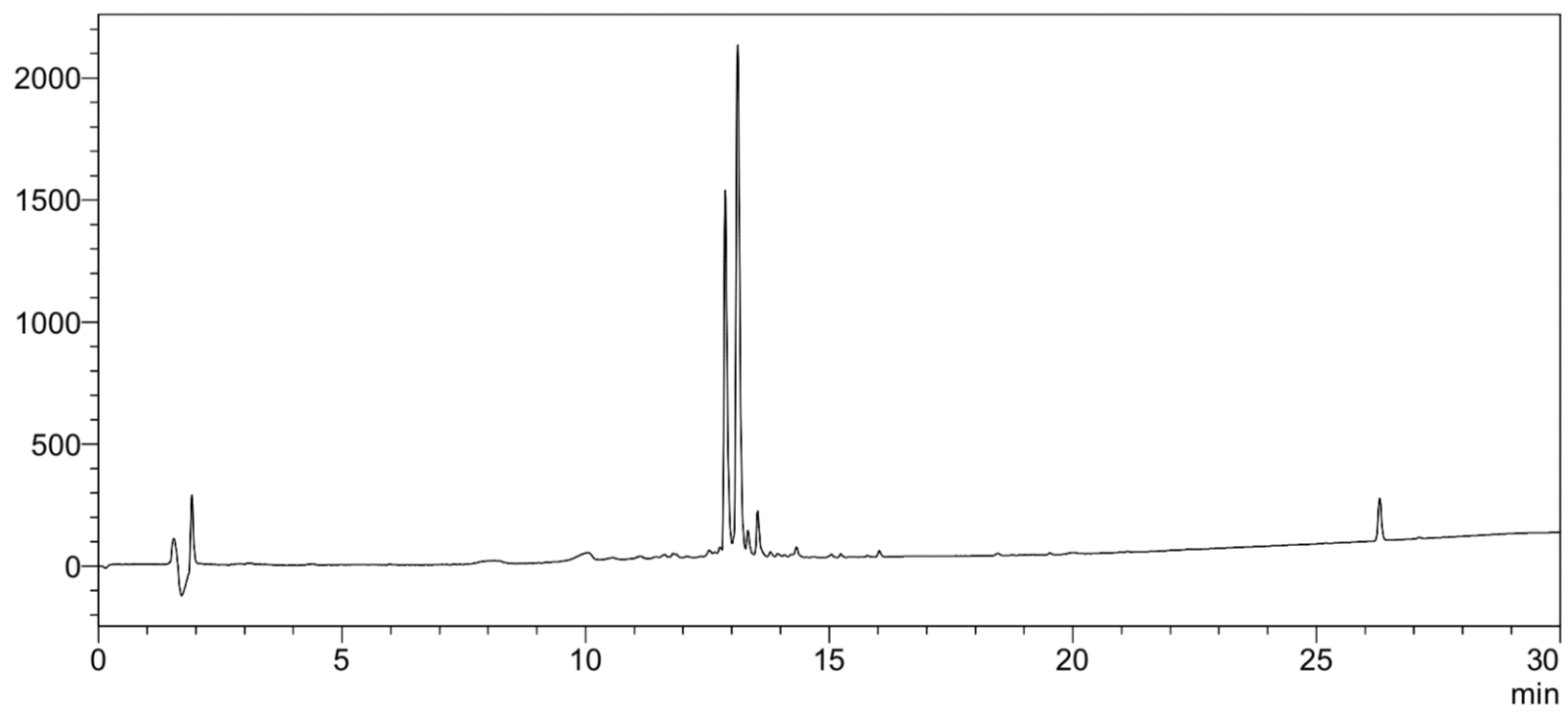

Figure S65. Representative chromatogram of entry 2c (ACP $65-74)$ obtained by RP-HPLC (Shimadzu Prominance UFLC, SPD-M20A/DGU-20A3R/LC-20AD) on a Phenomenex Kinetex column (C18, 150 $\mathrm{mm} \times 4.6 \mathrm{~mm}, 5 \mu \mathrm{m}, 100 \AA$ ) . Purity check/Gradient $1-\mathrm{t}_{\mathrm{R}}=12.9 \mathrm{~min}$. 


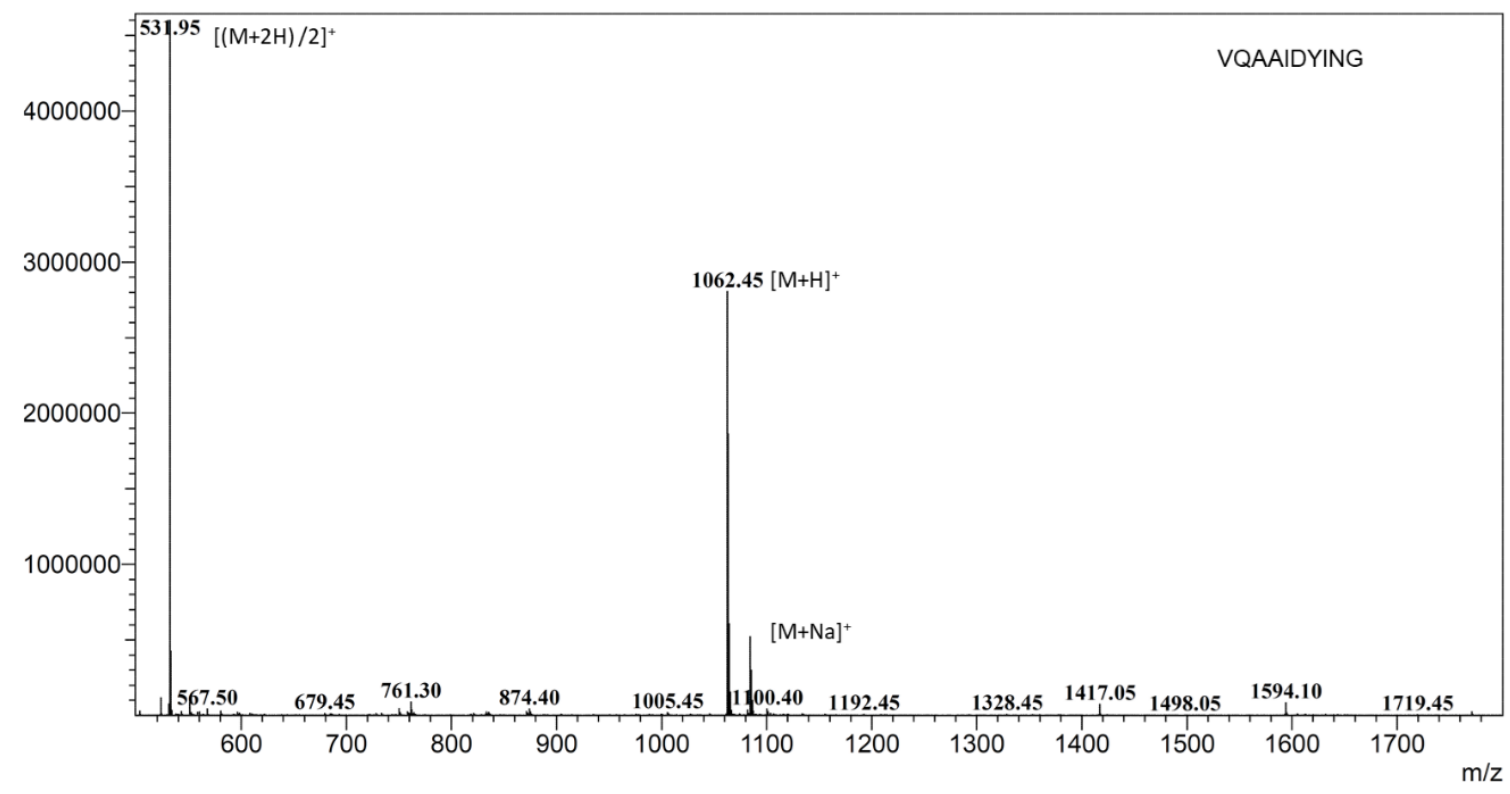

Figure S66. ESI-MS spectrum of peak at $t_{R}=12.9$ min resulted from representative chromatograms of entries 2a-c $\left(\mathrm{ACP}_{65-74}\right)$.

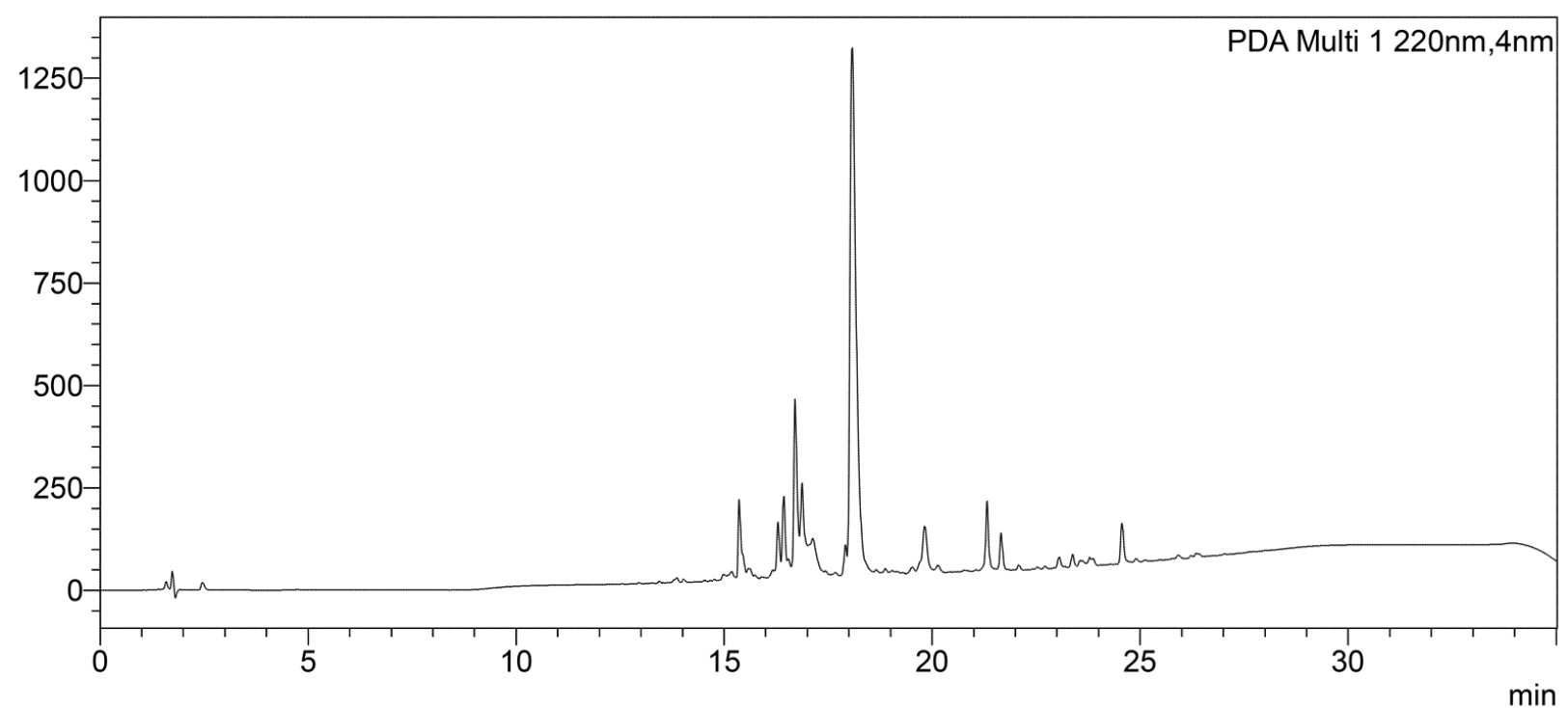

Figure S67. Representative chromatogram of entry 3a (JR 10-mer) obtained by RP-HPLC (Shimadzu Prominance UFLC, SPD-M20A/DGU-20A3R/LC-20AD) on a Phenomenex Kinetex column (C18, 150 $\mathrm{mm} \times 4.6 \mathrm{~mm}, 5 \mu \mathrm{m}, 100 \AA$ ). Purity check/Gradient $1-\mathrm{t}_{\mathrm{R}}=18.1 \mathrm{~min}$. 


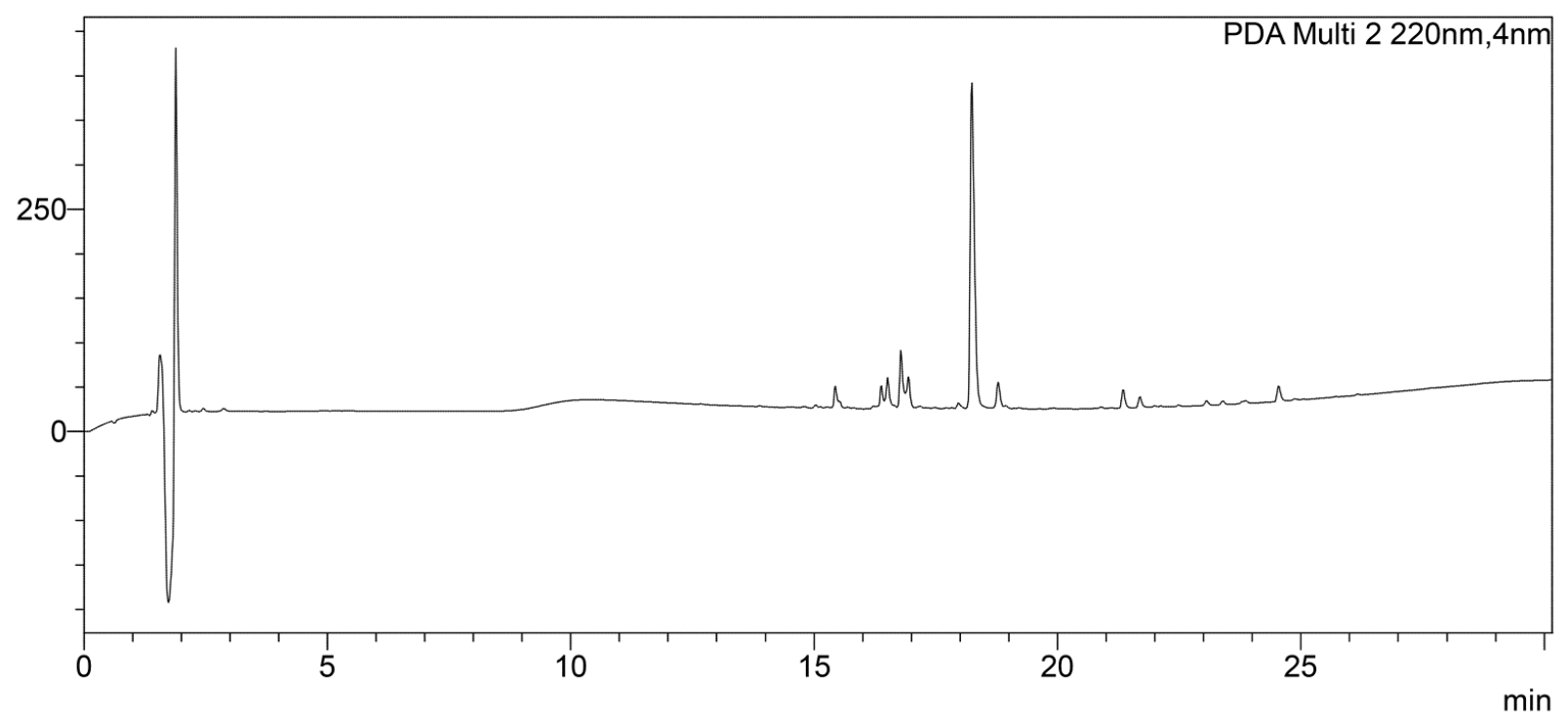

Figure S68. Representative chromatogram of entry 3b (JR 10-mer) obtained by RP-HPLC (Shimadzu Prominance UFLC, SPD-M20A/DGU-20A3R/LC-20AD) on a Phenomenex Kinetex column (C18, 150 $\mathrm{mm} \times 4.6 \mathrm{~mm}, 5 \mu \mathrm{m}, 100 \AA$ ). Purity check/Gradient $1-\mathrm{t}_{\mathrm{R}}=18.1 \mathrm{~min}$.

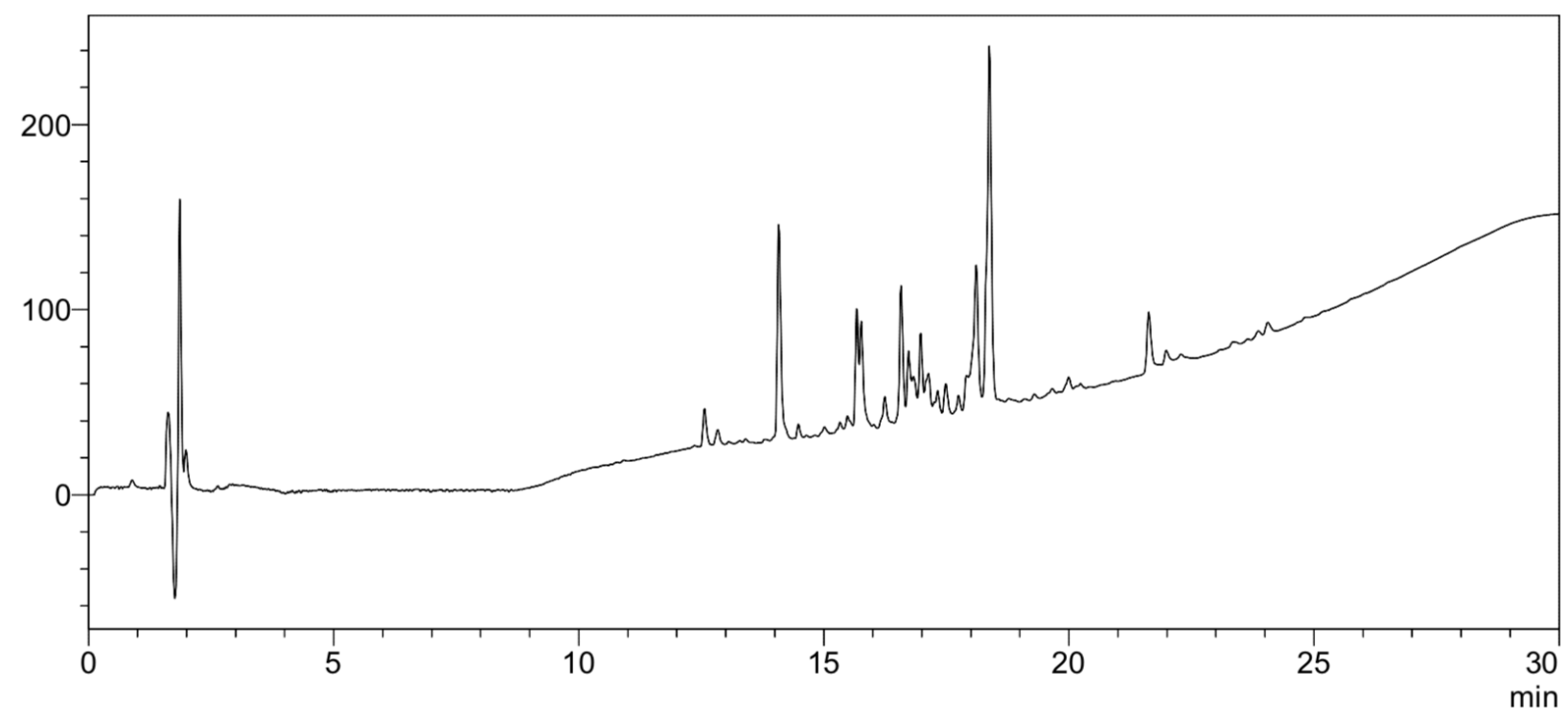

Figure S69. Representative chromatogram of entry 3c (JR 10-mer) obtained by RP-HPLC (Shimadzu Prominance UFLC, SPD-M20A/DGU-20A3R/LC-20AD) on a Phenomenex Kinetex column (C18, 150 $\mathrm{mm} \times 4.6 \mathrm{~mm}, 5 \mu \mathrm{m}, 100 \AA$ ) $)$ Purity check/Gradient $1-\mathrm{t}_{\mathrm{R}}=18.1 \mathrm{~min}$. 


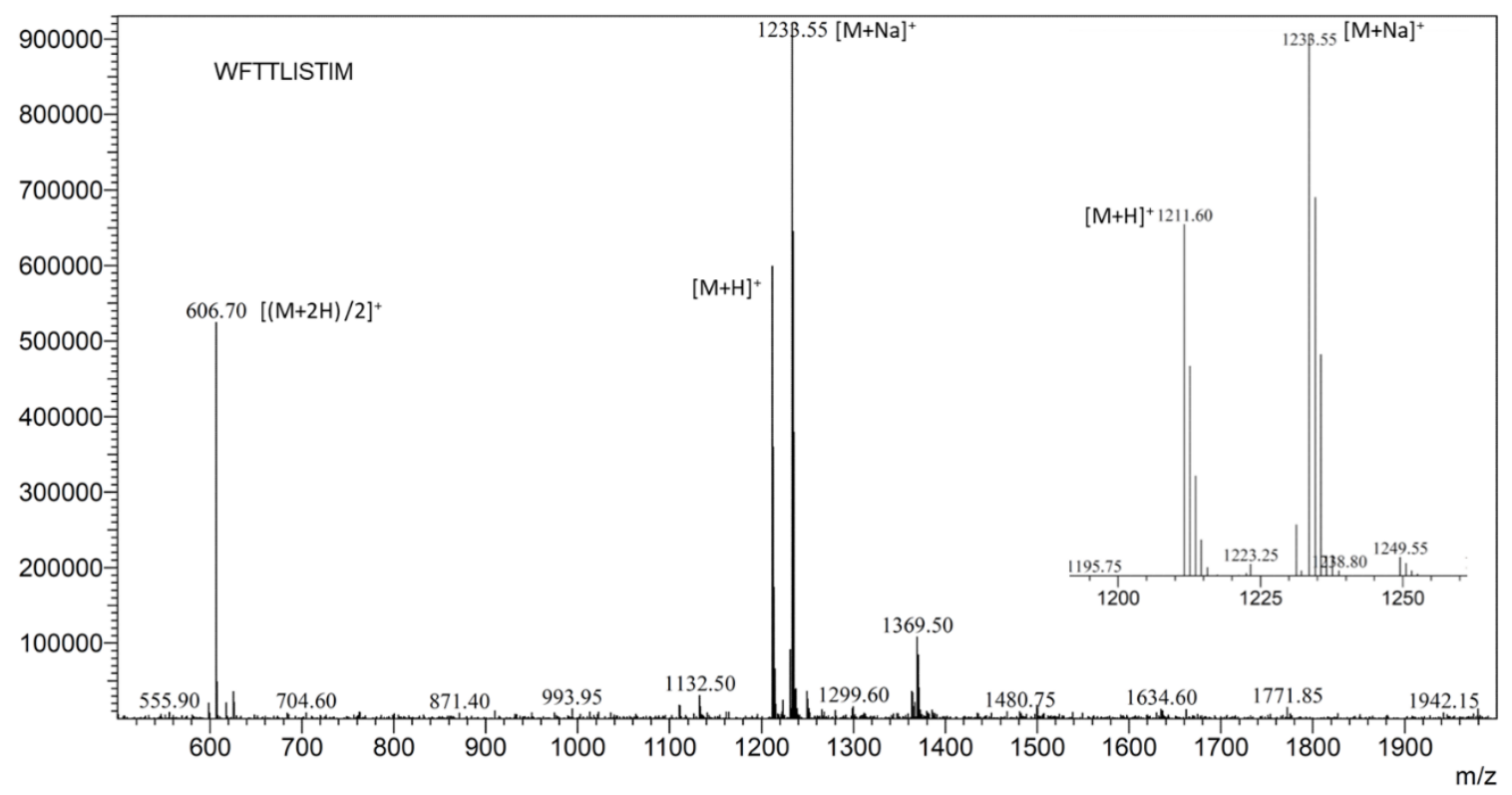

Figure S70. ESI-MS spectrum of peak at $t_{R}=18.1 \mathrm{~min}$ resulted from representative chromatograms of 3a-c (JR 10-mer).

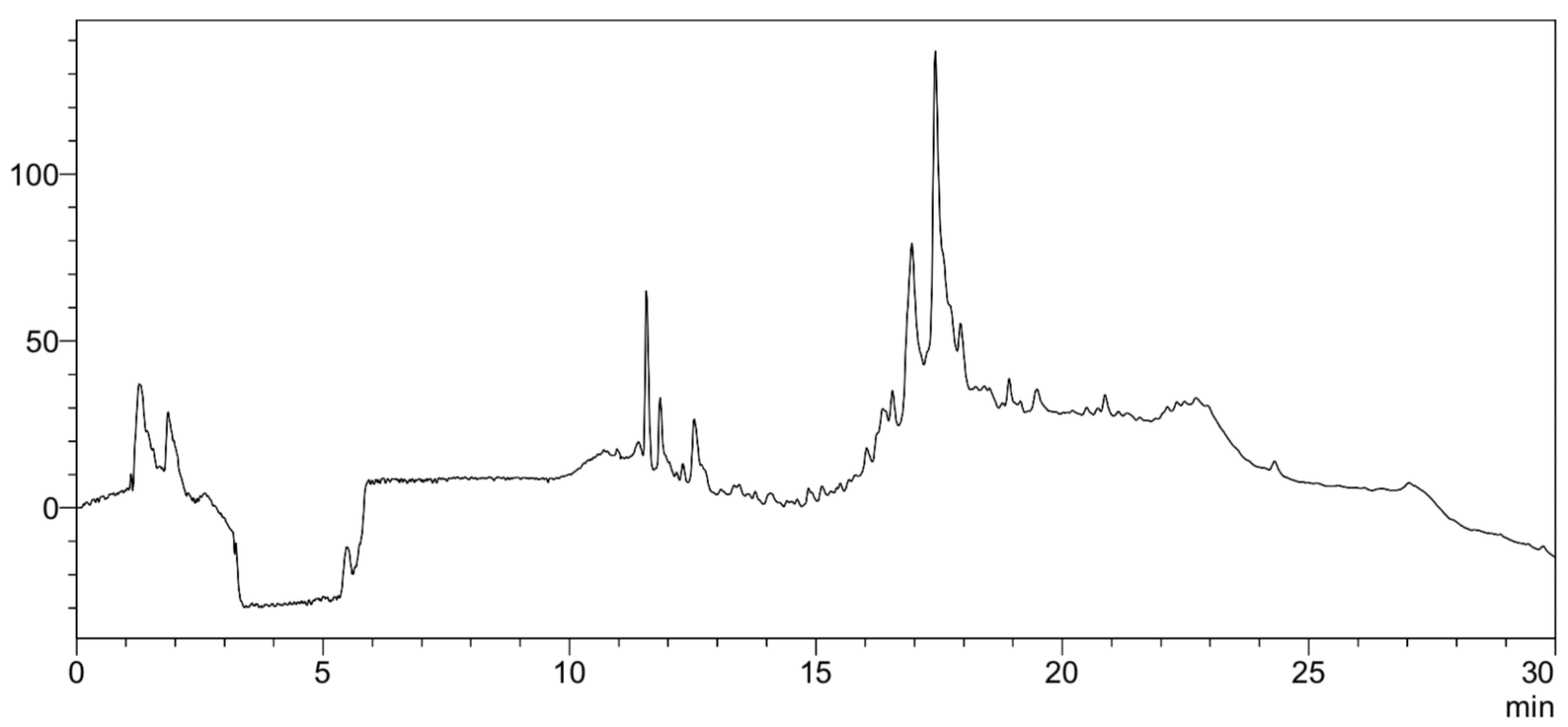

Figure S71. Representative chromatogram of entry $4 \mathbf{a}(\mathrm{A} \beta$ 1-42) obtained by RP-HPLC (Shimadzu Prominance UFLC, SPD-M20A/DGU-20A3R/LC-20AD) on a Phenomenex Kinetex column (C18, 150 $\mathrm{mm} \times 4.6 \mathrm{~mm}, 5 \mu \mathrm{m}, 100 \AA$ ). Purity check/Gradient $4-\mathrm{t}_{\mathrm{R}}=17.3 \mathrm{~min}$. 


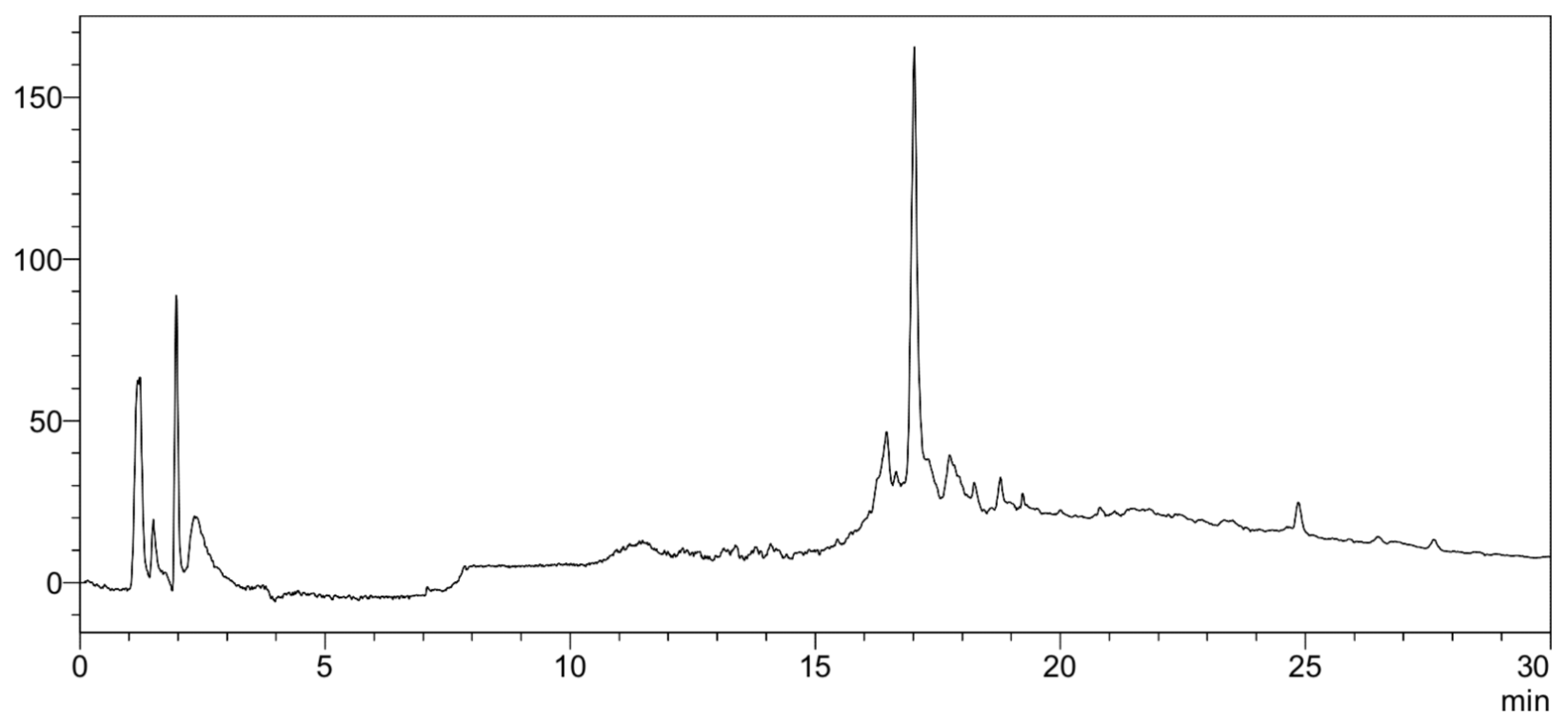

Figure S72. Representative chromatogram of entry $\mathbf{4 b}$ (A $\beta$ 1-42) obtained by RP-HPLC (Shimadzu Prominance UFLC, SPD-M20A/DGU-20A3R/LC-20AD) on a Phenomenex Kinetex column (C18, 150 $\mathrm{mm} \times 4.6 \mathrm{~mm}, 5 \mu \mathrm{m}, 100 \AA$ ). Purity check/Gradient $4-\mathrm{t}_{\mathrm{R}}=17.3 \mathrm{~min}$.

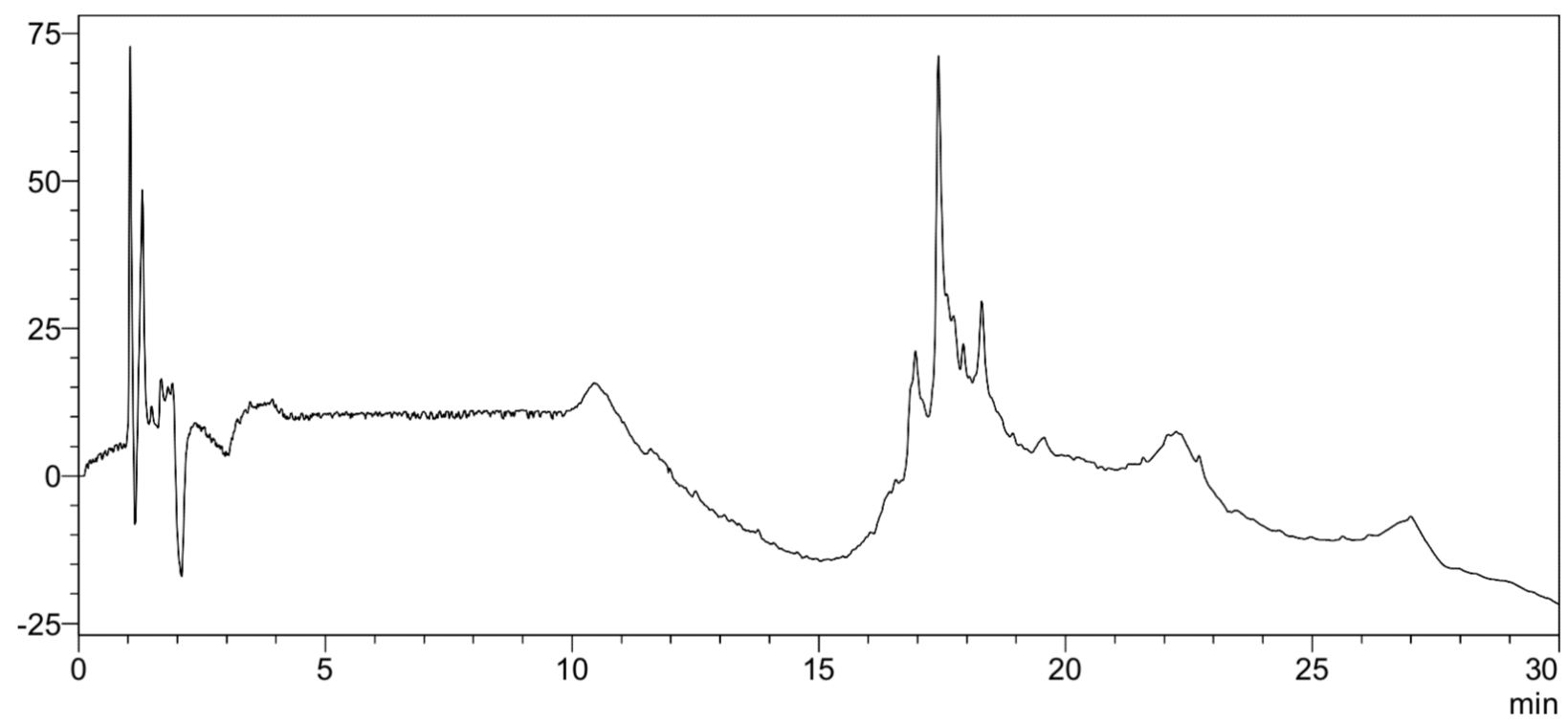

Figure S73. Representative chromatogram of entry 4c (A $\beta$ 1-42) obtained by RP-HPLC (Shimadzu Prominance UFLC, SPD-M20A/DGU-20A3R/LC-20AD) on a Phenomenex Kinetex column (C18, 150 $\mathrm{mm} \times 4.6 \mathrm{~mm}, 5 \mu \mathrm{m}, 100 \AA$ ) . Purity check/Gradient $4-\mathrm{t}_{\mathrm{R}}=17.3 \mathrm{~min}$. 


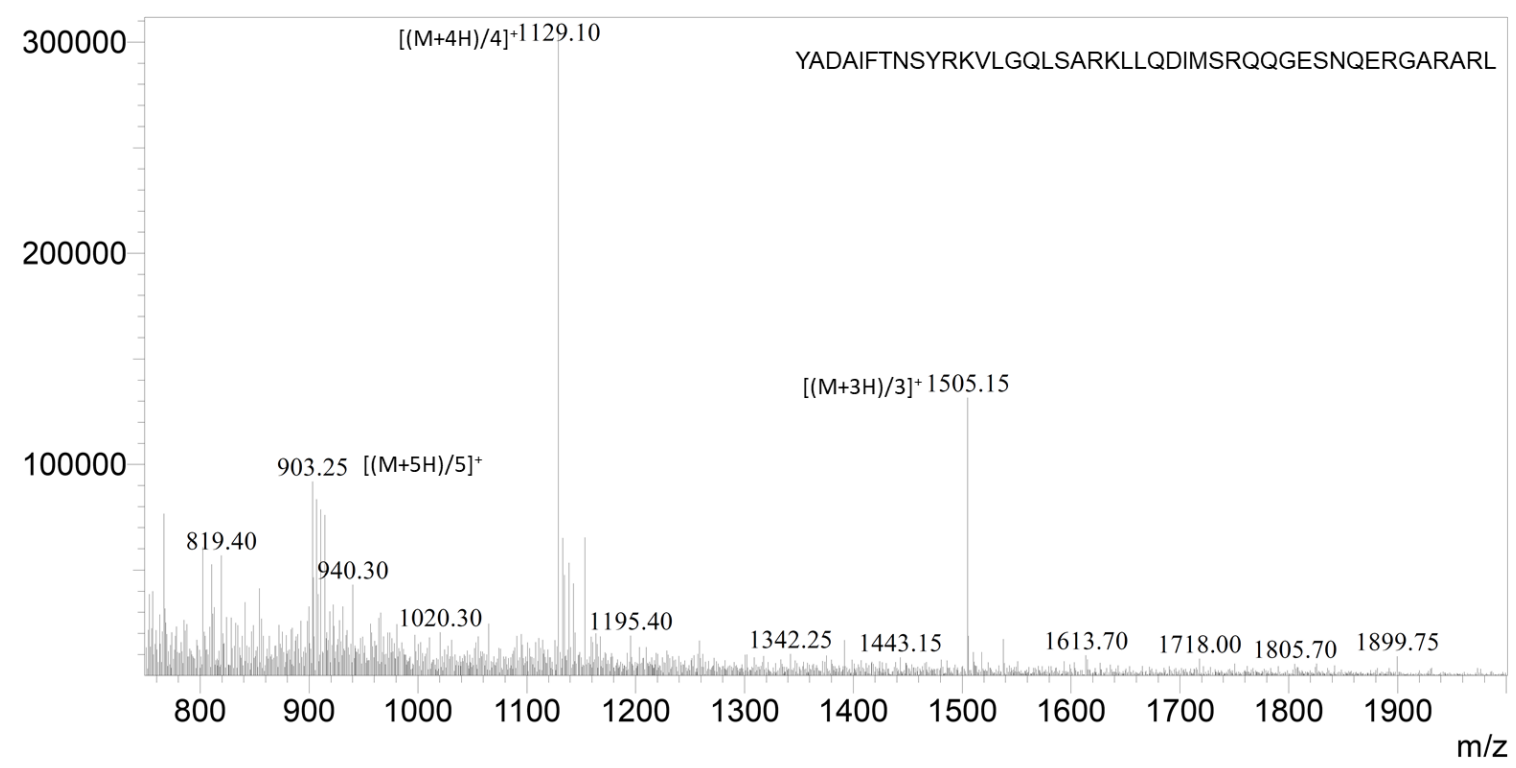

Figure S74. ESI-MS spectrum of peak at $t_{R}=17.3$ min resulted from representative chromatograms of entries 4a-c (A $\beta$ 1-42).

\section{References}

(1) Kaiser, E.; Colescott, R. L.; Bossinger, C. D.; Cook, P. I. Color test for detection of free terminal amino groups in the solid-phase synthesis of peptides. Anal. Biochem. 1970, 34, 595-598.

(2) Hancock, W. S. and Battersby, J. E. A new micro-test for the detection of incomplete coupling reactions in solid-phase peptide synthesis using 2,4,6- trinitrobenzenesulphonic acid. Anal. Biochem. 1976, 71, 260-264.

(3) Vojkovsky, T. Detection of secondary amines on solid phase. Pept Res. 1995, 8, 236-237.

(4) Mason, T. J. and Lorimer J. P. in Applied sonochemistry. the uses of power ultrasound in chemistry and processing; Wiley-VCH, Weinheim, 2002.

(5) Subirã-Funosas, R.; Prohens, R.; Barbas, R.; El-Faham, A.; Albericio, F. Oxyma: an efficient additive for peptide synthesis to replace the benzotriazole-based HOBt and HOAt with a lower risk of explosion. Chem. Eur. J. 2009, 15, 9394-9403. 